\title{
A Master Autoantigen-ome Links Alternative Splicing, Female Predilection, and COVID-19 to Autoimmune Diseases
}

Julia Y. Wang ${ }^{1 *}$, Michael W. Roehrl ${ }^{1}$, Victor B. Roehrl ${ }^{1}$, and Michael H. Roehrl ${ }^{2 *}$

${ }^{1}$ Curandis, New York, USA

${ }^{2}$ Department of Pathology, Memorial Sloan Kettering Cancer Center, New York, USA

\footnotetext{
*Correspondence: julia@curandis.com or roehrlm@mskcc.org
} 


\begin{abstract}
Chronic and debilitating autoimmune sequelae pose a grave concern for the post-COVID-19 pandemic era. Based on our discovery that the glycosaminoglycan dermatan sulfate (DS) displays peculiar affinity to apoptotic cells and autoantigens (autoAgs) and that DS-autoAg complexes cooperatively stimulate autoreactive B1 cell responses, we compiled a database of 751 candidate autoAgs from six human cell types. At least 657 of these have been found to be affected by SARS-CoV-2 infection based on currently available multi-omic COVID data, and at least 400 are confirmed targets of autoantibodies in a wide array of autoimmune diseases and cancer. The autoantigen-ome is significantly associated with various processes in viral infections, such as translation, protein processing, and vesicle transport. Interestingly, the coding genes of autoAgs predominantly contain multiple exons with many possible alternative splicing variants, short transcripts, and short UTR lengths. These observations and the finding that numerous autoAgs involved in RNA-splicing showed altered expression in viral infections suggest that viruses exploit alternative splicing to reprogram host cell machinery to ensure viral replication and survival. While each cell type gives rise to a unique pool of autoAgs, 39 common autoAgs associated with cell stress and apoptosis were identified from all six cell types, with several being known markers of systemic autoimmune diseases. In particular, the common autoAg UBA1 that catalyzes the first step in ubiquitination is encoded by an X-chromosome escape gene. Given its essential function in apoptotic cell clearance and that X-inactivation escape tends to increase with aging, UBA1 dysfunction can therefore predispose aging women to autoimmune disorders. In summary, we propose a model of how viral infections lead to extensive molecular alterations and host cell death, autoimmune responses facilitated by autoAg-DS complexes, and ultimately autoimmune diseases. Overall, this master autoantigen-ome provides a molecular guide for investigating the myriad of autoimmune sequalae to COVID-19 and clues to the rare but reported adverse effects of the currently available COVID vaccines.
\end{abstract}




\section{Introduction}

Autoimmune disorders are an important feature of the disease manifestations of COVID-19 and longCOVID syndromes. Based on the insights we gained from numerous COVID-related autoantigens (autoAgs) and their associated cellular process and pathways [1-5], we propose a model to explain how viral infections in general and SARS-CoV-2 in particular can lead to a wide array of autoimmune diseases (Figure 1). We illustrate how viral infections lead to extensive molecular alterations in the host cell, host cell death and tissue injury, autoimmune reactions, and the eventual development of autoimmune diseases.

During infections, opportunistic viruses have to hijack the host cell machinery in order to transcribe and translate the viral genes, synthesize viral proteins with correct polypeptide folding and post-translational modifications, and assemble viral particles. At the same time, viruses have to manipulate the host's immune defense to avoid elimination. This intricate host-virus symbiosis is accomplished by extensive alterations of host molecules and reprogramming of host molecular networks. The infected host cells undergo extreme stress and ultimately die, which releases altered molecules (i.e., potential autoAgs) that the immune system may recognize as non-self. In response, the host also synthesizes a cascade of molecules such as dermatan sulfate (DS) to facilitate wound healing and dead cell clearance.

We have discovered previously that DS possesses peculiar affinity for apoptotic cells and their released autoAgs [6-9]. DS, a major component of the extracellular matrix and connective tissue, is increasingly expressed during tissue injury and accumulates in wound areas $[1,10]$. Because of their affinity, DS and autoAgs form macromolecular complexes which cooperatively activate autoreactive B1 cells. AutoAg-DS complexes may activate B1 cells via a dual binding mode, i.e., with autoAg binding to the variable region of the B1 cell's autoBCR and DS binding to the heavy chain of the autoBCR. Upon entering B1 cells, DS may regulate immunoglobulin (Ig) production by engaging the Ig-processing complex in the endoplasmic reticulum and the transcription factor GTF2I necessary for Ig gene expression [8, 9]. AutoAg-DS affinity therefore defines a unifying biochemical and immunological property of autoAgs: any self-molecule possessing DS-affinity has a high propensity to become autoantigenic, and this has led to the identification of numerous autoAgs [7, 11-13].

To gain a better understanding of autoimmune sequelae due to COVID-19, we present a master autoantigen atlas of over 750 potential autoAgs identified from six human cell types $[1,2,4,5,7,11]$. These autoAgs show significant correlation with pathways and processes that are crucial in viral infection and mRNA vaccine action, reveal common autoAgs associated with apoptosis and cell stress which may serve as markers for systemic autoimmune diseases, and provide a detailed molecular map for understanding and for investigating diverse autoimmune sequalae of COVID-19 and potential rare sideeffects to viral vector- and mRNA-based vaccines. For the first time, we reveal intriguing features of autoAgs and their coding genes. Furthermore, we discuss how UBA1 (or UBE1, ubiquitin-like modifieractivating enzyme 1), an autoAg found overexpressed in SARS-CoV-2 infection, may predispose aging females to autoimmune disorders. 


\section{Results and Discussion}

\section{The master autoantigen-ome}

To understand the diversity of autoimmune diseases, we were curious to know how many autoAgs possibly exist. A total of 751 potential autoAgs were identified (Table 1) when we combined all DS-affinity autoAgs profiled from six human cell lines, namely, HFL1 fetal lung fibroblasts, HEp2 fibroblasts, A549 lung epithelial cells, HS-Sultan and Wil2-NS B-lymphoblasts, and Jurkat T-lymphoblasts. Extensive literature searches confirmed that at least 400 of these proteins $(53.3 \%)$ have been reported as targets of autoantibodies in a wide variety of autoimmune diseases and cancer (see autoAg confirmation references in Table 1). The majority of unconfirmed or putative autoAgs are isoforms of or structurally similar to reported autoAgs and are yet-to-confirmed autoAgs. For example, 56 ribosomal proteins were identified by DS-affinity, but only 22 are thus far confirmed autoAgs; but given their structural similarity and shared epitopes, it is likely that most if not all of the 56 ribosomal proteins are likely true autoAgs awaiting further confirmation.

The master autoantigen-ome contains clusters of protein families, including 56 ribosomal proteins, 27 proteasome subunits, 19 heterogeneous ribonucleoproteins, 17 splicing factors, 17 ATP-dependent RNA helicase subunits, 16 eukaryotic translation initiation factors, 16 histones, 16 aminoacyl-tRNA synthases, 12 heat shock proteins, 9 elongation factors, 9 small nuclear ribonucleoproteins, 8 T-complex protein 1 subunits, and 7 14-3-3 proteins. In addition, there are multiple isoforms of numerous proteins, such as actin, tropomyosin, myosin, collagen, tubulin, and annexin.

The 751 confirmed and putative autoAgs are highly connected and have significantly more interactions than what would be expected for a random set of proteins of similar size drawn from the genome (exhibiting 6,936 interactions vs. 3,596 expected with the highest confidence level cutoff; enrichment $p$ value $<1.0 \mathrm{e}-16$ ) as per protein-protein interaction analysis in STRING [14] (Fig. 2). The 400 confirmed autoAgs also form a similar, strong interacting network (exhibiting 2,758 interactions vs. 1,269 expected; enrichment $p$ value $<10 \mathrm{e}-16$ ) (Fig. 3). The tight connections within the autoAg network suggest that these proteins are biologically connected, and given that they are all identified by DS-affinity, the autoAg protein networks offer a glimpse of the biological roles and functions of DS that await further investigation.

The 751-protein master autoantigen-ome is significantly associated with many biological processes and pathways, most notably translation, RNA processing, RNA splicing, protein folding, vesicle-mediated transport, chromosome organization, regulation of cell death, and apoptosis (Figs. 2 and 4). The 400 confirmed autoAgs are similarly significantly associated with the same processes and pathways (Fig. 3). In addition, these proteins are associated with numerous other processes, e.g., mRNA metabolic process, peptide metabolic process, establishment of localization in the cell, intracellular transport, interspecies interaction between organisms, viral process (infection and virulence), symbiotic process, and response to stress (Figs. 2-4). Hierarchical clustering [15] of the top 50 enriched Gene Ontology Biological Processes reveals RNA processing, particularly RNA splicing, to be the most noticeable (Fig. 4). 


\section{The COVID-19 autoantigen-ome}

To find out how many autoAgs in the autoantigen-ome are potentially affected by SARS-CoV-2 infection, we looked for them in currently available multi-omic COVID data compiled by Coronascape [16-37]. Remarkably, 657 (87.5\%) proteins of the 751-member master autoantigen-ome are found to be affected in SARS-CoV-2 infection (Table 1 and Supplemental Table 1). Among them, 109 proteins were found upregulated only, 176 were found down-regulated only, and 343 were found both up- and down-regulated at protein and/or RNA levels in virally infected cells or COVID-19 patients (Table 1 and Fig. 6). In addition, 191 potential autoAgs were found in the interactomes of different SARS-CoV-2 viral component proteins, meaning that they may directly or indirectly interact with the virus.

The 657-member COVID autoantigen-ome is also a highly interacting protein network (Fig. 5). Not surprisingly, these proteins are significantly associated with processes that are crucial in viral infection, e.g., RNA processing, mRNA metabolic process, regulation of mRNA stability, translation, peptide biosynthetic process, protein folding, intracellular transport, vesicle-mediated transport, regulated exocytosis, symbiont process, and interspecies interaction between organisms, response to stress, regulation of cell death, and apoptosis (Fig. 5). We also analyzed the 109 up-only and the 176 down-only protein networks separately. Both networks are significantly associated with translation, RNA processing and splicing, and the proteasome, which further illustrates that these processes are perturbed by the viral infection (Fig. 6).

Translation is an essential step in viral replication and mRNA vaccine action. DS-affinity identified 19 eukaryotic translation initiation factors, with 15 thus far being confirmed autoAgs (Table 1). In particular, 8 of the 13 subunits of the human elF3 complex were found in the interactome of the NSP1 protein of SARS-CoV-2, and all 8 are known autoAgs (Table 1). elF3 is essential for the most forms of cap-dependent and cap-independent translation initiation and stimulates nearly all steps of translation initiation, as well as other phases of translation such as recycling. elF3 functions in a number of prominent human pathogens, e.g., HIV and HCV; and the present finding indicates that elF3 also functions in SARS-CoV-2 infection.

Among the 657 COVID-affected DS-affinity proteins, 369 (56\%) are thus far confirmed autoAgs, accounting for $92 \%$ of the 400 confirmed autoAgs of the master autoantigen-ome. This vast number of perturbed autoAgs demonstrates that COVID-19 could lead to a wide variety of autoimmune diseases. For example, 42 autoAgs are associated with the myelin sheath and many are associated with other components of the nervous system, as we have described previously, which may help explain a myriad of neurological symptoms caused by COVID-19 [1]. As another example, 11 autoAgs are related to stress fibers (contractile actin filament bundles consisting of short actin filaments with alternating polarity) and 25 proteins are associated with myofibrils (contractile elements of skeletal and cardiac muscle), which may explain various muscular and cardiomuscular sequelae of COVID-19.

A few autoAgs also interact with multiple viral proteins of SARS-CoV-2, suggesting that they play important roles in COVID-19 and merit further investigation. For example, ESYT1 and MOV10 interact with 12 viral proteins, CALU interacts with 11, HSPA5 interacts with 9, COPG1 and ARHGAP1 interact with 8, PLD3 and 
MARCKS interact with 7, and IDE interacts with 6 viral proteins (Table 1). PLD3 (a phospholipase) influences the processing of amyloid-beta precursor protein and is associated with spinocerebellar ataxia and Alzheimer's disease. IDE (insulin-degrading enzyme) degrades intracellular insulin and is associated with diabetes.

\section{AutoAg coding gene characteristics and alternative splicing}

To further understand the autoantigen-ome, we mapped the coding genes for 751 proteins of the master autoantigen-ome, and they are distributed over all chromosomes (Fig. 7). Since these include both confirmed and putative autoAgs, one may argue that some of the putative autoAgs may not be true and the gene characteristics may not be meaningful. Therefore, we also mapped the genes for the 400 confirmed autoAgs, and they are similarly distributed over all chromosomes (Fig. 7). For both confirmed and putative autoAgs, coding gene prevalence is significantly higher on chromosomes 11, 12, 17, and 19, lower on chromosome 18, and almost absent on chromosome $Y$ (Fig. 7). Various cluster loci are noticeable, e.g., on chromosomes 1, 11, 12, 17, and 19.

Intriguingly, autoAg coding genes contain significantly larger numbers of exons than expected, with the majority containing at least 4 exons (Fig. 8). The number of transcript isoforms per coding gene is also significantly skewed towards higher numbers, and those with $\geq 6$ isoforms are particularly dominant. Furthermore, the lengths of coding sequence, transcript, and $3^{\prime}$ and 5'-UTR of autoAg coding genes are skewed towards shorter sizes relative to the distribution of all coding genes (Fig. 8). We also examined the coding genes of the 400 confirmed autoAgs, and they show similar dominance in higher number ofexons and -isoforms, shorter transcripts, and shorter 3'-UTR lengths (Fig. 8).

The predominance of multiple exons and transcript variants suggests a role for RNA processing and alternative splicing in the origination of autoAgs. For genes with multiple exons, alternative splicing can yield a range of unique protein isoforms by varying the exon composition. Curiously, numerous components of the splicing machinery are well-known nuclear autoAgs. In fact, this study identified 120 potential autoAgs associated with RNA processing and 70 potential autoAgs associated with RNA splicing (Table 1 and Figs. 2-3). The majority of these have been found to be affected by SARS-CoV-2 infection (Figs. 5-6).

During splicing, a group of snRNPs (small nuclear ribonucleoproteins) bind to the intron of a newly formed pre-mRNA and splice it to result in a mature mRNA. Ten snRNP autoAgs are identified by DS-affinity, 8 of which have been found to be affected by SARS-CoV-2 infection (Table 1). During splicing, snRNAs undergo conformational rearrangements that are catalyzed by the DEAH/DEAD box superfamily of RNA helicases. 11 such helicases are identified by DS-affinity, and 10 have been found to be affected by the viral infection (Table 1). Serine/arginine-rich splicing factors, such as SRSF1 (also known as alternative splicing factor 1), are sequence-specific splicing factors involved in pre-mRNA splicing. 9 SRSF proteins are identified by DSaffinity, with 7 found to be affected by the viral infection. Seven additional splicing factors are identified by DS-affinity (e.g., poly(U)-binding splicing factor PUF60), with all found to be affected by SARS-CoV-2 infection. Heterogeneous nuclear ribonucleoproteins (hnRNPs) play various roles in gene transcription and post-transcriptional modification of pre-mRNA, e.g., binding pre-mRNAs to render splice sites more 
or less accessible to the spliceosome and suppressing RNA splicing at a particular exon. 19 hnRNP proteins are identified by DS-affinity, with 17 found affected by SARS-CoV-2 infection.

The large number of autoAgs of the RNA splicing machinery and their involvement in SARS-CoV-2 infection provide support to the notion that viral infections exploit alternative splicing. It is logical to speculate that viruses hijack the splicing machinery to force the host to synthesize virus-beneficial protein isoforms and thereby reprogram the host cellular protein network so that the virus can survive and replicate. It is also plausible that protein isoforms from virus-induced alternative splicing are recognizable by our immune system as unusual and non-self and hence may trigger an (auto)immune response.

Various studies have reported alternative splicing among autoAgs. For example, an informatics analysis of 45 autoAgs showed that alternative splicing occurred in $100 \%$ of the transcripts, which was significantly higher than the $\sim 42 \%$ rate observed in a randomly selected set of 9,554 gene transcripts. Furthermore, $80 \%$ of the transcripts underwent non-canonical alternative splicing, which was significantly higher than the $<1 \%$ rate in randomly selected human gene transcripts [38]. As another example, Ro52/SSA is one of the autoAg targets strongly associated with the autoimmune responses in mothers whose children have manifestations of neonatal lupus. The gene for full-length Ro52 spans $10 \mathrm{~kb}$ of DNA and contains 7 exons, and an alternatively spliced transcript encoding a novel autoAg expressed in the fetal and adult heart has been identified [39]. In a patient with primary Sjörgren syndrome, an alternative mRNA variant of the nuclear autoAg La/SSB was found to result from a promoter switch and alternative splicing [40].

\section{Common autoAgs associated with cell stress and apoptosis}

We have consistently found that DS binds apoptotic cells regardless of cell type $[6,8]$. To figure out which molecules are involved in this affinity, we searched for DS-affinity proteins shared in all 6 human cell lines of this study and found 39 autoAg candidates (Fig. 9). These include 9 ER chaperone complex proteins, 5 14-3-3 proteins, 3 hnRNPs, and 3 tropomyosin proteins. All are known autoAgs except for ANP32A and YWHAB (14-3-3 alpha/beta). Given that ANP32A's paralog ANP32B and 5 other 14-3-3 isoforms are known autoAgs, it is likely they are also true autoAgs. Remarkably, several classical ANA (antinuclear antibody) autoAgs that define systemic autoimmune diseases are among the autoAgs found in the DS-affinity proteomes of all 6 human cell lines, including histone H1 and H4, SSB (lupus La), XRCC5/Ku80, XRCC6/Ku70, and PCNA. Because these autoAgs are commonly found in apoptotic cells, it is not surprising that autoimmune responses targeting these autoAgs tend to be systemic; in other words, they all are potential markers of systemic autoimmune diseases.

Based on GO Biological Process and Reactome Pathway analysis, 22 of the common autoAgs are associated with cellular responses to stress, 17 are associated with regulation of apoptotic processes, and 8 are markers of apoptosis (Fig. 9). Moreover, these common autoAgs are involved in chromosome organization (ANP32A, ANP32B, H1-2, H4, KPNB1, NPM1, PCNA, SET, XRCC5, XRCC6), cytoskeleton organization (ACTN1, CALR, TPM1, TPM3, TPM4, TUBA1C, VIM), and mitochondrial membrane organization (YWHAB, YWHAE, YWHAG, YWHAQ, YWHAZ). These findings reveal that apoptosis is accompanied by reorganization of the nucleus, mitochondria, and cytoskeleton. 
Furthermore, 37 of the 39 common autoAgs were altered in SARS-CoV-2 infection. Based on GO Biological Process analysis, 13 of these proteins are involved in viral processing, namely, KPNB1, C1QBP, HSP90AB1, NPM1, SYNCRIP, SET, SSB, XRCC5, XRCC6, VCP, VIM, YWHAB, and YWHAE. These findings further support our model of linking viral infection to autoimmunity, with viral infections leading to host cell stress, cell death, autoimmune reactions, and eventually autoimmune diseases (Fig. 1).

\section{UBA1, $X$-inactivation escape, and female predilection of autoimmunity}

Among the above common autoAgs, UBA1 (or UBE1, ubiquitin-like modifier-activating enzyme 1) plays an essential role in dead cell clearance. UBA1 catalyzes the first step in ubiquitination - the "kiss of death" that marks cellular proteins for degradation. It has long been speculated that dysregulation of apoptotic pathways and dysfunctional clearance of dead cells are among the main causes of autoimmunity, which is in line with our findings $[6,8]$. Apoptosis also directly contributes to the maintenance of lymphocyte homeostasis and the deletion of autoreactive cells. Therefore, dysfunction of UBA1 could result in deficient clearance of apoptotic cells and aberrant autoimmunity.

Recently, UBA1 somatic mutations have been linked to a severe adult-onset autoinflammatory disease termed VEXAS syndrome [41]. A somatic mutation affecting methionine-41 in UBA1 results in a loss of the canonical cytoplasmic isoform of UBA1 and in the expression of a novel catalytically impaired isoform. Additionally, mutant peripheral blood cells show decreased ubiquitination and activated innate immune pathways.

Strikingly, UBA1 protein expression is found up-regulated at different time points of SARS-CoV-2 infection, whereas two deubiquitinating enzymes, USP9X and USP5, are down-regulated [33] (Supplemental Table 1). Furthermore, among the 657 proteins of the COVID autoantigen-ome, 178 have been found to be affected by ubiquitination (Fig. 10). They are most significantly associated with RNA metabolism and cellular response to stress. In addition, ubiquitination affects proteins involved in signaling by Rho GTPase, RNA splicing, translation, protein folding, nonsense-mediated decay, DNA damage stress-induced senescence, and the cytoskeleton. These findings underline the extensive involvement of ubiquitination in viral infection.

UBA1 is coded by the UBA1 gene located on the $X$ chromosome with no homolog on the $Y$ chromosome, and more importantly, UBA1 can escape X-chromosome inactivation. UBA1 appears to be protected against chromosome-wide transcriptional silencing by a chromatin boundary flanked by histone $\mathrm{H} 3$ modifications and CpG hypomethylation [42]. In human female fibroblasts, UBA1 mRNA is detected from both the active and inactive $X$ chromosomes, and UBA 1 is expressed in a large panel of somatic cell hybrids retaining inactive $X$ chromosomes [43]. In human endothelial cells from dizygotic twins, UBA1 and a few other X-chromosome encoded proteins are expressed at higher levels in female cells [44]. UBA1 expression is estimated to be $\sim 60 \%$ from $X$-active alleles, $30 \%$ biallelic, and $10 \%$ from $X$-inactive alleles [45].

X-linked genes, particularly escape genes, contribute to sex differences. In women, about $15 \%$ of X-linked genes are bi-allelically expressed, and expression from the inactive $X$ allele varies from a few percent to 
near equal to that of the active allele [46]. X-inactivation and escape may enhance phenotypic differences between females and males and may also enhance variability within females due to mosaicism from cells with the X-maternal or X-paternal inactivated and to a variable degree of escape from X-inactivation [46]. Aging, which is associated with telomere shortening, can relax $\mathrm{X}$-inactivation and force global transcriptome alterations [47], which may lead to gene escape and altered expression of UBA1. Therefore, dysfunction of UBA1 due to X-inactivation escape may predispose women, particularly aging women, to increasing dysfunctional regulation of apoptosis and aberrant autoimmunity.

\section{Considerations for vaccine design based on Spike-protein via viral vectors or mRNAs}

To understand the various rare but reported side effects from the currently available viral vector- and mRNA-encoded S-protein COVID vaccines, we searched for autoAgs that may interact with the spike protein of SARS-CoV-2 and found 15 autoAg candidates (Table 2). Of these, CALU, ESYT1, MOV10, and MARCKS may also interact with many other SARS-CoV-2 proteins as discussed earlier. Curiously, at least 2 of these are associated with blood clotting problems, and 5 are implicated in neurological disorders (Table 2). For example, CALU (calumenin) is a calcium-binding protein and is expressed in high levels in the heart, placenta, and skeletal muscle. CALU is associated with pharmacodynamics and response to elevated platelet cytosolic $\mathrm{Ca}^{2+}$, platelet degranulation, and Coumarin/Warfarin resistance. Warfarin is an anticoagulant (blood thinner) drug used to treat blood clots such as deep vein thrombosis and pulmonary embolism and to prevent stroke in people with heart problems such as atrial fibrillation, valvular heart disease or in people with artificial heart valves.

Table 2. Diseases associated with potential SARS-CoV-2 spike protein-interacting autoAgs*

\begin{tabular}{|c|c|}
\hline CALU & Warfarin (anti-coagulants for blot clotting) resistance disease \\
\hline ESYT1 & $\begin{array}{l}\text { Stormorken syndrome (mild bleeding tendency due to platelet dysfunction, thrombocytopenia, anemia, } \\
\text { asplenia, etc.) }\end{array}$ \\
\hline MOV10 & Viral infection, autism spectrum disorder \\
\hline MARCKS & Spinocerebellar ataxia 14, barbiturate dependence \\
\hline HSPBP1 & Autosomal recessive spinocerebellar ataxia 16, Sjögren-Larsson syndrome \\
\hline PRS27A & Machado-Joseph disease (spinocerebellar ataxia type III), spherocytosis type 5 \\
\hline EZR & Autosomal recessive non-syndromic intellectual disability, neurofibromatosis type II \\
\hline HSPA5 & Mucormycosis (fungal infection), Wolfram syndrome 1 (diabetes mellitus) \\
\hline ARHGAP1 & Noma, Lowe oculocerebrorenal syndrome (affects eyes, central nervous system and kidneys) \\
\hline MSN & X-linked moesin-associated immunodeficiency, verrucous carcinoma \\
\hline CSPG4 & Acral lentiginous melanoma, melanoma \\
\hline SLC1A5 & Hartnup disorder, placental choriocarcinoma \\
\hline PRKCSH & Polycystic liver disease \\
\hline CAVIN1 & Lipodystrophy, congenital generalized lipodystrophy \\
\hline BASP1 & Distal X-linked spinal muscular atrophy, Wilms tumor 1 \\
\hline
\end{tabular}

Although largely speculative at present, these potential S-protein-interacting autoAgs may provide partial explanations for the rare hematological, neurological, and muscular side effects reported for the currently available COVID vaccines (Table 2). Although it is known that $S$ proteins are synthesized intracellularly following vaccination with mRNAs or viral vectors, many of the precise molecular steps remain unknown. In particular, how do these newly synthesized $S$ proteins fold and are they glycosylated differently 
depending on the cell type that rakes up the mRNA or the viral vector? How does the newly synthesized $S$ protein interact with other host cell components before being processed (or degraded) and presented to immune cells? For example, could the nascent $S$ proteins interact with CALU or ESYT1 to cause blood clotting problems, could S protein interaction with HSPA5 contributes to fungal infection outbreaks as seen in India? These and many other questions await further investigation. This is of interest because mRNA and vector-based vaccines make use of a variety of cell types in vivo to produce the immunogen, whereas recombinant protein-based vaccines introduce the ex vivo prepared immunogen directly to the immune system.

In addition, this study identified a large number of autoAg candidates that are crucial for vector-based or mRNA vaccine action, including translation, RNA processing and metabolism, vesicles and vesiclemediated transport, and protein processing and transport (Figs. 2-6). For example, the master autoantigen-ome contains 56 ribosomal proteins, 16 eukaryotic translation initiation factors, 16 aminoacyl-tRNA synthases/ligases, and 6 translation elongation factors, all of which are essential actors in translating mRNAs into proteins. There are also many autoAgs related to protein folding and posttranslational protein modification, although it is not clear whether the $S$ proteins are folded and posttranslationally modified before being processed and presented to immune cells in the currently used mRNA or vector vaccines for COVID-19. These potential autoAgs may confer clues to understanding the observed rare adverse events and should help guide the future development of even safer vaccines.

\section{Conclusion}

In this report, we compiled a master autoantigen-ome of 751 potential autoAgs, 657 of which are affected in SARS-CoV-2 infection, and 400 of which are confirmed autoAgs in a wide variety of autoimmune diseases and cancer. Our proposed model (Fig. 1) provides a plausible explanation for how a cascade of molecular changes associated with viral infection leads to cell stress, apoptosis, and subsequent autoimmune responses. The large number of autoAg candidates associated with SARS-CoV-2 infection provides a mechanistic rationale for the close monitoring of autoimmune diseases that may follow the COVID-19 pandemic. In addition, the coding gene characteristics of autoAgs described in this study provide further insights into the genetic origination of autoAgs. The significance of ubiquitination in apoptotic cell clearance and protein turnover and the X-linked escape expression of UBA1 might explain, in part, the predisposition of aging women to autoimmune diseases.

\section{Materials and Methods}

\section{DS-affinity autoAg identification}

Potential autoAgs were identified by DS-affinity from protein extracts from six human cell lines as previously described, including HFL1 fetal lung fibroblasts [1], A549 lung epithelial cells [2], HS-Sultan Blymphoblasts [4], Wil2-NS B-lymphoblasts [7], Jurkat T-lymphoblasts [5], and HEp-2 fibroblasts [11]. 


\section{Autoantigen literature text mining}

Each DS-affinity protein was verified as to whether it is a target of autoantibodies by an extensive literature search on PubMed. Search keywords included the MeSH keyword "autoantibodies", the protein name or its gene symbol, or alternative names and symbols. Only proteins for which specific autoantibodies are reported in PubMed-listed journal articles were considered "confirmed" or "known" autoAgs in this study.

\section{COVID data comparison}

DS-affinity proteins were compared with currently available COVID-19 multi-omic data compiled in the Coronascape database (as of 05/27/2021) [16-37]. These data have been obtained with proteomics, phosphoproteomics, interactome and ubiquitome studies, and RNA-seq techniques. Up- and/or downregulated proteins or genes were identified by comparing cells infected vs. uninfected by SARS-CoV-2 or COVID-19 patients vs. healthy controls. Similarity searches were conducted to identify DS-affinity proteins that are similar to those found up- and/or down-regulated in the viral infection at any omic level.

\section{Protein network analysis}

Protein-protein interactions were analyzed with STRING [14]. Interactions include both direct physical interaction and indirect functional associations, which are derived from genomic context predictions, high-throughput lab experiments, co-expression, automated text mining, and previous knowledge in databases. Each interaction is annotated with a confidence score between 0 (lowest) and 1 (highest), indicating the likelihood of an interaction to be true. Enrichment of pathways and processes were analyzed with Metascape [16], which utilize various ontological sources such as KEGG Pathway, GO Biological Process, Reactome Gene Sets, and Canonical Pathways. All genes in the genome were used as the enrichment background. Terms with a $p$ value $<0.01$, a minimum count of 3 , and an enrichment factor (ratio between the observed counts and the counts expected by chance) $>1.5$ were grouped into clusters based on their membership similarities. The most statistically significant term within a cluster was chosen to represent the cluster.

\section{Gene characteristic analysis}

Gene characteristics were analyzed with ShinyGO [15]. ShinyGO is based on a large annotation database derived from Ensembl and STRING-db. The characteristics of the genes for the groups of autoAgs in this study were compared with the rest in the genome. Chi-squared and Student's t-tests were run to see if the autoAg genes had special characteristics when compared with all other genes in the human genome. 


\section{Funding Statement}

This work was partially supported by Curandis, the US NIH, and a Cycle for Survival Innovation Grant (to MHR). MHR acknowledges NIH/NCI R21 CA251992 and MSKCC Cancer Center Support Grant P30 CA008748. The funding bodies were not involved in the design of the study and the collection, analysis, and interpretation of data.

\section{Competing interest statement}

JYW is the founder and Chief Scientific Officer of Curandis. MHR is a member of the Scientific Advisory Boards of Trans-Hit, Proscia, and Universal DX, but these companies have no relation to the study.

\section{Authors' contributions}

JYW conducted the study and wrote the manuscript. MWR and VBR assisted with the study and manuscript preparation. MHR consulted on the study and edited the manuscript. All authors have approved the manuscript. 


\section{References}

[1] J. Y. Wang, W. Zhang, M. W. Roehrl, V. B. Roehrl, M. H. Roehrl. An Autoantigen Atlas from Human Lung HFL1 Cells Offers Clues to Neurological and Diverse Autoimmune Manifestations of COVID19. bioRxiv, 2021;10.1101/2021.01.24.427965.

[2] J. Y. Wang, W. Zhang, M. W. Roehrl, V. B. Roehrl, M. H. Roehrl. An autoantigen profile of human A549 lung cells reveals viral and host etiologic molecular attributes of autoimmunity in COVID-19. Journal of autoimmunity, 2021;120:102644.

[3] J. Y. Wang, W. Zhang, M. W. Roehrl, V. B. Roehrl, M. H. Roehrl. An Autoantigen Profile of Human A549 Lung Cells Reveals Viral and Host Etiologic Molecular Attributes of Autoimmunity in COVID19. bioRxiv, 2021;10.1101/2021.02.21.432171.

[4] J. Y. Wang, W. Zhang, V. B. Roehrl, M. W. Roehrl, M. H. Roehrl. An Autoantigen-ome from HSSultan B-Lymphoblasts Offers a Molecular Map for Investigating Autoimmune Sequelae of COVID19. bioRxiv, 2021;10.1101/2021.04.05.438500.

[5] J. Y. Wang, W. Zhang, M. W. Roehrl, V. B. Roehrl, M. H. Roehrl. An Autoantigen Profile from Jurkat T-Lymphoblasts Provides a Molecular Guide for Investigating Autoimmune Sequelae of COVID-19. bioRxiv, 2021;10.1101/2021.07.05.451199.

[6] J. Y. Wang, J. Lee, M. Yan, J. H. Rho, M. H. Roehrl. Dermatan sulfate interacts with dead cells and regulates $\mathrm{CD} 5(+)$ B-cell fate: implications for a key role in autoimmunity. Am J Pathol, 2011;178:2168-76.

[7] J. H. Rho, W. Zhang, M. Murali, M. H. Roehrl, J. Y. Wang. Human proteins with affinity for dermatan sulfate have the propensity to become autoantigens. Am J Pathol, 2011;178:2177-90.

[8] J. Lee, J.-h. Rho, M. H. Roehrl, J. Y. Wang. Dermatan Sulfate Is a Potential Regulator of IgH via Interactions With Pre-BCR, GTF2I, and BiP ER Complex in Pre-B Lymphoblasts. Frontiers in immunology, 2021;12.

[9] J. Lee, J.-h. Rho, M. H. Roehrl, J. Y. Wang. Dermatan Sulfate Is a Potential Master Regulator of IgH via Interactions with Pre-BCR, GTF2I, and BiP ER Complex in Pre-B Lymphoblasts. bioRxiv, 2021;10.1101/2021.01.18.427153.

[10] J. Y. Wang, M. H. Roehrl. Glycosaminoglycans are a potential cause of rheumatoid arthritis. Proceedings of the National Academy of Sciences of the United States of America, 2002;99:143627.

[11] J. Y. Wang, W. Zhang, J. H. Rho, M. W. Roehrl, M. H. Roehrl. A proteomic repertoire of autoantigens identified from the classic autoantibody clinical test substrate HEp-2 cells. Clinical proteomics, 2020;17:35.

[12] W. Zhang, J. H. Rho, M. H. Roehrl, J. Y. Wang. A comprehensive autoantigen-ome of autoimmune liver diseases identified from dermatan sulfate affinity enrichment of liver tissue proteins. BMC immunology, 2019;20:21.

[13] W. Zhang, J. H. Rho, M. W. Roehrl, M. H. Roehrl, J. Y. Wang. A repertoire of 124 potential autoantigens for autoimmune kidney diseases identified by dermatan sulfate affinity enrichment of kidney tissue proteins. PloS one, 2019;14:e0219018.

[14] D. Szklarczyk, A. L. Gable, D. Lyon, A. Junge, S. Wyder, J. Huerta-Cepas et al. STRING v11: proteinprotein association networks with increased coverage, supporting functional discovery in genome-wide experimental datasets. Nucleic acids research, 2019;47:D607-D13.

[15] S. X. Ge, D. Jung, R. Yao. ShinyGO: a graphical gene-set enrichment tool for animals and plants. Bioinformatics (Oxford, England), 2020;36:2628-9. 
[16] Y. Zhou, B. Zhou, L. Pache, M. Chang, A. H. Khodabakhshi, O. Tanaseichuk et al. Metascape provides a biologist-oriented resource for the analysis of systems-level datasets. Nature communications, 2019;10:1523.

[17] J. Y. Zhang, X. M. Wang, X. Xing, Z. Xu, C. Zhang, J. W. Song et al. Single-cell landscape of immunological responses in patients with COVID-19. Nature immunology, 2020;21:1107-18.

[18] J. P. Davies, K. M. Almasy, E. F. McDonald, L. Plate. Comparative Multiplexed Interactomics of SARS-CoV-2 and Homologous Coronavirus Nonstructural Proteins Identifies Unique and Shared Host-Cell Dependencies. ACS infectious diseases, 2020;6:3174-89.

[19] K. Klann, D. Bojkova, G. Tascher, S. Ciesek, C. Münch, J. Cinatl. Growth Factor Receptor Signaling Inhibition Prevents SARS-CoV-2 Replication. Molecular cell, 2020;80:164-74.e4.

[20] J. Sun, F. Ye, A. Wu, R. Yang, M. Pan, J. Sheng et al. Comparative Transcriptome Analysis Reveals the Intensive Early Stage Responses of Host Cells to SARS-CoV-2 Infection. Frontiers in microbiology, 2020;11:593857.

[21] D. Bojkova, K. Klann, B. Koch, M. Widera, D. Krause, S. Ciesek et al. Proteomics of SARS-CoV-2infected host cells reveals therapy targets. Nature, 2020;583:469-72.

[22] A. J. Wilk, A. Rustagi, N. Q. Zhao, J. Roque, G. J. Martínez-Colón, J. L. McKechnie et al. A single-cell atlas of the peripheral immune response in patients with severe COVID-19. Nature medicine, 2020;26:1070-6.

[23] N. A. P. Lieberman, V. Peddu, H. Xie, L. Shrestha, M. L. Huang, M. C. Mears et al. In vivo antiviral host transcriptional response to SARS-CoV-2 by viral load, sex, and age. PLoS biology, 2020;18:e3000849.

[24] L. Riva, S. Yuan, X. Yin, L. Martin-Sancho, N. Matsunaga, L. Pache et al. Discovery of SARS-CoV-2 antiviral drugs through large-scale compound repurposing. Nature, 2020;586:113-9.

[25] M. Bouhaddou, D. Memon, B. Meyer, K. M. White, V. V. Rezelj, M. Correa Marrero et al. The Global Phosphorylation Landscape of SARS-CoV-2 Infection. Cell, 2020;182:685-712.e19.

[26] D. Blanco-Melo, B. E. Nilsson-Payant, W. C. Liu, S. Uhl, D. Hoagland, R. Møller et al. Imbalanced Host Response to SARS-CoV-2 Drives Development of COVID-19. Cell, 2020;181:1036-45.e9.

[27] B. Shen, X. Yi, Y. Sun, X. Bi, J. Du, C. Zhang et al. Proteomic and Metabolomic Characterization of COVID-19 Patient Sera. Cell, 2020;182:59-72.e15.

[28] M. M. Lamers, J. Beumer, J. van der Vaart, K. Knoops, J. Puschhof, T. I. Breugem et al. SARS-CoV2 productively infects human gut enterocytes. Science (New York, NY), 2020;369:50-4.

[29] D. E. Gordon, G. M. Jang, M. Bouhaddou, J. Xu, K. Obernier, K. M. White et al. A SARS-CoV-2 protein interaction map reveals targets for drug repurposing. Nature, 2020;583:459-68.

[30] Y. Xiong, Y. Liu, L. Cao, D. Wang, M. Guo, A. Jiang et al. Transcriptomic characteristics of bronchoalveolar lavage fluid and peripheral blood mononuclear cells in COVID-19 patients. Emerging microbes \& infections, 2020;9:761-70.

[31] A. Vanderheiden, P. Ralfs, T. Chirkova, A. A. Upadhyay, M. G. Zimmerman, S. Bedoya et al. Type I and Type III Interferons Restrict SARS-CoV-2 Infection of Human Airway Epithelial Cultures. Journal of virology, 2020;94.

[32] S. Appelberg, S. Gupta, S. Svensson Akusjärvi, A. T. Ambikan, F. Mikaeloff, E. Saccon et al. Dysregulation in Akt/mTOR/HIF-1 signaling identified by proteo-transcriptomics of SARS-CoV-2 infected cells. Emerging microbes \& infections, 2020;9:1748-60.

[33] A. Stukalov, V. Girault, V. Grass, V. Bergant, O. Karayel, C. Urban et al. Multi-level proteomics reveals host-perturbation strategies of SARS-CoV-2 and SARS-CoV. bioRxiv, 2020;10.1101/2020.06.17.156455:2020.06.17.156455.

[34] W. Emanuel, M. Kirstin, F. Vedran, D. Asija, G. L. Theresa, A. Roberto et al. Bulk and single-cell gene expression profiling of SARS-CoV-2 infected human cell lines identifies molecular targets for therapeutic intervention. bioRxiv, 2020;10.1101/2020.05.05.079194:2020.05.05.079194. 
[35] Y. Li, Y. Wang, H. Liu, W. Sun, B. Ding, Y. Zhao et al. Urine Proteome of COVID-19 Patients. medRxiv : the preprint server for health sciences, 2020;10.1101/2020.05.02.20088666:2020.05.02.20088666.

[36] M. Liao, Y. Liu, J. Yuan, Y. Wen, G. Xu, J. Zhao et al. Single-cell landscape of bronchoalveolar immune cells in patients with COVID-19. Nature medicine, 2020;26:842-4.

[37] E. M. N. Laurent, Y. Sofianatos, A. Komarova, J.-P. Gimeno, P. S. Tehrani, D.-K. Kim et al. Global BiolD-based SARS-CoV-2 proteins proximal interactome unveils novel ties between viral polypeptides and host factors involved in multiple COVID19-associated mechanisms. bioRxiv, 2020;10.1101/2020.08.28.272955:2020.08.28.272955.

[38] B. Ng, F. Yang, D. P. Huston, Y. Yan, Y. Yang, Z. Xiong et al. Increased noncanonical splicing of autoantigen transcripts provides the structural basis for expression of untolerized epitopes. The Journal of allergy and clinical immunology, 2004;114:1463-70.

[39] E. K. Chan, F. Di Donato, J. C. Hamel, C. E. Tseng, J. P. Buyon. 52-kD SS-A/Ro: genomic structure and identification of an alternatively spliced transcript encoding a novel leucine zipper-minus autoantigen expressed in fetal and adult heart. The Journal of experimental medicine, 1995;182:983-92.

[40] M. Bachmann, M. Hilker, D. Grölz, G. Tellmann, U. Hake, L. Kater et al. Different La/SS-B mRNA isoforms are expressed in salivary gland tissue of patients with primary Sjögren's syndrome. Journal of autoimmunity, 1996;9:757-66.

[41] D. B. Beck, M. A. Ferrada, K. A. Sikora, A. K. Ombrello, J. C. Collins, W. Pei et al. Somatic Mutations in UBA1 and Severe Adult-Onset Autoinflammatory Disease. The New England journal of medicine, 2020;383:2628-38.

[42] Y. Goto, H. Kimura. Inactive $X$ chromosome-specific histone H3 modifications and CpG hypomethylation flank a chromatin boundary between an X-inactivated and an escape gene. Nucleic acids research, 2009;37:7416-28.

[43] L. Carrel, C. M. Clemson, J. M. Dunn, A. P. Miller, P. A. Hunt, J. B. Lawrence et al. X inactivation analysis and DNA methylation studies of the ubiquitin activating enzyme E1 and PCTAIRE-1 genes in human and mouse. Human molecular genetics, 1996;5:391-401.

[44] E. Witt, M. Lorenz, U. Völker, K. Stangl, E. Hammer, V. Stangl. Sex-specific differences in the intracellular proteome of human endothelial cells from dizygotic twins. Journal of proteomics, 2019;201:48-56.

[45] T. Tukiainen, A. C. Villani, A. Yen, M. A. Rivas, J. L. Marshall, R. Satija et al. Landscape of X chromosome inactivation across human tissues. Nature, 2017;550:244-8.

[46] J. B. Berletch, F. Yang, J. Xu, L. Carrel, C. M. Disteche. Genes that escape from X inactivation. Human genetics, 2011;130:237-45.

[47] S. Schoeftner, R. Blanco, I. Lopez de Silanes, P. Muñoz, G. Gómez-López, J. M. Flores et al. Telomere shortening relaxes $\mathrm{X}$ chromosome inactivation and forces global transcriptome alterations. Proceedings of the National Academy of Sciences of the United States of America, 2009;106:19393-8. 
bioRxiv preprint doi: https://doi.org/10.1101/2021.07.30.454526. this version posted August 4, 2021. The copyright holder for this preprint (which was not certified by peer review) is the author/funder, who has granted bioRxiv a license to display the preprint in perpetuity. It is made available under aCC-BY 4.0 International license.

Table 1. Autoantigens identified by DS-affinity and their alterations in SARS-CoV-2 infection

\begin{tabular}{|c|c|c|c|c|c|c|c|c|c|c|c|c|c|c|}
\hline \multirow[t]{2}{*}{$\mathbf{P}$} & \multirow{2}{*}{ Gene } & \multirow[t]{2}{*}{ Protein } & \multicolumn{6}{|c|}{ Cell line } & \multicolumn{3}{|c|}{$\begin{array}{l}\text { SARS-Cov-2 } \\
\text { infection }\end{array}$} & \multicolumn{2}{|c|}{$\begin{array}{c}\text { DS } \\
\text { affinity }\end{array}$} & \multirow[t]{2}{*}{ Ref. } \\
\hline & & & HFL1 & $\begin{array}{c}\text { HS- } \\
\text { Sultan }\end{array}$ & Wil2 & A549 & Jurkat & HEp-2 & $\mathbf{u}$ & d & interact. & hi & low & \\
\hline 5 & A2M & Alpha-2-macroglobulin & + & & & + & & & & $\mathrm{d}$ & & & + & [1] \\
\hline 6 & AARS & Alanine-tRNA ligase, AARS1 & + & + & & & & & u & d & & & + & [2] \\
\hline 15 & ACLY & ATP-citrate synthase & & + & & + & & & u & $\mathrm{d}$ & & & + & [3] \\
\hline 4 & ACTA1 & Actin, alpha skeletal muscle & & & + & & & & $\mathrm{u}$ & $d$ & & + & & [4] \\
\hline 10 & ACTA2 & Actin, aortic smooth muscle & + & + & & + & & + & $\mathrm{u}$ & $d$ & & + & + & [5] \\
\hline 8 & ACTB & Actin, cytoplasmic 1 & + & + & & + & & + & $\mathrm{u}$ & $d$ & & + & + & {$[6]$} \\
\hline 7 & ACTBL2 & Beta-actin-like protein & + & + & & + & & + & $\mathrm{u}$ & d & & + & + & [6] \\
\hline 2 & ACTBL3 & $\begin{array}{l}\text { Putative beta-actin-like protein } 3 \text {, kappa } \\
\text { actin, POTEKP }\end{array}$ & & & + & & + & & $\mathrm{u}$ & & & & + & \\
\hline 6 & ACTC1 & Actin, alpha cardiac muscle & & & & & + & & $\mathrm{u}$ & $d$ & & + & + & [7] \\
\hline 4 & ACTG1 & Actin, cytoplasmic 2 & & & + & & + & & $\mathrm{u}$ & $d$ & & + & + & [8] \\
\hline 28 & ACTN1 & Alpha-actinin-1 & + & + & + & + & + & + & $\mathrm{u}$ & $d$ & & & + & [9] \\
\hline 22 & ACTN4 & Alpha-actinin-4 & + & + & + & + & & + & $\mathrm{u}$ & $d$ & & & + & [5] \\
\hline 2 & ACTR2 & Actin-related protein 2 & & + & & & & & $\mathrm{u}$ & d & & & + & [10] \\
\hline 2 & ACTR3 & Actin-related protein 3 & & + & & & & & $\mathrm{u}$ & & & & + & [11] \\
\hline 2 & ADSS2 & $\begin{array}{l}\text { Adenylosuccinate synthetase isozyme } 2 \text {, } \\
\text { ADSS }\end{array}$ & & & & + & & & u & & & & + & \\
\hline 3 & AFP & Alpha-fetoprotein & + & + & & + & & & & $d$ & & & + & [12] \\
\hline 2 & AGRN & Agrin & & & & + & & & $\mathrm{u}$ & & $\begin{array}{c}\text { Nsp6 } \\
\text { Nsp13 } \\
\text { Orf8 } \\
\text { Orf10 }\end{array}$ & + & & [13] \\
\hline 15 & $\mathrm{AHCY}$ & Adenosylhomocysteinase, SAHH & & + & & + & & & & $d$ & & & + & {$[14]$} \\
\hline 5 & AHNAK & $\begin{array}{l}\text { Neuroblast differentiation-associated } \\
\text { protein }\end{array}$ & + & & & & & & u & $d$ & & & + & {$[15]$} \\
\hline 4 & AHSA1 & $\begin{array}{l}\text { Activator of } 90 \mathrm{kDa} \text { heat shock protein } \\
\text { ATPase homolog } 1\end{array}$ & & + & & & & & & $d$ & & & + & \\
\hline 2 & AHSG & Alpha-2-HS-glycoprotein, FETUA & & & & & & + & & $d$ & & & + & [16] \\
\hline 5 & AKR1B1 & Aldo-keto reductase family 1 member B1 & & & & + & & & $\mathrm{u}$ & $d$ & Orf3 & & + & [17] \\
\hline 10 & ALB & Albumin & + & & & + & & & $\mathrm{u}$ & $d$ & & + & + & [18] \\
\hline 5 & ALDH18A1 & Delta-1-pyrroline-5-carboxylate synthetase & & + & & & + & & & $\mathrm{d}$ & & & + & \\
\hline 23 & ALDH1A1 & Retinal dehydrogenase 1 & & & & + & & & $\mathrm{u}$ & $\mathrm{d}$ & & & + & [19] \\
\hline 5 & ALDH2 & Aldehyde dehydrogenase, mitochondrial & & & & + & & & $\mathrm{u}$ & $d$ & $\begin{array}{c}\text { E } \\
\text { Nsp5 } \\
\text { Nsp12 } \\
\text { Nsp16 }\end{array}$ & & + & [20] \\
\hline 5 & ALDH3A1 & Aldehyde dehydrogenase 3, ALDH3 & & & & + & & & u & $d$ & & & + & \\
\hline 9 & ALDOA & Fructose-bisphosphate aldolase A & & + & & + & & & $\mathrm{u}$ & d & & & + & [21] \\
\hline 4 & ALDOC & Fructose-bisphosphate aldolase C & & + & & & & & $\mathrm{u}$ & d & & & + & [22] \\
\hline 3 & ALPP & $\begin{array}{l}\text { Alkaline phosphatase, placental type } \\
\text { precursor }\end{array}$ & + & & & & & & & & & + & & [23] \\
\hline 10 & ANP32A & $\begin{array}{l}\text { Acidic leucine-rich nuclear phosphoprotein } \\
32 \text { member A }\end{array}$ & + & + & + & + & + & + & $\mathrm{u}$ & $d$ & & + & + & \\
\hline 13 & ANP32B & ANP 32 family member B & + & + & + & + & + & + & & $d$ & $\mathrm{~N}$ & + & + & [24] \\
\hline 3 & ANP32C & ANP 32 family member C, PP32R1 & + & + & & + & & & & & & + & + & \\
\hline 4 & ANP32E & ANP 32 family member $E$ & + & & + & + & & & $\mathrm{u}$ & $d$ & Orf9c & + & + & \\
\hline 4 & ANXA2 & Annexin A2 & + & & & + & & & $\mathrm{u}$ & d & & + & + & [25] \\
\hline 13 & ANXA2P2 & $\begin{array}{l}\text { Putative annexin A2-like protein, ANX2L2, } \\
\text { LPC2B }\end{array}$ & + & & & + & & & u & $d$ & & & + & [26] \\
\hline 10 & ANXA3 & Annexin A3 & & & & + & & & u & $d$ & & & + & [25] \\
\hline 5 & ANXA4 & Annexin IV & & & & + & & & $u$ & $\mathrm{~d}$ & & & + & [27] \\
\hline 15 & ANXA5 & Annexin A5 & + & + & & + & & & u & $d$ & Orf3 & & + & [28] \\
\hline 33 & ANXA6 & Annexin VI & + & + & + & + & + & & $u$ & $d$ & & & + & [29] \\
\hline 2 & AP1B1 & AP-1 complex subunit beta- 1 & + & & & & & & & & & & + & \\
\hline 8 & AP3B1 & AP-3 complex subunit beta- 1 & + & & & + & & & $\mathrm{u}$ & & $E$ & + & & \\
\hline 2 & AP3B2 & AP-3 complex subunit beta-2 & + & & & + & & & & & & + & & [30] \\
\hline 8 & AP3D1 & AP-3 complex subunit delta- 1 & + & & & + & & & $\mathrm{u}$ & $d$ & & + & & \\
\hline 4 & APEH & Acylamino-acid-releasing enzyme & & + & & & & & & & & & + & \\
\hline 4 & APEX1 & DNA-(apurinic or apyrimidinic site) lyase & & + & & + & & & $\mathrm{u}$ & $d$ & & & + & [31] \\
\hline 2 & API5 & Apoptosis inhibitor 5 & & + & + & & & & & $\mathrm{d}$ & & & + & \\
\hline 3 & APOA1 & Apolipoprotein A-I & + & & & & & & & $d$ & & & + & [32] \\
\hline
\end{tabular}


bioRxiv preprint doi: https://doi.org/10.1101/2021.07.30.454526; this version posted Auqust 4, 2021. The copyright holder for this preprint (which was not certified by peer review) is the author/funder, who has granted bioRxiv a license to display the preprint in perpetuity. It is made available under aCC-BY 4.0 International license.

\begin{tabular}{|c|c|c|c|c|c|c|c|c|c|c|c|c|c|c|}
\hline 2 & APOD & Apolipoprotein D & + & & & & & & $\mathrm{u}$ & $d$ & & & + & \\
\hline 8 & ARF1 & ADP-ribosylation factor & + & + & & & & & & & Nsp6 & & + & \\
\hline 2 & ARHGAP1 & Rho-GTPase-activating protein 1 & + & & & & & & $\mathrm{u}$ & & $\begin{array}{c}\text { Orf3a } \\
\text { Orf3b } \\
\text { Orf6 } \\
\text { Orf7a } \\
\text { Orf7b } \\
\text { Orf8 } \\
\text { Orf9c } \\
\text { S }\end{array}$ & & + & \\
\hline 6 & ARHGDIA & Rho GDP-dissociation inhibitor 1 & + & + & & & & & $\mathrm{u}$ & $d$ & & & + & \\
\hline 8 & ARHGDIB & Rho GDP-dissociation inhibitor 2 & & + & & & & & & $d$ & & & + & [33] \\
\hline 3 & ARPC2 & Actin-related protein $2 / 3$ complex subunit 2 & & + & & & & & & $d$ & & & + & \\
\hline 7 & ASMTL & $\begin{array}{l}\mathrm{N}-\text { Acetylserotonin O-methyltransferase- } \\
\text { like protein }\end{array}$ & & & + & & & & & & & & + & \\
\hline 2 & ASNS & $\begin{array}{l}\text { Glutamine-dependent asparagine } \\
\text { synthetase }\end{array}$ & & + & & & & & $\mathrm{u}$ & & & & + & \\
\hline 4 & ASPH & Aspartyl/asparaginyl beta-hydroxylase & & & & + & & & $\mathrm{u}$ & $d$ & Orf9c & + & & \\
\hline 14 & ATIC & $\begin{array}{l}\text { Bifunctional purine biosynthesis protein, } \\
\text { PURH }\end{array}$ & & + & + & + & & & & & & & + & [34] \\
\hline 2 & ATP2A2 & Sarcoplasmic/ER calcium ATPase 2 & & & & + & & & $u$ & & Nsp4 & + & & [35] \\
\hline 13 & ATP5F1B & $\begin{array}{l}\text { ATP synthase subunit beta, mitochondrial, } \\
\text { ATP5B }\end{array}$ & + & + & + & + & + & & $\mathrm{u}$ & $d$ & $\begin{array}{l}\text { Nsp6 } \\
\text { Orf9b }\end{array}$ & + & + & [36] \\
\hline 3 & ATXN10 & $\begin{array}{l}\text { Ataxin-10, Spinocerebellar ataxia type } 10 \\
\text { protein }\end{array}$ & & + & & & & & $\mathrm{u}$ & $d$ & & & + & \\
\hline 3 & BASP1 & $\begin{array}{l}\text { Brain acid soluble protein } 1 \text { (Neuronal } \\
\text { axonal membrane protein NAP22) }\end{array}$ & & & & & & + & $\mathrm{u}$ & $d$ & $\begin{array}{c}M \\
\text { Orf3a } \\
\text { Orf7b } \\
\text { S }\end{array}$ & & + & \\
\hline 3 & BCAT1 & $\begin{array}{l}\text { Branched chain amino acid } \\
\text { aminotransferase }\end{array}$ & + & & & & & & $\mathrm{u}$ & & & & + & \\
\hline 2 & $\mathrm{BCCIP}$ & BRCA2 and CDKN1A-interacting protein & + & & & & & & & & & & + & \\
\hline 2 & BGN & Biglycan & + & & & & & & & & & + & & [37] \\
\hline 3 & BRIX1 & $\begin{array}{l}\text { Ribosome biogenesis protein BRX1 } \\
\text { homolog }\end{array}$ & & & & + & & & & & & + & & \\
\hline 2 & BSG & Basigin, CD147 & + & & & & & & & $d$ & & + & & [38] \\
\hline 3 & BTF3 & Transcription factor BTF3, NACB & & & + & & & & $\mathrm{u}$ & $d$ & & & + & \\
\hline 2 & BZW1 & $\begin{array}{l}\text { Basic leucine zipper and W2 domain- } \\
\text { containing protein } 1\end{array}$ & & + & & & & & $\mathrm{u}$ & & & & + & \\
\hline 3 & BZW2 & $\begin{array}{l}\text { Basic leucine zipper and W2 domain- } \\
\text { containing protein } 2\end{array}$ & + & + & & & + & & & & M & & + & \\
\hline 7 & C1QBP & Complement C1q-binding protein & + & + & + & + & + & + & & $d$ & & + & + & [39] \\
\hline 7 & CALD1 & Caldesmon & + & & & & & & & $d$ & & & + & [40] \\
\hline 8 & CALM1 & Calmodulin-1 & + & + & & & + & + & $\mathrm{u}$ & $d$ & & & + & [41] \\
\hline 5 & CALM2 & Calmodulin-2 & & & + & & & & & d & & & + & \\
\hline 2 & CALM3 & Calmodulin-3 & & & + & & + & & $\mathrm{u}$ & & & & + & [42] \\
\hline 19 & CALR & Calreticulin & + & + & + & + & + & + & u & $d$ & & & + & {$[43]$} \\
\hline 2 & CALU & Calumenin & + & & & & & & $\mathrm{u}$ & $d$ & $\begin{array}{c}E \\
M \\
\text { Nsp6 } \\
\text { Nsp7 } \\
\text { Orf3a } \\
\text { Orf3b } \\
\text { Orf6 } \\
\text { Orf7a } \\
\text { Orf7b } \\
\text { Orf9c } \\
\text { S }\end{array}$ & & + & [44] \\
\hline 15 & CAND1 & $\begin{array}{l}\text { Cullin-associated NEDD8-dissociated } \\
\text { protein } 1\end{array}$ & & + & + & & + & & & & & & + & \\
\hline 7 & CANX & Calnexin & + & & & + & & + & $\mathrm{u}$ & $d$ & $\begin{array}{l}\text { Nsp4 } \\
\text { Orf8 }\end{array}$ & + & & [45] \\
\hline 9 & CAP1 & Adenylyl cyclase-associated protein 1 & + & + & & + & & & $\mathrm{u}$ & $d$ & Orf3 & & + & \\
\hline 7 & CAPN1 & Calpain- 1 catalytic subunit & + & + & & + & & & & & & & + & \\
\hline 5 & CAPN2 & Calpain-2 catalytic subunit & + & & & & & + & $u$ & $d$ & Nsp16 & & + & [41] \\
\hline 3 & CAPNS1 & Calpain small subunit 1 & + & & & & & & & & & & + & \\
\hline 3 & CAPRIN1 & Caprin-1 & & + & + & & + & & & $d$ & & & + & \\
\hline 3 & CAPZA1 & F-actin-capping protein subunit alpha-1 & + & + & & & + & & & d & & + & + & [46] \\
\hline
\end{tabular}


bioRxiv preprint doi: https://doi.org/10.1101/2021.07.30.454526; this version posted Auqust 4, 2021. The copyright holder for this preprint (which was not certified by peer review) is the author/funder, who has granted bioRxiv a license to display the preprint in perpetuity. It is made available under aCC-BY 4.0 International license.

\begin{tabular}{|c|c|c|c|c|c|c|c|c|c|c|c|c|c|c|}
\hline 3 & CAPZB & F-actin-capping protein subunit beta & + & + & & + & & & & $d$ & & & + & [47] \\
\hline 8 & CAVIN1 & Caveolae-associated protein $1, \mathrm{PTRF}$ & + & & & & & & $\mathrm{u}$ & d & $\begin{array}{l}\mathrm{N} \\
\mathrm{S}\end{array}$ & & + & [48] \\
\hline 3 & CBX1 & Chromobox protein homolog 1 & + & & + & & & & $\mathrm{u}$ & & & & + & [49] \\
\hline 3 & CBX3 & Chromobox protein homolog 3 & & & + & & & & $\mathrm{u}$ & $d$ & & & + & \\
\hline 3 & CCDC6 & Coiled-coil domain-containing protein 6 & + & & & & & & $\mathrm{u}$ & $d$ & & & + & {$[50]$} \\
\hline 12 & CCT2 & T-complex protein 1 subunit beta & + & + & + & & + & & & $d$ & $\begin{array}{c}\text { Nsp12 } \\
\text { Orf8 } \\
\text { Orf9b } \\
\text { Orf10 }\end{array}$ & & + & {$[51]$} \\
\hline 12 & CCT3 & T-complex protein 1 subunit gamma & & + & + & & + & & u & & $\begin{array}{l}\text { Orf8 } \\
\text { Orf10 }\end{array}$ & & + & [52] \\
\hline 6 & CCT4 & T-complex protein 1 subunit delta & & + & + & & + & & $\mathrm{u}$ & & & & + & {$[52]$} \\
\hline 10 & CCT5 & T-complex protein 1 subunit epsilon & & + & + & & + & & $\mathrm{u}$ & $\mathrm{d}$ & $\begin{array}{c}\text { Nsp1 } \\
\text { Nsp12 } \\
\text { Orf8 } \\
\text { Orf10 }\end{array}$ & & + & [51] \\
\hline 7 & CCT6A & T-complex protein 1 subunit zeta & & + & + & & + & & $\mathrm{u}$ & $\mathrm{d}$ & $\begin{array}{c}\text { Nsp1 } \\
\text { Nsp12 } \\
\text { Orf10 }\end{array}$ & & + & {$[51]$} \\
\hline 9 & CCT7 & T-complex protein 1 subunit eta & & + & + & & + & & & & Orf10 & & + & {$[51]$} \\
\hline 20 & CCT8 & T-complex protein 1 subunit theta & + & + & + & + & + & & $\mathrm{u}$ & $\mathrm{d}$ & $\begin{array}{c}\text { Nsp1 } \\
\text { Nsp12 } \\
\text { Nsp14 } \\
\text { Nsp15 }\end{array}$ & & + & [52] \\
\hline 4 & CD248 & Endosialin & + & & & & & & & $d$ & & & + & \\
\hline 7 & CDC37 & Hsp90 co-chaperone Cdc37 & + & + & + & & + & & $u$ & d & Nsp16 & & + & \\
\hline 3 & CDK11A & Cyclin-dependent kinase 11A, CDC2L2 & & & & + & & & $\mathrm{u}$ & & & + & & \\
\hline 3 & CEBPZ & CCAAT/enhancer-binding protein zeta & & & & + & & & $\mathrm{u}$ & & & + & & \\
\hline 2 & CFL1 & Cofilin-1, CFL & & & + & & & & $\mathrm{u}$ & d & & & + & [53] \\
\hline 4 & CKAP4 & Cytoskeleton-associated protein 4, P63 & + & & & & & & $\mathrm{u}$ & $\mathrm{d}$ & $\begin{array}{l}\text { Nsp2 } \\
\text { Orf7b }\end{array}$ & + & & [54] \\
\hline 8 & CKB & Creatine kinase B-type & & & & & & & u & $\mathrm{d}$ & & & + & [55] \\
\hline 7 & CLIC1 & Chloride intracellular channel protein 1 & + & + & + & + & & & $u$ & d & Nsp16 & & + & {$[56]$} \\
\hline 2 & CLIC4 & Chloride intracellular channel protein 4 & + & & & & & & $\mathrm{u}$ & $d$ & & & + & \\
\hline 51 & CLTC & Clathrin heavy chain 1 & + & + & & + & & + & $u$ & d & & + & + & [57] \\
\hline 4 & CLTCL1 & Clathrin heavy chain 2 & + & + & & + & & + & & & & + & + & \\
\hline 4 & $\mathrm{CLUH}$ & $\begin{array}{l}\text { Clustered mitochondria protein homolog } \\
\text { (mRNA-binding) }\end{array}$ & & + & & & & & & $d$ & $\begin{array}{l}\text { Nsp7 } \\
\text { Nsp16 }\end{array}$ & & + & \\
\hline 2 & CMPK1 & UMP-CMP kinase & & + & & & & & & $d$ & & & + & \\
\hline 3 & CNDP2 & Cytosolic non-specific dipeptidase & & + & & & & & $\mathrm{u}$ & & $\begin{array}{l}\text { Orf3 } \\
\text { Orf10 }\end{array}$ & & + & \\
\hline 3 & CNPY2 & Protein canopy homolog & + & + & + & & & & & $d$ & Orf3a & & + & \\
\hline 13 & COL12A1 & Collagen type XII alpha-1 chain & + & & & & & & $\mathrm{u}$ & $d$ & & & + & \\
\hline 45 & COL1A1 & Collagen type I alpha- 1 chain & + & & & & & & $u$ & $d$ & & & + & [58] \\
\hline 37 & COL1A2 & Collagen type I alpha-2 chain & + & & & & & & & $d$ & & & + & [59] \\
\hline 2 & COL2A1 & Collagen type II alpha- 1 chain & + & & & & & & $\mathrm{u}$ & & & & + & {$[60]$} \\
\hline 12 & COL3A1 & Collagen type III alpha- 1 chain & + & & & & & & & & & & + & {$[61]$} \\
\hline 3 & COL5A1 & Collagen type $\mathrm{V}$ alpha 1 & + & & & & & & $\mathrm{u}$ & & & & + & [62] \\
\hline 6 & COL6A1 & Collagen type VI alpha-1 chain & + & & & & & & & $d$ & Orf8 & & + & [63] \\
\hline 4 & COL6A2 & Collagen type VI alpha-2 chain & + & & & & & & & d & & & + & \\
\hline 29 & COL6A3 & Collagen type VI alpha- 3 chain & + & & & & & & & $d$ & & & + & \\
\hline 2 & COPA & Coatomer subunit alpha & + & & & + & & & $\mathrm{u}$ & d & & + & & [64] \\
\hline 2 & COPB1 & Coatomer subunit beta & + & & & & & & & d & Nsp7 & + & & [65] \\
\hline 5 & COPB2 & Coatomer subunit beta' & + & & & & & + & u & & & + & & {$[66]$} \\
\hline 2 & COPD & Coatomer subunit delta, ARCN1 & + & & & & & & & d & $\begin{array}{l}\text { Orf3b } \\
\text { Orf6 }\end{array}$ & & + & \\
\hline 2 & COPG1 & Coatomer subunit gamma-1 & & & & & & + & & & $\begin{array}{c}\mathrm{E} \\
\mathrm{M} \\
\text { Nsp4 } \\
\text { Nsp6 } \\
\text { Orf3b } \\
\text { Orf6 } \\
\text { Orf7a } \\
\text { Orf7b }\end{array}$ & & + & [39] \\
\hline 2 & COPZ1 & Coatomer subunit zeta-1 & + & + & & & & & & $d$ & & & + & \\
\hline 12 & CORO1A & Coronin-1A & & + & & & & & $\mathrm{u}$ & & & & + & [67] \\
\hline
\end{tabular}


bioRxiv preprint doi: https://doi.org/10.1101/2021.07.30.454526: this version posted Auqust 4, 2021. The copyright holder for this preprint (which was not certified by peer review) is the author/funder, who has granted bioRxiv a license to display the preprint in perpetuity. It is made available under aCC-BY 4.0 International license.

\begin{tabular}{|c|c|c|c|c|c|c|c|c|c|c|c|c|c|c|}
\hline 3 & CORO1C & Coronin-1C & + & & & & & & & & & & + & \\
\hline 3 & CPNE1 & Copine-1 & & + & & & & & & & & & + & \\
\hline 4 & CPNE3 & Copine-3 & & + & & + & & & $\mathrm{u}$ & $d$ & & & + & \\
\hline 4 & CRK & Proto-oncogene c-Crk & + & & & & & & $\mathrm{u}$ & $d$ & $\begin{array}{l}\text { Nsp12 } \\
\text { Nsp14 } \\
\text { Nsp15 }\end{array}$ & & + & \\
\hline 5 & CRTAP & Cartilage-associated protein, $\mathrm{P} 3 \mathrm{H} 5$ & + & & & & & & & d & & + & & \\
\hline 3 & CS & Citrate synthase, mitochondrial & & + & & & & & $\mathrm{u}$ & $\mathrm{d}$ & $\mathrm{E}$ & & + & [3] \\
\hline 4 & CSK & Tyrosine-protein kinase CSK & & + & & & & & & $d$ & & & + & [68] \\
\hline 3 & CSNK2A1 & Casein kinase 2, alpha 1 & & & + & & & & & & & & + & \\
\hline 4 & CSPG4 & Chondroitin sulfate proteoglycan 4 & + & & & & & & & $\mathrm{d}$ & $\begin{array}{l}\text { Orf7b } \\
\mathrm{S}\end{array}$ & + & & [69] \\
\hline 4 & CTCFL & $\begin{array}{l}\text { High mobility group box } 1 \text { pseudogene } 1 \text {, } \\
\text { HMGB1P1, HMGB1L1 }\end{array}$ & + & & & & & & & & & & + & {$[136]$} \\
\hline 2 & CTR9 & $\begin{array}{l}\text { RNA polymerase-associated protein CTR9 } \\
\text { homolog }\end{array}$ & & & & + & & & $\mathrm{u}$ & $d$ & Orf9c & + & & \\
\hline 3 & CTSB & Cathepsin B, APP secretase & + & & & & & & $\mathrm{u}$ & $d$ & $\begin{array}{c}M \\
\text { Nsp12 }\end{array}$ & & + & \\
\hline 2 & CTSD & Cathepsin D & + & & & & & & $\mathrm{u}$ & $d$ & & & + & {$[70]$} \\
\hline 2 & CUTA & Protein $\underline{\text { CutA }}$ & + & & & & & & $\mathrm{u}$ & d & & & + & \\
\hline 6 & DAP3 & $\begin{array}{l}28 S \text { ribosomal protein S29, mitochondrial, } \\
\text { MRPS29 }\end{array}$ & & & & + & & & & & & + & & \\
\hline 6 & DARS & Aspartate-tRNA ligase, DARS1 & & + & & & & & & & & & + & [71] \\
\hline 2 & DBN1 & Drebrin 1 & + & & & & & & $\mathrm{u}$ & $d$ & & & + & [72] \\
\hline 4 & DCAF1 & $\begin{array}{l}\text { DDB1- and CUL4-associated factor } 1, \\
\text { VPRBP }\end{array}$ & & & & + & & & $\mathrm{u}$ & $d$ & & + & & \\
\hline 3 & DCK & Deoxycytidine kinase & & + & & & & & $\mathrm{u}$ & & & & + & \\
\hline 3 & DCN & Decorin & + & & & & & & & $d$ & & + & & [73] \\
\hline 2 & DCTN1 & $\begin{array}{l}\text { Dynactin subunit } 1,150 \mathrm{KDa} \text { Dynein- } \\
\text { associated protein }\end{array}$ & + & & & & & & & $d$ & & + & & {$[74]$} \\
\hline 5 & DCTN2 & Dynactin subunit 2 & + & + & & & & & & & Orf6 & & + & \\
\hline 3 & DCTPP1 & dCTP pyrophosphatase 1 & & + & & & & & & d & Orf9b & & + & \\
\hline 28 & DDB1 & DNA damage-binding protein 1 & + & + & & + & + & + & u & d & & + & + & [57] \\
\hline 3 & DDX17 & ATP-dependent RNA helicase DDX17 & & & & + & & & u & d & & + & & [53] \\
\hline 7 & DDX18 & ATP-dependent RNA helicase DDX18 & & & & + & & & $\mathrm{u}$ & & & + & & \\
\hline 5 & DDX21 & Nucleolar RNA helicase 2 & & & + & + & & & u & d & $\mathrm{N}$ & + & & {$[75]$} \\
\hline 4 & DDX27 & ATP-dependent RNA helicase DDX27 & & & & + & & & $\mathrm{u}$ & & & + & & \\
\hline 3 & DDX30 & ATP-dependent RNA helicase DHX30 & & & & + & & & & $d$ & & + & & \\
\hline 7 & DDX39A & ATP-dependent RNA helicase DDX39A & + & + & + & & + & & u & d & & & + & [39] \\
\hline 5 & DDX39B & Spliceosome RNA helicase BAT1 & + & + & + & & + & & & d & & & + & \\
\hline 4 & DDX5 & ATP-dependent RNA helicase, p68 & & + & & + & & & $\mathrm{u}$ & $\mathrm{d}$ & & + & + & [76] \\
\hline 16 & DDX9 & ATP-dependent RNA helicase A, DHX9 & + & + & + & + & & + & & & & + & + & {$[77]$} \\
\hline 2 & DEK & Protein DEK & & & + & & & & $\mathrm{u}$ & $\mathrm{d}$ & & & + & [53] \\
\hline 12 & DHX15 & $\begin{array}{l}\text { Pre-mRNA-splicing factor ATP-dependent } \\
\text { RNA helicase }\end{array}$ & + & + & & + & + & & & $\mathrm{d}$ & & + & + & \\
\hline 4 & DHX36 & ATP-dependent RNA helicase DHX36 & & & & + & & & $\mathrm{u}$ & & & + & & \\
\hline 5 & DIABLO & $\begin{array}{l}\text { Second mitochondria-derived activator of } \\
\text { caspase }\end{array}$ & + & + & + & & & & $\mathrm{u}$ & $\mathrm{d}$ & $\begin{array}{l}\text { Nsp6 } \\
\text { Nsp15 }\end{array}$ & & + & \\
\hline 4 & DKC1 & $\begin{array}{l}\text { H/ACA ribonucleoprotein complex subunit } \\
\text { B }\end{array}$ & + & & & + & & & $\mathrm{u}$ & $\mathrm{d}$ & & + & & \\
\hline 4 & DLD & $\begin{array}{l}\text { Dihydrolipoyl dehydrogenase, } \\
\text { mitochondrial }\end{array}$ & & + & & & & & & & & & + & [79] \\
\hline 2 & DLST & $\begin{array}{l}\text { Dihydrolipoyllysine-residue } \\
\text { succinyltransferase component of } 2 \text { - } \\
\text { oxoglutarate dehydrogenase complex }\end{array}$ & + & & & & & & & $d$ & & & + & [80] \\
\hline 2 & DNAJB11 & $\begin{array}{l}\text { DnaJ (Hsp40) homolog subfamily B } \\
\text { member } 11\end{array}$ & + & & & & & & $\mathrm{u}$ & & & & + & [81] \\
\hline 2 & DNAJC8 & DnaJ homolog subfamily $\mathrm{C}$ member 8 & & + & & & & & $\mathrm{u}$ & & & & + & \\
\hline 4 & DNPH1 & $\begin{array}{l}\text { 2'-deoxynucleoside 5'-phosphate N- } \\
\text { hydrolase } 1\end{array}$ & & + & & & & & $\mathrm{u}$ & & & & + & \\
\hline 6 & DPP3 & Dipeptidyl-peptidase 3 & + & + & & + & & & & $d$ & & & + & \\
\hline 3 & DPYSL2 & Dihydropyrimidinase-related protein & + & & & & & & $\mathrm{u}$ & d & & & + & {$[82]$} \\
\hline 3 & DRG1 & $\begin{array}{l}\text { Developmentally-regulated GTP-binding } \\
\text { protein }\end{array}$ & + & & & & & & & $\mathrm{d}$ & & & + & \\
\hline 5 & DUT & $\begin{array}{l}\text { Deoxyuridine } 5 \text { '-triphosphate } \\
\text { nucleotidohydrolase, mitochondrial }\end{array}$ & & + & & & & & $\mathrm{u}$ & $\mathrm{d}$ & & & + & \\
\hline 5 & DYNC1H1 & Dynein cytoplasmic 1 heavy chain 1 & + & & & & & & & & & + & & \\
\hline
\end{tabular}


bioRxiv preprint doi: https://doi.org/10.1101/2021.07.30.454526; this version posted August 4, 2021. The copyright holder for this preprint (which was not certified by peer review) is the author/funder, who has granted bioRxiv a license to display the preprint in perpetuity. It is made available under aCC-BY 4.0 International license.

\begin{tabular}{|c|c|c|c|c|c|c|c|c|c|c|c|c|c|c|}
\hline 3 & DYNC112 & Dynein cytoplasmic 1 intermediate chain 2 & + & & & + & & & & & & + & & \\
\hline 3 & EBP2 & $\begin{array}{l}\text { Probable rRNA-processing protein, } \\
\text { EBNA1BP2 }\end{array}$ & & & & + & & & & & & + & & \\
\hline 4 & $\mathrm{ECH} 1$ & $\begin{array}{l}\text { Delta(3,5)-delta(2,4)-dienoyl-CoA } \\
\text { isomerase, mitochondrial }\end{array}$ & & & & + & & & $\mathrm{u}$ & d & & & + & [83] \\
\hline 2 & EEF1A1 & Elongation factor 1 -alph 1 & + & + & & + & & & $\mathrm{u}$ & $d$ & & & + & [84] \\
\hline 4 & EEF1A2 & Elongation factor 1 -alpha 2 & + & + & & + & & & $\mathrm{u}$ & & Orf3 & & + & [85] \\
\hline 2 & EEF1B2 & Elongation factor 1 -beta 2 & + & + & + & & + & & & d & & & + & \\
\hline 5 & EEF1D & Elongation factor 1-delta & + & + & + & & & & & d & & & + & [86] \\
\hline 10 & EEF1G & Elongation factor 1-gamma & + & + & + & + & + & & $\mathrm{u}$ & d & & & + & \\
\hline 17 & EEF2 & Elongation factor 2 & + & + & + & + & & & $\mathrm{u}$ & d & & + & + & [87] \\
\hline 16 & EFTUD2 & 116 kDa U5 snRNP component, SNRP116 & + & + & + & + & & + & & d & & + & + & [88] \\
\hline 4 & EHD2 & EH domain-containing protein 2 & + & & & & & & $\mathrm{u}$ & d & & & + & \\
\hline 3 & EIF2A & $\begin{array}{l}\text { Eukaryotic translation initiation factor } 2 \\
\text { subunit } 1 \text {, EIF2S1 }\end{array}$ & + & + & + & + & & & & & & & + & [89] \\
\hline 10 & EIF3A & $\begin{array}{l}\text { Eukaryotic translation initiation factor } 3 \\
\text { subunit } A\end{array}$ & + & + & & + & & & $\mathrm{u}$ & $d$ & Nsp1 & + & + & {$[90]$} \\
\hline 9 & EIF3B & $\begin{array}{l}\text { Eukaryotic translation initiation factor } 3 \\
\text { subunit B }\end{array}$ & + & + & & + & & & $\mathrm{u}$ & $d$ & Nsp1 & + & + & [39] \\
\hline 2 & EIF3C & $\begin{array}{l}\text { Eukaryotic translation initiation factor } 3 \\
\text { subunit C }\end{array}$ & & + & + & & & & & $d$ & Nsp1 & & + & {$[91]$} \\
\hline 3 & EIF3CL & $\begin{array}{l}\text { Eukaryotic translation initiation factor } 3 \\
\text { subunit C-like protein }\end{array}$ & + & & & + & & & & $d$ & & + & & \\
\hline 5 & EIF3E & $\begin{array}{l}\text { Eukaryotic translation initiation factor } 3 \\
\text { subunit } E\end{array}$ & + & + & + & + & & & $\mathrm{u}$ & $d$ & Nsp1 & + & + & [92] \\
\hline 4 & EIF3F & $\begin{array}{l}\text { Eukaryotic translation initiation factor } 3 \\
\text { subunit } F\end{array}$ & + & + & & & & & u & $d$ & Nsp1 & + & + & [93] \\
\hline 2 & EIF3G & $\begin{array}{l}\text { Eukaryotic translation initiation factor } 3 \\
\text { subunit } \mathrm{G}\end{array}$ & + & & & & & & & & Nsp1 & & + & [93] \\
\hline 2 & EIF3I & $\begin{array}{l}\text { Eukaryotic translation initiation factor } 3 \\
\text { subunit I }\end{array}$ & & + & & & & & & $d$ & Nsp1 & & + & [91] \\
\hline 10 & EIF3L & EIF3, subunit E interacting protein & + & + & + & + & & & & $d$ & Nsp1 & + & + & [39] \\
\hline 19 & EIF4A1 & Eukaryotic initiation factor $4 \mathrm{~A}-1, \mathrm{DDX} 2 \mathrm{~A}$ & + & + & & + & + & & u & d & & & + & \\
\hline 8 & EIF4A3 & Eukaryotic initiation factor 4A-III, DDX48 & + & + & & + & & & & & & + & + & {$[94]$} \\
\hline 4 & EIF4G1 & $\begin{array}{l}\text { Eukaryotic translation initiation factor } 4 \\
\text { gamma } 1\end{array}$ & + & & + & & & & $\mathrm{u}$ & d & & & + & [93] \\
\hline 2 & EIF4G2 & $\begin{array}{l}\text { Eukaryotic translation initiation factor } 4 \\
\text { gamma } 2\end{array}$ & + & & & & & & & d & Nsp1 & & + & [93] \\
\hline 2 & EIF5 & Eukaryotic translation initiation factor 5 & & + & & & & & $\mathrm{u}$ & d & & & + & [95] \\
\hline 5 & EIF5A & Eukaryotic translation initiation factor $5 \mathrm{~A}-1$ & + & + & & + & & & $\mathrm{u}$ & $d$ & & & + & [95] \\
\hline 2 & EIF5A2 & Eukaryotic translation initiation factor $5 \mathrm{~A}-2$ & + & & + & & + & & & d & & & + & [95] \\
\hline 2 & EIF5B & $\begin{array}{l}\text { Eukaryotic translation initiation factor } 5 b \\
\text { (eif-5b) (translation initiation factor if-2) }\end{array}$ & & & + & & & & $\mathrm{u}$ & & & & + & \\
\hline 3 & EIF6 & Eukaryotic translation initiation factor 6 & + & + & + & & & & $\mathrm{u}$ & & & & + & \\
\hline 4 & ELAVL1 & ELAV-like protein & + & + & & & & & & d & & & + & [96] \\
\hline 2 & ELOB & Transcription elongation factor B, TCEB2 & + & & & & & & $\mathrm{u}$ & d & $\begin{array}{l}\text { Nsp16 } \\
\text { Orf10 }\end{array}$ & & + & \\
\hline 2 & EMG1 & $\begin{array}{l}\text { Ribosomal RNA small subunit } \\
\text { methyltransferase NEP1 }\end{array}$ & & & & + & & & $\mathrm{u}$ & $\mathrm{d}$ & & & + & \\
\hline 12 & ENO1 & Alpha-enolase & + & & + & + & & + & $\mathrm{u}$ & $d$ & & & + & {$[97]$} \\
\hline 7 & ENO2 & Gamma-enolase & + & + & & & & & $\mathrm{u}$ & $d$ & & & + & [98] \\
\hline 2 & ENOPH1 & Enolase-phosphatase E1 & + & & & & & & $\mathrm{u}$ & & & & + & \\
\hline 6 & EPHX1 & Epoxide hydrolase & & & & + & & & & $d$ & & + & & {$[99]$} \\
\hline 4 & ERO1A & $\begin{array}{l}\text { Endoplasmic oxidoreductin-1-like protein, } \\
\text { ERO1L }\end{array}$ & & + & & & & + & & $d$ & Orf3a & & + & \\
\hline 6 & ERP44 & $\begin{array}{l}\text { Endoplasmic reticulum resident protein } \\
\text { ERp44 }\end{array}$ & + & & & & & & & & Orf8 & & + & [101] \\
\hline 4 & ESYT1 & Extended synaptotagmin-1, FAM62A & + & & & + & & & & & $\begin{array}{c}\mathrm{E} \\
\mathrm{M} \\
\text { Nsp3 } \\
\text { Nsp4 } \\
\text { Nsp6 } \\
\text { Orf3a } \\
\text { Orf6 } \\
\text { Orf7a } \\
\text { Orf7b } \\
\text { Orf8 } \\
\text { Orf9c }\end{array}$ & + & & [102] \\
\hline
\end{tabular}


bioRxiv preprint doi: https://doi.org/10.1101/2021.07.30.454526; this version posted August 4, 2021. The copyright holder for this preprint (which was not certified by peer review) is the author/funder, who has granted bioRxiv a license to display the preprint in perpetuity. It is made available under aCC-BY 4.0 International license.

\begin{tabular}{|c|c|c|c|c|c|c|c|c|c|c|c|c|c|c|}
\hline & & & & & & & & & & & S & & & \\
\hline 4 & ETF1 & $\begin{array}{l}\text { Eukaryotic peptide chain release factor } \\
\text { subunit } 1\end{array}$ & & + & & & & & $\mathrm{u}$ & & & & + & \\
\hline 2 & EWSR1 & EWS RNA-binding protein & + & & & & & & $\mathrm{u}$ & $d$ & & & + & \\
\hline 14 & EZR & Ezrin & & + & & + & & & $\mathrm{u}$ & $d$ & S & & + & [103] \\
\hline 2 & FAF1 & FAS-associated factor 1 & + & & & & & & $\mathrm{u}$ & & & & + & \\
\hline 3 & FARSB & Phenylalanine-tRNA ligase beta subunit & & + & & & & & & & & & + & [104] \\
\hline 19 & FASN & Fatty acid synthase & + & + & + & & + & & u & d & & & + & [105] \\
\hline 3 & FBLN1 & Fibulin 1 & + & & & & & & $\mathrm{u}$ & d & & & + & [106] \\
\hline 2 & FDPS & $\begin{array}{l}\text { Farnesyl pyrophosphate synthetase like- } 4 \\
\text { protein }\end{array}$ & & + & & & & & & d & & & + & \\
\hline 2 & FEN1 & Flap endonuclease 1 & & + & & & & & $\mathrm{u}$ & d & & & + & \\
\hline 2 & FERMT3 & Fermitin family homolog 3 & & + & & & & & $\mathrm{u}$ & & & & + & \\
\hline 8 & FKBP10 & FK506-binding protein 10 & + & & & & & & & & Orf8 & & + & \\
\hline 11 & FKBP4 & $\begin{array}{l}\text { Peptidyl-prolyl cis-trans isomerase FKBP4, } \\
\text { FKBP-52 }\end{array}$ & & + & & + & & & & & Nsp12 & & + & [107] \\
\hline 2 & FKBP5 & $\begin{array}{l}\text { Peptidyl-prolyl cis-trans isomerase FKBP5 } \\
\text { (FK506-binding protein) }\end{array}$ & & + & & & & & $\mathrm{u}$ & & & & + & \\
\hline 4 & FKBP9 & FK506-binding protein 9 & + & & & & & & & $d$ & & & + & \\
\hline 43 & FLNA & Filamin-A & + & & & + & & + & $\mathrm{u}$ & d & & + & + & [108] \\
\hline 25 & FLNB & Filamin-B & + & & & + & & + & $\mathrm{u}$ & & & & + & {$[57]$} \\
\hline 24 & FLNC & Filamin-C & + & & & + & & & $\mathrm{u}$ & $d$ & & + & + & [109] \\
\hline 23 & FN1 & Fibronectin & + & & & & & & $\mathrm{u}$ & $d$ & & & + & [110] \\
\hline 3 & FSTL1 & Follistatin-related protein & + & & & & & & $\mathrm{u}$ & $d$ & & & + & [111] \\
\hline 2 & FTH1 & Ferritin heavy chain & + & & & + & & & $\mathrm{u}$ & $d$ & & & + & [111] \\
\hline 2 & FUBP1 & Far upstream element-binding protein 1 & & + & & & & & $\mathrm{u}$ & d & & & + & [112] \\
\hline 10 & G6PD & Glucose-6-phosphate 1-dehydrogenase & + & + & & + & & & $\mathrm{u}$ & d & & + & + & {$[44]$} \\
\hline 15 & GANAB & Neutral alpha-glucosidase $A B$ & + & + & & + & & & & $d$ & $\begin{array}{l}\text { Orf6 } \\
\text { Orf8 } \\
\text { Orf9c }\end{array}$ & & + & [113] \\
\hline 6 & GAPDH & $\begin{array}{l}\text { Glyceraldehyde-3-phosphate } \\
\text { dehydrogenase }\end{array}$ & + & + & + & + & & & $\mathrm{u}$ & $\mathrm{d}$ & Orf8 & + & + & [114] \\
\hline 2 & GAR1 & $\begin{array}{l}\text { H/ACA ribonucleoprotein complex subunit } \\
1\end{array}$ & + & & & + & & & & & & + & & \\
\hline 4 & GARS & Glycine-tRNA ligase, GARS1 & & + & & & & & $\mathrm{u}$ & & & & + & [115] \\
\hline 2 & GART & $\begin{array}{l}\text { Trifunctional purine biosynthetic protein } \\
\text { adenosine-3 }\end{array}$ & & + & & & & & & $d$ & Nsp15 & & + & \\
\hline 2 & GBE1 & 1,4-alpha-glucan-branching enzyme & & + & & + & & & u & & & & + & \\
\hline 4 & GCLC & Glutamate-cysteine ligase catalytic subunit & & & & + & & & & & Orf3 & & + & \\
\hline 8 & GDI1 & Rab GDP dissociation inhibitor alpha & + & + & & + & & & $\mathrm{u}$ & d & & & + & [116] \\
\hline 10 & GDI2 & Rab GDP dissociation inhibitor beta & + & + & & + & & & $\mathrm{u}$ & $\mathrm{d}$ & $\begin{array}{l}\text { Nsp12 } \\
\text { Orf9b }\end{array}$ & & + & [117] \\
\hline 2 & GGCT & $\begin{array}{l}\text { Gamma-glutamylcyclotransferase, } \\
\text { cytochrome c-releasing factor } 21\end{array}$ & & + & & & & & u & & & & + & \\
\hline 3 & GLO1 & Lactoylglutathione lyase & & + & & + & & & & $d$ & Orf3 & & + & [118] \\
\hline 3 & GLRX3 & Glutaredoxin 3, Thioredoxin-like 2 & + & + & & & & & & $d$ & & & + & [119] \\
\hline 10 & GLUD1 & $\begin{array}{l}\text { Glutamate dehydrogenase } 1 \\
\text { mitochondrial }\end{array}$ & & + & & & & & & & & & + & [120] \\
\hline 2 & GMFB & Glia maturation factor, beta & + & + & & & & & $\mathrm{u}$ & & & & + & \\
\hline 2 & GPALPP1 & $\begin{array}{l}\text { Lipopolysaccharide-specific response } \\
\text { protein } 7\end{array}$ & & & + & & & & & & & & + & \\
\hline 5 & GPC1 & Glypican-1 & + & & & + & & & & d & & + & & \\
\hline 2 & GPI & Glucose-6-phosphate isomerase & & & & + & & & u & $d$ & $\begin{array}{c}E \\
\text { Nsp6 } \\
\text { Orf3 } \\
\text { Orf3b } \\
\text { Orf6 }\end{array}$ & & + & [121] \\
\hline 4 & GRWD1 & $\begin{array}{l}\text { Glutamate-rich WD repeat-containing } \\
\text { protein } 1\end{array}$ & & & & + & & & & & & + & & \\
\hline 16 & GSN & Gelsolin & + & & & & & & $\mathrm{u}$ & $d$ & & & + & {$[16]$} \\
\hline 3 & GSPT1 & $\begin{array}{l}\text { Eukaryotic peptide chain release factor } \\
\text { GTP-binding subunit ERF3A }\end{array}$ & & + & & & & & & & & & + & \\
\hline 3 & GSS & Glutathione synthetase & & + & & & & & & d & & & + & \\
\hline 6 & GSTP1 & Glutathione S-transferase & & & & + & & & $\mathrm{u}$ & $d$ & & & + & [122] \\
\hline 4 & GTF2I & General transcription factor II-I & + & + & & & & & u & d & & & + & [25] \\
\hline 3 & $\mathrm{H} 1-1$ & Histone H1.1, H1F1, HIST1H1A, H1FNT & & & + & & + & & $\mathrm{u}$ & d & & + & + & [123] \\
\hline 2 & H1F0 & Histone H1.0, H1FV, H1-0 & & & & & & + & $\mathrm{u}$ & d & Nsp3 & & + & \\
\hline
\end{tabular}


bioRxiv preprint doi: https://doi.org/10.1101/2021.07,30.454526. this version posted August 4, 2021. The copyright holder for this preprint (which was not certified by peer review) is the author/funder, who has granted bioRxiv a license to display the preprint in perpetuity. It is made available under aCC-BY 4.0 International license.

\begin{tabular}{|c|c|c|c|c|c|c|c|c|c|c|c|c|c|c|}
\hline & & & & & & & & & & & $\begin{array}{l}\text { Nsp8 } \\
\text { Orf3b } \\
\text { Orf10 }\end{array}$ & & & \\
\hline 3 & H2AFV & Histone H2A.V, H2AZ2 & + & + & & + & & + & $\mathrm{u}$ & d & & + & + & [127] \\
\hline 11 & H2AFY & Core histone macro-H2A.1, MACROH2A1 & & & & + & & + & $\mathrm{u}$ & & & + & & [128] \\
\hline 4 & H2AFY2 & Cor2 histone macro-H2A.2, MACROH2A2 & + & + & & + & & & $\mathrm{u}$ & & & + & & [128] \\
\hline 4 & HADHA & $\begin{array}{l}\text { Trifunctional enzyme subunit alpha, } \\
\text { mitochondrial }\end{array}$ & & & & + & & & & & & + & & \\
\hline 3 & HARS & Histidyl-tRNA synthetase, cytoplasmic & + & + & & + & & & & & & & + & [41] \\
\hline 5 & HDGF & Hepatoma-derived growth factor, HMG1L2 & + & + & + & & + & + & u & d & & & + & [134] \\
\hline 2 & HDLBP & $\begin{array}{l}\text { Vigilin, High density lipoprotein binding } \\
\text { protein }\end{array}$ & + & & & & & & $\mathrm{u}$ & $d$ & $\begin{array}{c}\mathrm{N} \\
\text { Nsp2 }\end{array}$ & & + & \\
\hline 4 & HEATR1 & HEAT repeat-containing protein 1 & & & & + & & & u & d & & + & & \\
\hline 2 & HEBP2 & Heme-binding protein 2 & + & & & & & & $\mathrm{u}$ & & & & + & \\
\hline 5 & HEXB & Beta-hexosaminidase subunit beta & + & & & & & & & d & & & + & \\
\hline 6 & HIST1H1B & Histone H1.5, H1F5, H1-5 & + & & + & + & + & + & $\mathrm{u}$ & d & & + & + & [124] \\
\hline 6 & HIST1H1C & Histone H1.2, H1F2, H1-2 & + & + & + & + & + & + & $\mathrm{u}$ & d & Nsp8 & + & + & [124] \\
\hline 4 & $\begin{array}{l}\text { HIST1H2A } \\
\text { A }\end{array}$ & Histone H2A type 1-A, H2AFR, H2AC1 & & & + & + & + & & & & & + & + & [125] \\
\hline 2 & $\begin{array}{l}\mathrm{HIST} 1 \mathrm{H} 2 \mathrm{~A} \\
\mathrm{~B}\end{array}$ & Histone H2A type 1-B/E, H2AFM, H2AC4 & & + & & & & & & $d$ & & + & & [126] \\
\hline 5 & $\begin{array}{l}\text { HIST1H2B } \\
\text { A }\end{array}$ & Histone H2B type 1-A, H2BC1 & & & + & & + & & & & & + & + & [123] \\
\hline 5 & $\begin{array}{l}\text { HIST1H2B } \\
\text { B }\end{array}$ & Histone $\mathrm{H} 2 \mathrm{~B}$ type 1-B, H2BFF, H2BC3 & & & + & & + & & & & & + & & [131] \\
\hline 2 & $\begin{array}{l}\mathrm{HIST} 1 \mathrm{H} 2 \mathrm{~B} \\
\mathrm{~L}\end{array}$ & Histone $\mathrm{H} 2 \mathrm{~B}$ type $1-\mathrm{L}, \mathrm{H} 2 \mathrm{BFC}, \mathrm{H} 2 \mathrm{BC} 13$ & + & + & & + & & & $\mathrm{u}$ & $d$ & & + & & [129] \\
\hline 12 & $\begin{array}{l}\text { HIST2H2B } \\
\text { E }\end{array}$ & Histone H2B type 2-E, H2BC21 & + & + & & + & & + & $\mathrm{u}$ & $d$ & & + & & [130] \\
\hline 5 & HIST2H3A & Histone H3.2, H3C15 & + & + & & + & & + & $\mathrm{u}$ & $d$ & & + & + & [132] \\
\hline 4 & HIST3H3 & Histone H3.1t, H3FT, H3-4 & & & + & & + & & & & & + & + & [123] \\
\hline 14 & HIST4H4 & Histone $\mathrm{H} 4, \mathrm{H} 4 \mathrm{C} 1$ & + & + & + & + & + & + & $\mathrm{u}$ & $d$ & & + & + & [133] \\
\hline 10 & HMGB1 & High mobility group protein 1 & & + & + & & + & & & $d$ & & & + & [135] \\
\hline 3 & HMGCS1 & $\begin{array}{l}\text { Hydroxymethylglutaryl-CoA synthase, } \\
\text { cytoplasmic }\end{array}$ & & + & + & & + & & $\mathrm{u}$ & $d$ & & & + & \\
\hline 2 & HMGN1 & Non-histone chromosomal protein HMG14 & & & + & & & & $\mathrm{u}$ & & & & + & \\
\hline 4 & HNRNPA1 & $\begin{array}{l}\text { Heterogeneous nuclear ribonucleoprotein } \\
\text { A1 }\end{array}$ & + & + & + & & + & + & $\mathrm{u}$ & $d$ & & & + & [137] \\
\hline 8 & $\begin{array}{l}\text { HNRNPA2 } \\
\text { B1 }\end{array}$ & hnRNP A2/B1 & + & + & + & + & & + & $\mathrm{u}$ & $d$ & & & + & [138] \\
\hline 2 & HNRNPA3 & hnRNP A3 & + & + & & & & + & $\mathrm{u}$ & $d$ & & & + & [139] \\
\hline 2 & HNRNPAB & hnRNP A/B & & + & & & & & & $d$ & & & + & [139] \\
\hline 3 & HNRNPC & hnRNP C1/C2 & + & + & + & + & & & $\mathrm{u}$ & $d$ & & + & + & [140] \\
\hline 7 & $\begin{array}{l}\text { HNRNPCL } \\
1\end{array}$ & hnRNP C-like 1 & + & + & + & + & + & + & & & & + & + & [141] \\
\hline 5 & HNRNPD & hnRNP D, AUF1 & + & + & + & & & & & & & & + & [142] \\
\hline 3 & HNRNPDL & hnRNP D-like & + & + & & & & & $\mathrm{u}$ & $d$ & & & + & [143] \\
\hline 5 & HNRNPF & hnRNP F & + & + & & + & & & & $d$ & & + & + & [144] \\
\hline 2 & HNRNPH1 & hnRNP H1 & + & + & + & + & & & $\mathrm{u}$ & $d$ & & & + & \\
\hline 2 & HNRNPH3 & hnRNP H3 & + & & & & & & $\mathrm{u}$ & $d$ & & & + & [145] \\
\hline 9 & HNRNPK & hnRNP K & + & + & + & + & + & & $\mathrm{u}$ & & & & + & [146] \\
\hline 3 & HNRNPM & hnRNP M & & & & + & & & $\mathrm{u}$ & $d$ & & + & & \\
\hline 6 & HNRNPQ & hnRNP Q, SYNCRIP & + & + & + & + & + & + & & $d$ & & & + & \\
\hline 7 & HNRNPR & hnRNP R & + & + & + & + & & + & u & $d$ & & & + & [147] \\
\hline 5 & HNRNPU & hnRNP U (scaffold attachment factor A) & + & + & + & + & + & + & $\mathrm{u}$ & $d$ & & + & + & [148] \\
\hline 6 & $\begin{array}{l}\text { HNRNPUL } \\
1\end{array}$ & hnRNP U-like protein 1 & + & & & + & & & $\mathrm{u}$ & $d$ & & + & & \\
\hline 4 & $\begin{array}{l}\text { HNRNPUL } \\
2\end{array}$ & hnRNP U-like protein 2 & & & & + & & & $\mathrm{u}$ & $d$ & & + & & \\
\hline 6 & HPRT1 & $\begin{array}{l}\text { Hypoxanthine-guanine } \\
\text { phosphoribosyltransferase }\end{array}$ & & + & & & & & & & & & + & \\
\hline 2 & HSP70B & $\begin{array}{l}\text { Putative heat shock } 70 \mathrm{kDa} \text { protein, } \\
\text { HSPA7 }\end{array}$ & & & & + & & & $\mathrm{u}$ & $d$ & & + & & \\
\hline 38 & HSP90AA1 & Heat shock protein 90 -alpha & + & + & + & + & + & + & $\mathrm{u}$ & d & & + & + & [149] \\
\hline 6 & HSP90AA2 & Heat shock protein 90 -alpha A2 & + & + & & + & & + & $\mathrm{u}$ & & & + & + & [150] \\
\hline 16 & HSP90AB1 & Heat shock protein HSP 90-beta & + & + & + & + & + & + & $\mathrm{u}$ & $d$ & Nsp12 & & + & [151] \\
\hline 31 & HSP90B1 & Endoplasmin, GRP94 & + & + & + & + & + & + & $\mathrm{u}$ & d & Orf3a & + & + & [152] \\
\hline
\end{tabular}


bioRxiv preprint doi: https://doi.org/10.1101/2021.07,30.454526. this version posted August 4, 2021. The copyright holder for this preprint (which was not certified by peer review) is the author/funder, who has granted bioRxiv a license to display the preprint in perpetuity. It is made available under aCC-BY 4.0 International license.

\begin{tabular}{|c|c|c|c|c|c|c|c|c|c|c|c|c|c|c|}
\hline & & & & & & & & & & & Orf9c & & & \\
\hline 7 & HSPA1A & Heat shock $70 \mathrm{kDa}$ protein $1 \mathrm{~A}$ & + & & & + & & & u & $d$ & $\begin{array}{c}\mathrm{N} \\
\text { Orf9b }\end{array}$ & & + & \\
\hline 4 & HSPA1L & Heat shock 70 kDa protein 1-like, HSP70T & + & & & + & & & & & & & + & [153] \\
\hline 2 & HSPA2 & Heat shock 70 kda protein 2 & & & + & & & & $\mathrm{u}$ & & Nsp3 & + & & \\
\hline 14 & HSPA4 & Heat shock $70 \mathrm{kDa}$ protein 4 & + & + & + & & + & & $\mathrm{u}$ & $d$ & & & + & {$[154]$} \\
\hline 35 & HSPA5 & $\begin{array}{l}\text { Endoplasmic reticulum chaperone BiP, } \\
\text { GRP78 }\end{array}$ & + & + & + & + & + & + & $\mathrm{u}$ & $\mathrm{d}$ & $\begin{array}{c}\mathrm{E} \\
\mathrm{M} \\
\text { Nsp2 } \\
\text { Nsp4 } \\
\text { Nsp6 } \\
\text { Orf3a } \\
\text { Orf7a } \\
\text { Orf7b } \\
\text { S }\end{array}$ & & + & [155] \\
\hline 27 & HSPA8 & Heat shock cognate $71 \mathrm{kDa}$ protein & + & + & + & + & & + & $\mathrm{u}$ & $d$ & $\begin{array}{l}\text { Nsp2 } \\
\text { Nsp12 }\end{array}$ & & + & {$[156]$} \\
\hline 25 & HSPA9 & Stress-70 protein, mitochondrial (GRP75) & + & + & + & + & & + & $u$ & $d$ & $\mathrm{~N}$ & & + & [156] \\
\hline 7 & HSPB1 & Heat shock protein beta-1 & + & & & & & & $\mathrm{u}$ & $d$ & & & + & [157] \\
\hline 2 & HSPBP1 & Hsp70-binding protein 1 & & & & + & & & $\mathrm{u}$ & $d$ & S & & + & \\
\hline 30 & HSPD1 & $60 \mathrm{kDa}$ heat shock protein, mitochondrial & + & + & + & & + & & $\mathrm{u}$ & $d$ & & & + & [158] \\
\hline 3 & HSPG2 & $\begin{array}{l}\text { Basement membrane heparan sulfate } \\
\text { proteoglycan }\end{array}$ & + & & & + & & & $\mathrm{u}$ & $d$ & & + & & [159] \\
\hline 13 & HSPH1 & Heat shock protein $105 \mathrm{kDa}$ & & + & + & & + & & u & & & & + & [160] \\
\hline 4 & HTATSF1 & HIV Tat-specific factor 1 & + & & & + & & & & $\mathrm{d}$ & & + & & \\
\hline 7 & HYOU1 & Hypoxia up-regulated protein & + & & + & & + & & $\mathrm{u}$ & & $\begin{array}{l}\text { Nsp4 } \\
\text { Orf3a } \\
\text { Orf8 }\end{array}$ & & + & {$[161]$} \\
\hline 4 & IDE & Insulin-degrading enzyme & & + & & + & & & & & $\begin{array}{c}\text { Nsp4 } \\
\text { Nsp12 } \\
\text { Nsp14 } \\
\text { Nsp15 } \\
\text { Nsp16 } \\
\text { Orf3b }\end{array}$ & & + & \\
\hline 2 & IDH3A & $\begin{array}{l}\text { Isocitrate dehydrogenase [NAD] subunit } \\
\text { alpha, mitochondrial }\end{array}$ & & + & & & & & & & & & + & \\
\hline 2 & IGBP1 & Immunoglobulin-binding protein 1 & + & & & & & & u & $\mathrm{d}$ & & & + & \\
\hline 2 & IL18 & Interleukin-18 & & & & + & & & u & $\mathrm{d}$ & & & + & [162] \\
\hline 7 & ILF2 & Interleukin enhancer-binding factor 2 & + & + & + & + & & & u & & & + & + & [163] \\
\hline 6 & ILF3 & Interleukin enhancer-binding factor 3 & + & & + & & & & $\mathrm{u}$ & & & & + & [163] \\
\hline 2 & IMPDH2 & $\begin{array}{l}\text { Inosine-5'-monophosphate dehydrogenase } \\
2 \text { (imp dehydrogenase 2) (impdh-ii) }\end{array}$ & & & + & & & & & $\mathrm{d}$ & Nsp14 & & + & \\
\hline 7 & IPO5 & Importin-5, KPNB3, RANBP5 & & + & + & & + & & & & & & + & [164] \\
\hline 3 & IPO7 & Importin-7, RANBP7 & & + & & & & & & & $\begin{array}{l}\text { Nsp6 } \\
\text { Orf9c }\end{array}$ & & + & {$[165]$} \\
\hline 13 & IQGAP1 & $\begin{array}{l}\text { Ras GTPase-activating-like protein } \\
\text { IQGAP1 }\end{array}$ & + & + & & + & & + & $\mathrm{u}$ & & & + & + & [166] \\
\hline 2 & IRGQ & Immunity-related GTPase family Q protein & + & & & & & & $\mathrm{u}$ & $d$ & & & + & \\
\hline 4 & ITGB1 & Integrin beta-1 & + & & & + & & & $\mathrm{u}$ & $d$ & $\begin{array}{l}\text { Nsp4 } \\
\text { Of3b } \\
\text { Orf6 } \\
\text { Orf8 } \\
\text { Orf9c }\end{array}$ & + & & [167] \\
\hline 2 & IWS1 & Protein IWS1 homolog & & & & + & & & $\mathrm{u}$ & d & & + & & \\
\hline 4 & KARS & Lysyl-tRNA synthetase & + & + & & + & & & & & Nsp7 & + & + & {$[100]$} \\
\hline 3 & KHSRP & $\begin{array}{l}\text { Far upstream element-binding protein } 2 \\
\text { (KH-type splicing regulatory protein), } \\
\text { FUBP2 }\end{array}$ & & + & & & & & $\mathrm{u}$ & $d$ & & & + & [53] \\
\hline 2 & KPNA2 & Importin subunit alpha-1 & & + & & & & & & $\mathrm{d}$ & Orf6 & & + & \\
\hline 2 & KPNA3 & Importin subunit alpha-4 & + & & + & & & & & & & + & + & \\
\hline 11 & KPNB1 & Importin subunit beta-1 & + & + & + & + & + & + & & & & + & + & {$[164]$} \\
\hline 2 & KRR1 & $\begin{array}{l}\text { KRR1 small subunit processome } \\
\text { component homolog, HIV-1 Rev-binding } \\
\text { protein }\end{array}$ & & & & + & & & & $d$ & & + & & [168] \\
\hline 10 & KTN1 & Kinectin & + & & & & & & u & & Orf6 & & + & [169] \\
\hline 2 & KYNU & Kynureninase & & & & + & & & $\mathrm{u}$ & & Orf3 & & + & \\
\hline 7 & LAMB1 & Laminin subunit beta-1 & + & & & & & & & $d$ & & & + & {$[170]$} \\
\hline 5 & LAMC1 & Laminin subunit gamma-1 & + & & & & & & $\mathrm{u}$ & $d$ & & & + & {$[171]$} \\
\hline
\end{tabular}


bioRxiv preprint doi: https://doi.org/10.1101/2021.07 30.454526; this version posted Auqust 4, 2021. The copyright holder for this preprint (which was not certified by peer review) is the author/funder, who has granted bioRxiv a license to display the preprint in perpetuity. It is made available under aCC-BY 4.0 International license.

\begin{tabular}{|c|c|c|c|c|c|c|c|c|c|c|c|c|c|c|}
\hline 2 & LAMP2 & $\begin{array}{l}\text { Lysosome-associated membrane } \\
\text { glycoprotein } 2\end{array}$ & & & & + & & & $\mathrm{u}$ & d & & & + & [172] \\
\hline 2 & LARS & Leucyl-tRNA synthetase, cytoplasmic & & & & + & & & & & & + & & {$[100]$} \\
\hline 8 & LDHA & L-lactate dehydrogenase A chain & & + & + & + & & & $\mathrm{u}$ & $d$ & Nsp12 & & + & [173] \\
\hline 10 & LDHB & L-lactate dehydrogenase $\mathrm{B}$ chain & & + & + & + & & & $\mathrm{u}$ & $d$ & $\begin{array}{l}\text { Nsp12 } \\
\text { Nsp7 }\end{array}$ & & + & {$[174]$} \\
\hline 2 & LEO1 & RNA polymerase-associated protein LEO1 & & & & + & & & $\mathrm{u}$ & & & + & & \\
\hline 5 & LGALS1 & Galectin-1 & + & & & & & & $\mathrm{u}$ & d & & & + & {$[175]$} \\
\hline 23 & LMNA & Prelamin-A/C & + & & & + & & + & $\mathrm{u}$ & d & $\begin{array}{l}\text { Nsp13 } \\
\text { Orf3b } \\
\text { Orf8 } \\
\text { Orf10 }\end{array}$ & & + & {$[176]$} \\
\hline 8 & LMNB1 & Lamin-B1 & + & & + & & + & & $\mathrm{u}$ & d & & + & + & {$[177]$} \\
\hline 7 & LMNB2 & Lamin-B2 & + & & & + & & & $\mathrm{u}$ & d & & + & & [178] \\
\hline 16 & LRPPRC & Leucine-rich PPR motif-containing protein & + & + & + & + & & + & & d & & & + & [179] \\
\hline 2 & LSM2 & $\begin{array}{l}\text { U6 snRNA-associated Sm-like protein } \\
\text { LSm2 }\end{array}$ & + & & & & & & $\mathrm{u}$ & & & & + & \\
\hline 2 & LSM6 & $\begin{array}{l}\text { U6 snRNA-associated Sm-like protein } \\
\text { LSm6 }\end{array}$ & + & & & & & & $\mathrm{u}$ & & & & + & \\
\hline 2 & LSM8 & $\begin{array}{l}\text { U6 snRNA-associated Sm-like protein } \\
\text { LSm8 }\end{array}$ & & & & & + & & & & & & + & \\
\hline 2 & MAGOHB & Protein mago nashi homolog & + & & & & & & $\mathrm{u}$ & d & & & + & \\
\hline 3 & MANBA & Beta-mannosidase & + & & & & & & & d & & & + & \\
\hline 3 & MAP1B & Microtubule-associated protein 1B & + & & & + & & & $\mathrm{u}$ & $d$ & & + & + & {$[180]$} \\
\hline 6 & MAPRE1 & $\begin{array}{l}\text { Microtubule-associated protein RP/EB } \\
\text { family member }\end{array}$ & + & & + & & + & & & & Orf3 & & + & \\
\hline 2 & MARCKS & $\begin{array}{l}\text { Myristoylated alanine-rich c-kinase } \\
\text { substrate (marcks) (protein kinase c } \\
\text { substrate, } 80 \text { kda protein, light chain) } \\
\text { (pkcsl) }\end{array}$ & & & + & & & & $\mathrm{u}$ & $d$ & $\begin{array}{c}\text { M } \\
\text { Nsp4 } \\
\text { Nsp6 } \\
\text { Orf3a } \\
\text { Orf3b } \\
\text { Orf7b } \\
\text { S }\end{array}$ & & + & \\
\hline 2 & MARS & Methionine-tRNA ligase, MARS1 & & & & + & & & & d & & + & & [39] \\
\hline 9 & MCM2 & DNA replication licensing factor MCM2 & & + & + & & + & + & & $\mathrm{d}$ & & & + & [181] \\
\hline 7 & MCM3 & DNA replication licensing factor $\mathrm{MCM} 3$ & & + & + & & + & + & $\mathrm{u}$ & d & & & + & [39] \\
\hline 5 & MCM4 & DNA replication licensing factor MCM4 & & + & + & & + & & $\mathrm{u}$ & $\mathrm{d}$ & & & + & {$[181]$} \\
\hline 3 & MCM5 & DNA replication licensing factor MCM5 & & & + & & + & & $\mathrm{u}$ & d & & & + & {$[181]$} \\
\hline 9 & MCM6 & DNA replication licensing factor MCM6 & & + & + & & + & + & $\mathrm{u}$ & $d$ & & & + & [39] \\
\hline 2 & $\mathrm{MDH} 1$ & Malate dehydrogenase, cytoplasmic & & & + & & & & & $\mathrm{d}$ & $\begin{array}{c}E \\
\text { Orf3 }\end{array}$ & & + & \\
\hline 3 & $\mathrm{MDH} 2$ & Malate dehydrogenase, mitochondrial & & & & + & & & $\mathrm{u}$ & $d$ & & & + & [25] \\
\hline 2 & ME2 & $\begin{array}{l}\text { NAD-dependent malic enzyme, } \\
\text { mitochondrial }\end{array}$ & & + & & & & & $\mathrm{u}$ & $d$ & Nsp15 & & + & \\
\hline 10 & MOV10 & $\begin{array}{l}\text { Putative helicase, Moloney leukemia virus } \\
10 \text { protein }\end{array}$ & + & & & + & & & $\mathrm{u}$ & $\mathrm{d}$ & $\begin{array}{c}E \\
M \\
N \\
\text { Nsp3 } \\
\text { Nsp4 } \\
\text { Nsp6 } \\
\text { Orf3a } \\
\text { Orf7a } \\
\text { Orf7b } \\
\text { Orf8 } \\
\text { Orf9c } \\
\text { S }\end{array}$ & + & & \\
\hline 5 & MRPL1 & 39S ribosomal protein L1, mitochondrial & & & & + & & & & & & + & & \\
\hline 3 & MRPL13 & 39S ribosomal protein L13, mitochondrial & & & & + & & & & d & & + & & \\
\hline 2 & MRPL15 & 39S ribosomal protein L15, mitochondrial & & & & + & & & $\mathrm{u}$ & d & & + & & \\
\hline 2 & MRPL17 & 39S ribosomal protein L17, mitochondrial & & & & + & & & & $d$ & & + & & \\
\hline 2 & MRPL18 & 39S ribosomal protein L18, mitochondrial & & & & + & & & & $\mathrm{d}$ & & + & & \\
\hline 4 & MRPL19 & 39S ribosomal protein L19, mitochondrial & & & & + & & & & $d$ & Orf8 & + & & \\
\hline 2 & MRPL2 & 39S ribosomal protein L2, mitochondrial & & & & + & & & & d & Nsp6 & + & & \\
\hline 2 & MRPL23 & 39S ribosomal protein L23, mitochondrial & & & & + & & & & $\mathrm{d}$ & & + & & \\
\hline 5 & MRPL37 & 39S ribosomal protein L37, mitochondrial & & & & + & & & $\mathrm{u}$ & d & & + & & \\
\hline 5 & MRPL38 & 39S ribosomal protein L38, mitochondrial & & & & + & & & & $d$ & & + & & \\
\hline 2 & MRPL39 & 39S ribosomal protein L39, mitochondrial & & & & + & & + & & $\mathrm{d}$ & & + & + & \\
\hline
\end{tabular}


bioRxiv preprint doi: https://doi.org/10.1101/2021.07.30.454526; this version posted August 4, 2021. The copyright holder for this preprint (which was not certified by peer review) is the author/funder, who has granted bioRxiv a license to display the preprint in perpetuity. It is made available under aCC-BY 4.0 International license.

\begin{tabular}{|c|c|c|c|c|c|c|c|c|c|c|c|c|c|c|}
\hline 3 & MRPL45 & 39S ribosomal protein L45, mitochondrial & & & & + & & & & $d$ & & + & & \\
\hline 2 & MRPL49 & 39S ribosomal protein L49, mitochondrial & & & & + & & & & d & & + & & \\
\hline 4 & MRPS22 & $28 \mathrm{~S}$ ribosomal protein $\mathrm{S} 22$, mitochondrial & & & & + & & & & & & + & & \\
\hline 4 & MRPS23 & $28 \mathrm{~S}$ ribosomal protein S23, mitochondrial & & & & + & & & & & & + & & \\
\hline 6 & MRPS27 & $28 \mathrm{~S}$ ribosomal protein $\mathrm{S} 27$, mitochondrial & & & & + & & & & & Nsp8 & + & & \\
\hline 2 & MRPS28 & $\begin{array}{l}28 S \text { ribosomal protein S28, mitochondrial, } \\
\text { MRPS } 35\end{array}$ & & & & + & & & & & & + & & \\
\hline 2 & MRPS30 & $28 \mathrm{~S}$ ribosomal protein $\mathrm{S} 30$, mitochondrial & & & & + & & & & $\mathrm{d}$ & & + & & \\
\hline 2 & MRPS34 & $28 \mathrm{~S}$ ribosomal protein S34, mitochondrial & & & & + & & & & d & & + & & \\
\hline 3 & MRPS9 & 28S ribosomal protein S9, mitochondrial & & & & + & & & & & & + & & \\
\hline 6 & MSN & Moesin & + & + & & + & & & $\mathrm{u}$ & & $\begin{array}{l}\text { Nsp6 } \\
\text { Orf3 } \\
\text { S }\end{array}$ & & + & [182] \\
\hline 21 & MVP & Major vault protein & + & & & + & & & $\mathrm{u}$ & d & & + & & [183] \\
\hline 4 & MXRA5 & Matrix-remodeling-associated protein 5 & + & & & & & & & $d$ & & + & & [183] \\
\hline 16 & MYBBP1A & Myb-binding protein $1 \mathrm{~A}$ & & & + & + & & + & $\mathrm{u}$ & d & & + & + & \\
\hline 2 & MYG1 & $\begin{array}{l}\text { UPF0160 protein MYG1, mitochondrial, } \\
\text { C12orf10 }\end{array}$ & & + & & + & & & & & & & + & \\
\hline 2 & MYH10 & Myosin-10 & + & & & & & & $\mathrm{u}$ & $d$ & Nsp9 & + & & [184] \\
\hline 43 & MYH9 & Myosin-9 & + & & + & + & & + & $\mathrm{u}$ & d & & + & + & [184] \\
\hline 3 & MYL6 & Myosin light chain 6 & + & & & & + & & $\mathrm{u}$ & & & & + & [185] \\
\hline 4 & MYLK & Myosin light chain kinase, smooth muscle & + & & & & & & $\mathrm{u}$ & d & & & + & \\
\hline 3 & MYO1C & Unconventional myosin-lc, MYO1E & + & & & + & & & $\mathrm{u}$ & d & & + & & [186] \\
\hline 4 & MZB1 & $\begin{array}{l}\text { Marginal zone B- and B1-cell-specifc } \\
\text { protein (Proapoptotic caspase adapter } \\
\text { protein, plasma cell-induced resident } \\
\text { protein) }\end{array}$ & & + & & & & & $\mathrm{u}$ & & & & + & \\
\hline 3 & NAA15 & $\begin{array}{l}\mathrm{N} \text {-alpha-acetyltransferase } 15, \text { NatA } \\
\text { auxiliary subunit (NMDA receptor- } \\
\text { regulated protein, NARG1) }\end{array}$ & & + & & & & & & $d$ & & & + & \\
\hline 2 & NAA25 & $\begin{array}{l}\mathrm{N} \text {-alpha-acetyltransferase } 25 \text {, NatB } \\
\text { auxiliary subunit (TPR repeat-containing } \\
\text { protein } \mathrm{C} 12 \text { orf30) }\end{array}$ & & + & & & & & & & & & + & \\
\hline 4 & NACA & $\begin{array}{l}\text { Nascent polypeptide associated complex } \\
\text { subunit alpha }\end{array}$ & + & + & + & & + & & $\mathrm{u}$ & d & & & + & [187] \\
\hline 7 & NAP1L1 & Nucleosome assembly protein 1 -like 1 & + & + & + & + & & & $\mathrm{u}$ & $d$ & & + & + & \\
\hline 7 & NAP1L4 & Nucleosome assembly protein 1 -like 4 & + & + & + & & & & $\mathrm{u}$ & $\mathrm{d}$ & & + & + & \\
\hline 5 & NARS & $\begin{array}{l}\text { Asparagine-tRNA ligase, cytoplasmic, } \\
\text { NARS1 }\end{array}$ & & + & & & & & & $d$ & & & + & [188] \\
\hline 6 & NASP & Nuclear autoantigenic sperm protein & + & + & + & & + & & $\mathrm{u}$ & d & & & + & [189] \\
\hline 23 & NCL & Nucleolin & + & + & + & + & + & + & $\mathrm{u}$ & $d$ & & + & + & [190] \\
\hline 2 & NES & Nestin & + & & & & & & $\mathrm{u}$ & $d$ & & & + & \\
\hline 2 & NEU1 & Sialidase-1 & + & & & & & & $\mathrm{u}$ & d & Orf8 & & + & [191] \\
\hline 3 & NEXN & Nexilin F-actin binding protein & + & & & & & & $\mathrm{u}$ & $d$ & & & + & \\
\hline 2 & NFU1 & HIRA interacting protein 5 & + & & & & & & & & & & + & \\
\hline 8 & NME1 & Nucleoside diphosphate kinase A, RMRP & + & + & & + & & & $\mathrm{u}$ & d & & & + & [192] \\
\hline 3 & NME2 & Nucleoside diphosphate kinase 2, NM23 & & & & + & & & $\mathrm{u}$ & $d$ & & & + & [193] \\
\hline 2 & NMT1 & $\begin{array}{l}\text { Glycylpeptide N-tetradecanoyltransferase } \\
1\end{array}$ & + & & & & & & & & & & + & [194] \\
\hline 2 & NMT2 & $\begin{array}{l}\text { Glycylpeptide N-tetradecanoyltransferase } \\
2\end{array}$ & + & & & & & & & $\mathrm{d}$ & & & + & \\
\hline 2 & NOC2L & Nucleolar complex protein 2 homolog & & & & + & & & & $d$ & & + & & \\
\hline 7 & NOLC1 & $\begin{array}{l}\text { Nucleolar phosphoprotein p130 (nucleolar } \\
\text { and coiled-body phosphoprotein 1) }\end{array}$ & & & + & & & & $\mathrm{u}$ & $d$ & & & + & \\
\hline 9 & NOP2 & $\begin{array}{l}\text { Probable } 28 S \text { rRNA (cytosine }(4447)-C(5)- \\
\text { methyltransferase }\end{array}$ & & & & + & & & $\mathrm{u}$ & & & + & & \\
\hline 15 & NPEPPS & $\begin{array}{l}\text { Puromycin-sensitive aminopeptidase, } \\
\text { metalloproteinase MP100 }\end{array}$ & + & + & & + & & & & & & & + & \\
\hline 7 & NPM1 & $\begin{array}{l}\text { Nucleophosmin (nucleolar phosphoprotein, } \\
\text { numatrin) }\end{array}$ & + & + & + & + & + & + & $\mathrm{u}$ & d & Orf9c & + & + & [195] \\
\hline 2 & NRCAM & Neuronal cell adhesion molecule & & & & + & & & $\mathrm{u}$ & $d$ & & & + & [196] \\
\hline 3 & NSFL1C & NSFL1 cofactor p47 & & + & & & & & $\mathrm{u}$ & & & & + & \\
\hline 8 & NUDC & $\begin{array}{l}\text { Nuclear distribution C, Dynein complex } \\
\text { regulator }\end{array}$ & + & + & + & & & & & $d$ & Nsp12 & & + & \\
\hline 4 & NUDT21 & $\begin{array}{l}\text { Cleavage and polyadenylation specificity } \\
\text { factor } 5\end{array}$ & + & + & & + & & & & $d$ & & & + & \\
\hline
\end{tabular}


bioRxiv preprint doi: https://doi.org/10.1101/2021.07.30.454526; this version posted August 4, 2021. The copyright holder for this preprint (which was not certified by peer review) is the author/funder, who has granted bioRxiv a license to display the preprint in perpetuity. It is made available under aCC-BY 4.0 International license.

\begin{tabular}{|c|c|c|c|c|c|c|c|c|c|c|c|c|c|c|}
\hline 2 & NUDT3 & $\begin{array}{l}\text { Diphosphoinositol polyphosphate } \\
\text { phosphohydrolase }\end{array}$ & & + & & & & & & & & & + & \\
\hline 4 & NUDT5 & Nudix hydrolase 5 & + & + & + & & + & & & $d$ & & & + & \\
\hline 3 & NUMA1 & Nuclear mitotic apparatus protein 1 & + & & & & & & $\mathrm{u}$ & $d$ & & & + & [197] \\
\hline 2 & OLA1 & Obg-like ATPase 1 & & & & + & & & $\mathrm{u}$ & & & & + & \\
\hline 2 & OTUB1 & Ubiquitin thioesterase protein OTUB1 & & + & & & & & & & & & + & \\
\hline 5 & $\mathrm{P} 3 \mathrm{H} 1$ & $\begin{array}{l}\text { Basement membrane chondroitin sulfate } \\
\text { proteoglycan }\end{array}$ & + & & & & & & $\mathrm{u}$ & & & & + & \\
\hline 2 & P3H3 & Prolyl 3-hydroxylase 3, LEPREL2 & + & & & & & & & $d$ & & + & & \\
\hline 2 & $\mathrm{P} 3 \mathrm{H} 4$ & $\begin{array}{l}\text { ER protein SC65, nucleolar autoantigen } \\
\text { No55 }\end{array}$ & + & & & & & & & & M & + & & [198] \\
\hline 2 & P4HA2 & Prolyl 4-hydroxylase subunit alpha-2 & + & & & & & & & $d$ & & & + & \\
\hline 18 & $\mathrm{P} 4 \mathrm{HB}$ & Protein disulfide-isomerase & + & + & + & + & + & + & $\mathrm{u}$ & d & $\begin{array}{l}\text { Nsp7 } \\
\text { Orf3b }\end{array}$ & & + & [199] \\
\hline 14 & PA2G4 & Proliferation-associated protein 2G4 & + & + & & & & & $\mathrm{u}$ & $d$ & & & + & \\
\hline 22 & PABPC1 & Poly(A)-binding protein 1 & + & + & & + & & & & $d$ & $\mathrm{~N}$ & + & & [200] \\
\hline 9 & PABPC3 & Poly $(\mathrm{A})$-binding protein 3 & & & + & & + & + & & $d$ & & + & + & \\
\hline 16 & PABPC4 & Poly(A)-binding protein 4, APP1 & + & & + & + & & + & & $d$ & $\mathrm{~N}$ & + & + & [201] \\
\hline 4 & PAF1 & $\begin{array}{l}\text { RNA polymerase II-associated factor } 1 \\
\text { homolog }\end{array}$ & & & & + & & & & $d$ & & + & & \\
\hline 2 & PAFAH1B2 & $\begin{array}{l}\text { Platelet-activating factor acetylhydrolase } \\
\text { IB subunit beta }\end{array}$ & & + & & + & & & $\mathrm{u}$ & $d$ & & & + & \\
\hline 3 & PAFAH1B3 & $\begin{array}{l}\text { Platelet-activating factor acetylhydrolase } \\
\text { IB subunit gamma }\end{array}$ & & + & & + & & & u & & $\begin{array}{c}\text { Nsp12 } \\
\text { Nsp5 } \\
\text { Orf3b }\end{array}$ & & + & \\
\hline 6 & PAICS & Multifunctional protein ADE2 & & + & & & & & & d & & & + & \\
\hline 2 & PARP1 & Poly [ADP-ribose] polymerase 1 & & + & & & & & $\mathrm{u}$ & $d$ & & & + & \\
\hline 3 & PARVA & Alpha-parvin & + & & & & & & $\mathrm{u}$ & & & & + & \\
\hline 8 & PCNA & Proliferating cell nuclear antigen & + & + & + & + & + & + & $\mathrm{u}$ & $d$ & & & + & [202] \\
\hline 2 & PDCD10 & Programmed cell death protein 10 & & + & & & & & & & & & + & \\
\hline 21 & PDIA3 & Protein disulfide-isomerase A3 & + & + & & + & & & $\mathrm{u}$ & $d$ & $\begin{array}{c}M \\
\text { Orf3a } \\
\text { Orf3b } \\
\text { Orf8 } \\
\text { Orf9c }\end{array}$ & & + & [203] \\
\hline 34 & PDIA4 & Protein disulfide-isomerase A4 & + & + & + & + & + & + & $\mathrm{u}$ & d & $\begin{array}{c}\text { Nsp16 } \\
\text { Nsp7 } \\
\text { Orf3b }\end{array}$ & & + & [204] \\
\hline 10 & PDIA6 & Protein disulfide-isomerase A6 & + & + & + & + & + & + & $\mathrm{u}$ & d & & & + & [205] \\
\hline 6 & PELP1 & $\begin{array}{l}\text { Proline-, glutamic acid-, leucine-rich } \\
\text { protein } 1\end{array}$ & & & & + & & & & d & & + & & \\
\hline 2 & PES1 & Pescadillo homolog & & & & + & & & & $d$ & & + & & \\
\hline 7 & PFAS & $\begin{array}{l}\text { Formylglycinamide ribonucleotide } \\
\text { amidotransferase }\end{array}$ & & + & & + & & & & & $\begin{array}{c}\text { Nsp7 } \\
\text { Nsp12 } \\
\text { Nsp15 } \\
\text { Nsp16 }\end{array}$ & & + & \\
\hline 3 & PFDN2 & Prefoldin subunit 2 & + & + & & & & & u & & $\begin{array}{l}\text { Nsp12 } \\
\text { Nsp15 } \\
\text { Orf10 }\end{array}$ & & + & [206] \\
\hline 4 & PFDN3 & $\begin{array}{l}\text { Prefoldin subunit } 3 \text {, von hippel-lindau- } \\
\text { binding protein } 1 \text {, VBP1 }\end{array}$ & + & + & + & & + & & & d & $\begin{array}{l}\text { Nsp12 } \\
\text { Nsp15 }\end{array}$ & & + & \\
\hline 2 & PFKP & $\begin{array}{l}\text { ATP-dependent 6-phofructokinase, platelet } \\
\text { type }\end{array}$ & & & & + & & & $\mathrm{u}$ & $d$ & Orf7a & & + & [207] \\
\hline 9 & PFN1 & Profilin-1 & + & + & + & & & & $\mathrm{u}$ & d & & & + & [208] \\
\hline 2 & PFN2 & Profilin-2 & + & & & & & & u & & & & + & [181] \\
\hline 4 & PGAM1 & Phosphoglycerate mutase 1 & & + & & & & & $\mathrm{u}$ & d & & & + & [209] \\
\hline 4 & PGAM2 & Phosphoglycerate mutase 2 & & + & & + & & & & & & & + & \\
\hline 9 & PGD & $\begin{array}{l}\text { 6-phosphogluconate dehydrogenase, } \\
\text { decarboxylating }\end{array}$ & & & & + & & & $\mathrm{u}$ & $d$ & & & + & \\
\hline 3 & PGLS & 6-phosphogluconolactonase & & + & & & & & $\mathrm{u}$ & & & & + & \\
\hline 3 & PHGDH & D-3-phosphoglycerate dehydrogenase & & + & & & & & $\mathrm{u}$ & d & & & + & [210] \\
\hline 2 & PLA2G4A & Cytosolic phospholipase a2 & & & + & & & & & & & & + & \\
\hline 10 & PLCG2 & $\begin{array}{l}\text { 1-phosphatidylinositol-4,5-bisphosphate } \\
\text { phosphodiesterase gamma-2 }\end{array}$ & & + & & & & & $\mathrm{u}$ & & & & + & \\
\hline 2 & PLD3 & $\begin{array}{l}\text { Phospholipase D3, 5'-3' exonuclease } \\
\text { PLD3 }\end{array}$ & & & & + & & & u & $d$ & $\begin{array}{l}\text { Nsp2 } \\
\text { Nsp3 }\end{array}$ & & + & \\
\hline
\end{tabular}


bioRxiv preprint doi: https://doi.org/10.1101/2021.07.30.454526; this version posted August 4, 2021. The copyright holder for this preprint (which was not certified by peer review) is the author/funder, who has granted bioRxiv a license to display the preprint in perpetuity. It is made available under aCC-BY 4.0 International license.

\begin{tabular}{|c|c|c|c|c|c|c|c|c|c|c|c|c|c|c|}
\hline & & & & & & & & & & & $\begin{array}{c}\text { Nsp5 } \\
\text { Orf6 } \\
\text { Orf7b } \\
\text { Orf8 } \\
\text { Orf10 }\end{array}$ & & & \\
\hline 91 & PLEC & Plectin-1, PLEC1 & + & & & + & & & $\mathrm{u}$ & d & & + & + & [211] \\
\hline 5 & PLOD1 & $\begin{array}{l}\text { Procollagen-lysine, 2-oxoglutarate 5- } \\
\text { dioxygenase } 1\end{array}$ & + & & & & & & & $\mathrm{d}$ & & & + & \\
\hline 5 & PLOD3 & $\begin{array}{l}\text { Multifunctional procollagen lysine } \\
\text { hydroxylase and glycosyltransferase LH3 }\end{array}$ & + & & & & & & & & & & + & \\
\hline 2 & PLS1 & Plastin-1 & & & & + & & & & $d$ & & & + & \\
\hline 30 & PLS2 & Plastin-2, LCP1 & + & + & + & + & + & & $\mathrm{u}$ & $d$ & & & + & [212] \\
\hline 6 & PLS3 & Plastin-3 & + & & & + & & & $\mathrm{u}$ & $d$ & & & + & \\
\hline 2 & PMPCB & $\begin{array}{l}\text { Mitochondrial-processing peptidase } \\
\text { subunit beta }\end{array}$ & & + & & & & & & $d$ & M & & + & \\
\hline 2 & POP1 & $\begin{array}{l}\text { Ribonucleases P/MRP protein subunit } \\
\text { POP1 }\end{array}$ & & & & + & & & $\mathrm{u}$ & & & + & & [213] \\
\hline 3 & POR & NADPH--cytochrome P450 reductase & & & & + & & & $\mathrm{u}$ & $d$ & $\begin{array}{l}\text { Nsp2 } \\
\text { Orf6 }\end{array}$ & + & & \\
\hline 8 & PPA1 & Inorganic pyrophosphatase & & + & & + & & & $\mathrm{u}$ & & Orf3 & & + & [214] \\
\hline 3 & PPAT & Amidophosphoribosyltransferase & & + & & & & & & $d$ & & & + & \\
\hline 10 & PPIB & Peptidyl-prolyl cis-trans isomerase & + & + & & + & & & $\mathrm{u}$ & $d$ & Orf8 & & + & [215] \\
\hline 3 & PPM1G & Protein phosphatase 1G (PPM1C) & & & + & & & + & & & Orf9b & & + & \\
\hline 2 & PPP1R7 & $\begin{array}{l}\text { Protein phosphatase } 1 \text { regulatory subunit } \\
7 \text { (subunit 22) }\end{array}$ & & + & & & + & & $\mathrm{u}$ & & & & + & \\
\hline 7 & PPP2R1A & $\begin{array}{l}\text { Serine/threonine-protein phosphatase } 2 \mathrm{~A} \\
65 \mathrm{kDa} \text { regulatory subunit } \mathrm{A} \text { alpha isoform }\end{array}$ & & + & + & & + & & & $\mathrm{d}$ & & & + & \\
\hline 6 & PRDX1 & Peroxiredoxin-1 & & + & & + & & & $\mathrm{u}$ & $d$ & & & + & [216] \\
\hline 5 & PRDX3 & $\begin{array}{l}\text { Thioredoxin-dependent peroxide } \\
\text { reductase }\end{array}$ & + & + & & + & & & $\mathrm{u}$ & $\mathrm{d}$ & & & + & [217] \\
\hline 3 & PRDX4 & Peroxiredoxin-4 & + & & & + & & & $\mathrm{u}$ & $d$ & Orf3a & & + & [218] \\
\hline 2 & PRKAR2A & $\begin{array}{l}\text { Protein kinase CAMP-dependent type II } \\
\text { regulatory alpha }\end{array}$ & + & & & & & & $\mathrm{u}$ & & $\begin{array}{l}\text { Nsp1 } \\
\text { Orf9b }\end{array}$ & & + & {$[48]$} \\
\hline 2 & PRKCDBP & Protein kinase $\mathrm{C}$ delta-binding protein & + & & & & & & & & & & + & \\
\hline 11 & PRKCSH & Protein kinase $\mathrm{C}$ substrate $80 \mathrm{~K}-\mathrm{H}$ & + & + & + & & + & + & & $d$ & $\begin{array}{c}\text { Nsp6 } \\
\text { Orf3 } \\
\text { Orf3a } \\
\text { S }\end{array}$ & & + & \\
\hline 17 & PRKDC & $\begin{array}{l}\text { DNA-dependent protein kinase catalytic } \\
\text { subunit (DNA-PKcs) }\end{array}$ & + & & + & + & & + & $\mathrm{u}$ & $d$ & $\begin{array}{c}\text { M } \\
\text { Nsp4 }\end{array}$ & + & + & [219] \\
\hline 5 & PRMT1 & $\begin{array}{l}\text { Protein arginine N-methyltransferase } \\
1 \text { (Histone-arginine N-methyltransferase) }\end{array}$ & + & + & + & + & + & + & & $\mathrm{d}$ & & & + & [220] \\
\hline 24 & PRPF8 & $\begin{array}{l}\text { Pre-mRNA-processing-splicing factor } 8 \\
\text { (U5 snRNP-specific protein } 220 \mathrm{kDa} \text { ) }\end{array}$ & + & & & + & & + & $\mathrm{u}$ & $d$ & & + & + & {$[57]$} \\
\hline 2 & PRPSAP2 & $\begin{array}{l}\text { Phosphoribosyl pyrophosphate } \\
\text { synthetase-associated protein } 2\end{array}$ & & + & & & & & $\mathrm{u}$ & & & & + & \\
\hline 2 & PSAP & Proactivator polypeptide, Prosaposin & + & & & & & & $\mathrm{u}$ & $d$ & & & + & \\
\hline 6 & PSAT1 & Phosphoserine aminotransferase 1 & & & & + & & & $\mathrm{u}$ & $\mathrm{d}$ & $\begin{array}{l}\text { Orf3 } \\
\text { Orf7a }\end{array}$ & & + & \\
\hline 3 & PSMA1 & Proteasome subunit alpha type-1 & & + & + & & + & & & $d$ & Orf3b & & + & {$[25]$} \\
\hline 2 & PSMA2 & Proteasome subunit alpha type-2 & & + & & & + & + & & d & & & + & \\
\hline 6 & PSMA3 & Proteasome subunit alpha type-3, C8 & + & + & & & + & & $\mathrm{u}$ & $\mathrm{d}$ & $\begin{array}{c}\text { Nsp2 } \\
\text { Nsp4 } \\
\text { Nsp7 } \\
\text { Nsp10 } \\
\text { Nsp12 }\end{array}$ & & + & [221] \\
\hline 5 & PSMA4 & Proteasome subunit alpha type- $4, \mathrm{C} 9$ & + & + & & & & & $\mathrm{u}$ & & & & + & [25] \\
\hline 5 & PSMA5 & Proteasome subunit alpha type- 5 & + & + & + & & + & + & $\mathrm{u}$ & & Orf3b & & + & [222] \\
\hline 8 & PSMA6 & Proteasome subunit alpha type- 6 & + & + & & & & & $\mathrm{u}$ & $d$ & Orf3b & & + & \\
\hline 6 & PSMA7 & Proteasome subunit alpha type-7 & + & + & + & & + & + & $\mathrm{u}$ & $d$ & & & + & [223] \\
\hline 3 & PSMA8 & Proteasome subunit alpha type 7-like & & & + & & + & & & & & & + & [223] \\
\hline 5 & PSMB1 & Proteasome subunit beta type- 1 & + & + & & & & & & & & & + & [224] \\
\hline 3 & PSMB3 & Proteasome subunit beta type-3 & + & + & & & + & & & d & Orf3b & & + & [221] \\
\hline 7 & PSMB4 & Proteasome subunit beta type- 4 & + & + & + & & + & & & & Orf3b & & + & {$[25]$} \\
\hline 3 & PSMB6 & Proteasome subunit beta type- 6 & + & + & + & & & & & $d$ & Orf3b & & + & \\
\hline 5 & PSMB7 & Proteasome subunit beta type- 7 & + & + & & & + & & & $d$ & & & + & [221] \\
\hline 3 & PSMB8 & Proteasome subunit beta type- 8 & & + & & & & & $\mathrm{u}$ & d & & & + & \\
\hline
\end{tabular}


bioRxiv preprint doi: https://doi.org/10.1101/2021.07.30.454526; this version posted Auqust 4, 2021. The copyright holder for this preprint (which was not certified by peer review) is the author/funder, who has granted bioRxiv a license to display the preprint in perpetuity. It is made available under aCC-BY 4.0 International license.

\begin{tabular}{|c|c|c|c|c|c|c|c|c|c|c|c|c|c|c|}
\hline 4 & PSMB9 & Proteasome subunit beta type- 9 & & + & & & & & u & d & & & + & \\
\hline 2 & PSMC1 & 26 s Proteasome regulatory subunit 4 & & & & & + & & & $d$ & Orf6 & & + & \\
\hline 2 & PSMC3 & $26 \mathrm{~S}$ protease regulatory subunit $6 \mathrm{~A}$ & & + & & & & & & d & Orf6 & & + & \\
\hline 5 & PSMD1 & $\begin{array}{l}26 \mathrm{~S} \text { proteasome non-ATPase regulatory } \\
\text { subunit } 1\end{array}$ & + & + & & + & & & $\mathrm{u}$ & & $\begin{array}{l}\text { Nsp7 } \\
\text { Orf6 } \\
\text { Orf8 }\end{array}$ & + & + & \\
\hline 9 & PSMD11 & $\begin{array}{l}\text { Proteasome } 26 \mathrm{~S} \text { non-ATPase regulatory } \\
\text { subunit } 11\end{array}$ & & + & + & & & & $\mathrm{u}$ & & & & + & \\
\hline 3 & PSMD12 & $\begin{array}{l}\text { 26S proteasome non-ATPase regulatory } \\
\text { subunit } 12\end{array}$ & + & + & & & & & & $d$ & & + & + & \\
\hline 3 & PSMD13 & Proteasome $26 \mathrm{~S}$ non-ATPase subunit 13 & + & & & & & + & & $d$ & & & + & [225] \\
\hline 2 & PSMD14 & $\begin{array}{l}\text { 26S proteasome non-ATPase regulatory } \\
\text { subunit } 14\end{array}$ & & + & & & & & & & & & + & \\
\hline 8 & PSMD3 & $\begin{array}{l}\text { 26S proteasome non-ATPase regulatory } \\
\text { subunit } 3\end{array}$ & & + & & & & & & d & & & + & \\
\hline 9 & PSMD6 & $\begin{array}{l}\text { 26S proteasome non-ATPase regulatory } \\
\text { subunit } 6\end{array}$ & + & + & & + & & + & & & & & + & \\
\hline 2 & PSMD7 & $\begin{array}{l}\text { 26S proteasome non-ATPase regulatory } \\
\text { subunit } 7\end{array}$ & + & & & & & & $\mathrm{u}$ & & & & + & \\
\hline 11 & PSME1 & Proteasome activator complex subunit 1 & & + & & & & & u & & Nsp15 & & + & \\
\hline 8 & PSME2 & Proteasome activator complex subunit 2 & & + & & & & & $\mathrm{u}$ & & & & + & \\
\hline 4 & PSME3 & Proteasome activator complex subunit 3 & & + & + & & + & & & $d$ & Nsp16 & & + & [226] \\
\hline 2 & PSPH & Phosphoserine phosphatase & & + & & & & & & & & & + & \\
\hline 6 & PTBP1 & $\begin{array}{l}\text { Polypyrimidine tract-binding protein, } \\
\text { hnRNP I }\end{array}$ & + & + & & & & & u & $d$ & & & + & [227] \\
\hline 2 & PTBP3 & Polypyrimidine tract-binding protein, ROD1 & + & + & & & & & $\mathrm{u}$ & d & & & + & [227] \\
\hline 16 & PTCD3 & $\begin{array}{l}\text { Pentatricopeptide repeat-containing } \\
\text { protein } 3 \text {, mitochondrial, MRPS } 39\end{array}$ & + & & & + & & & & & & + & & \\
\hline 2 & PTGES3 & $\begin{array}{l}\text { Prostaglandin E synthase } 3 \text { (telomerase- } \\
\text { binding protein p23) (hsp90 co- } \\
\text { chaperone) (progesterone rec) }\end{array}$ & & + & + & & + & & & $d$ & & & + & \\
\hline 4 & PTMA & Prothymosin alpha & & & + & & + & + & $\mathrm{u}$ & $d$ & & & + & [228] \\
\hline 2 & PTPN6 & $\begin{array}{l}\text { Tyrosine-protein phosphatase non- } \\
\text { receptor type } 6\end{array}$ & & + & & & & & u & $d$ & & & + & \\
\hline 2 & PUF60 & Poly(U)-binding-splicing factor PUF60 & + & & & & & & u & & & & + & [229] \\
\hline 18 & PUM1 & Pumilio homolog 1 & & & & + & & & & d & & + & & \\
\hline 3 & PURA & Transcriptional activator protein Pur-alpha & & & & + & & & $\mathrm{u}$ & d & & + & & \\
\hline 2 & PUS1 & tRNA pseudouridine synthase $A$ & & + & & & & & & & & & + & \\
\hline 2 & PZP & $\begin{array}{l}\text { Pregnancy zone protein, alpha-2- } \\
\text { macroglobulin like }\end{array}$ & + & & & & & & & $d$ & & & + & [230] \\
\hline 4 & QARS & $\begin{array}{l}\text { Bifunctional glutamate/proline-tRNA ligase, } \\
\text { EPRS, EPRS1 }\end{array}$ & + & + & & + & & & $\mathrm{u}$ & & & + & + & [100] \\
\hline 3 & RAB1A & Ras-related protein Rab-1A & + & & & + & & & & d & $\begin{array}{l}\text { Nsp7 } \\
\text { Orf3 } \\
\text { Orf7b }\end{array}$ & + & & \\
\hline 5 & RAB7A & Ras-related protein Rab-7a & + & + & & & & & $\mathrm{u}$ & $d$ & $\begin{array}{l}\text { Nsp7 } \\
\text { Orf3 } \\
\text { Orf7b }\end{array}$ & & + & \\
\hline 3 & RAD23A & $\begin{array}{l}\text { UV excision repair protein RAD23 } \\
\text { homolog } A\end{array}$ & + & + & & & & & & $d$ & & & + & [231] \\
\hline 5 & RAD23B & $\begin{array}{l}\text { UV excision repair protein RAD23 } \\
\text { homolog B }\end{array}$ & + & & & & & & $\mathrm{u}$ & $d$ & $\begin{array}{c}\text { Orf3a } \\
\text { Orf3b } \\
\text { Orf7a } \\
\text { Orf9c }\end{array}$ & & + & [231] \\
\hline 6 & RALY & RNA binding protein, autoantigen p542 & + & & & + & & & $\mathrm{u}$ & $d$ & Orf9c & + & & [232] \\
\hline 3 & RANBP1 & Ran-specific GTPase-activating protein & & + & & & & & $\mathrm{u}$ & d & & & + & \\
\hline 2 & RANBP6 & Ran-binding protein 6 & & + & & & & & & $d$ & Orf7a & & + & \\
\hline 2 & RANGAP1 & Ran GTPase-activating protein 1 & & & + & + & & & & $d$ & & + & & [165] \\
\hline 3 & RARS & $\begin{array}{l}\text { Arginyl-tRNA synthetase, cytoplasmic, } \\
\text { RARS1 }\end{array}$ & & & & + & & & $\mathrm{u}$ & & & + & & [39] \\
\hline 5 & RBBP4 & Chromosome assembly factor 1 subunit $\mathrm{C}$ & + & & & + & & & & $d$ & & + & & [233] \\
\hline 3 & RBBP7 & Histone-binding protein rbbp7 & & & + & & + & & $\mathrm{u}$ & d & & & + & \\
\hline 2 & RBM3 & Putative RNA-binding protein 3 & + & & & & & & $u$ & d & Orf8 & & + & \\
\hline 3 & RBM8A & RNA-binding protein $8 \mathrm{~A}$ & & & & + & & & $u$ & & & + & & \\
\hline 2 & RBMXL2 & RNA-binding motif protein X-linked-like-2 & + & & & & & & & & & & + & \\
\hline 2 & RCN3 & Reticulocalbin-3 & + & & & & & & & & & & + & \\
\hline 8 & RDX & Radixin & + & + & + & + & & & $\mathrm{u}$ & $d$ & Nsp13 & & + & [234] \\
\hline
\end{tabular}


bioRxiv preprint doi: https://doi.org/10.1101/2021.07.30.454526; this version posted Auqust 4, 2021. The copyright holder for this preprint (which was not certified by peer review) is the author/funder, who has granted bioRxiv a license to display the preprint in perpetuity. It is made available under aCC-BY 4.0 International license.

\begin{tabular}{|c|c|c|c|c|c|c|c|c|c|c|c|c|c|c|}
\hline 2 & RMI2 & $\begin{array}{l}\text { RecQ-mediated genome instability protein } \\
2\end{array}$ & & & + & & & & & & & + & & \\
\hline 3 & RNPEP & Arginine aminopeptidase, APB & & & & + & & & & & Orf3 & & + & \\
\hline 2 & RNPS1 & $\begin{array}{l}\text { RNA-binding protein with serine-rich } \\
\text { domain } 1\end{array}$ & & & & + & & & u & $\mathrm{d}$ & & + & & \\
\hline 4 & R052 & $\begin{array}{l}\text { E3 ubiquitin-protein ligase TRIM21 } \\
\text { (Ro/SS-A), TRIM21 }\end{array}$ & & & & & & + & $\mathrm{u}$ & $d$ & & + & & \\
\hline 4 & $\mathrm{RO} 60$ & $60 \mathrm{kDa}$ SS-A/Ro ribonucleoprotein & + & & & + & & & $\mathrm{u}$ & & & + & & [235] \\
\hline 2 & RPA3 & Replication protein A 14 kda subunit & & & & & + & & & & & & + & [236] \\
\hline 3 & RPF2 & $\begin{array}{l}\text { Ribosome production factor } 2 \text { homolog, } \\
\text { BXDC1 }\end{array}$ & + & & & + & & & & & & + & & \\
\hline 2 & RPL10A & $60 \mathrm{~S}$ ribosomal protein $\mathrm{L} 10 \mathrm{a}$ & & + & & + & & & & & & + & & \\
\hline 2 & RPL11 & $60 S$ ribosomal protein L11 & + & + & & + & & & u & & & + & & \\
\hline 4 & RPL12 & $60 S$ ribosomal protein L12 & + & & + & + & & & u & d & & + & & [237] \\
\hline 2 & RPL15 & $60 S$ ribosomal protein L15 & + & + & + & + & & & & $d$ & & + & & \\
\hline 3 & RPL18 & $60 S$ ribosomal protein L18 & + & & + & + & & & & $d$ & & + & & \\
\hline 2 & RPL22 & $60 S$ ribosomal protein L22 & + & + & + & & + & & & $d$ & & & + & [93] \\
\hline 2 & RPL23A & Ribosomal protein L23a & & & + & & & & $\mathrm{u}$ & & & & + & \\
\hline 2 & RPL26L1 & $\begin{array}{l}\text { 60S ribosomal protein L26-like 1, } \\
\text { RPL26P1 }\end{array}$ & & & + & + & & & & & Orf9b & + & & \\
\hline 3 & RPL3 & $\begin{array}{l}\text { 60s ribosomal protein L3 (hiv-1 tar rna- } \\
\text { binding protein b) }\end{array}$ & & & + & & & & $\mathrm{u}$ & $d$ & & + & & \\
\hline 2 & RPL31 & $60 S$ ribosomal protein L31 & & & + & & & & $\mathrm{u}$ & $d$ & & & + & \\
\hline 2 & RPL35A & 60S ribosomal protein L35a & & & & + & & & $\mathrm{u}$ & $d$ & & + & & [238] \\
\hline 2 & RPL4 & $60 S$ ribosomal protein L4 & & & & + & & & $\mathrm{u}$ & $d$ & & + & & \\
\hline 17 & RPL5 & $60 S$ ribosomal protein L5 & + & & + & + & + & + & & d & & + & + & [239] \\
\hline 11 & RPL6 & $60 S$ ribosomal protein L6 & + & & + & + & + & + & $\mathrm{u}$ & $d$ & & + & & [181] \\
\hline 9 & RPL7 & 60S ribosomal protein L7, RPL7P32 & + & & + & + & + & + & $\mathrm{u}$ & $d$ & & + & & [93] \\
\hline 4 & RPL7A & $60 S$ ribosomal protein L7A & & & + & + & & & $\mathrm{u}$ & d & & + & & [238] \\
\hline 2 & RPL8 & $60 S$ ribosomal protein L8 & & & + & + & & & $\mathrm{u}$ & $d$ & & + & & {$[165]$} \\
\hline 8 & RPLP0 & $60 \mathrm{~S}$ acidic ribosomal protein $\mathrm{P0}$ & + & + & + & + & + & & $\mathrm{u}$ & $\mathrm{d}$ & & + & & [240] \\
\hline 2 & RPLP1 & $60 S$ acidic ribosomal protein $\mathrm{P} 1$ & & & & + & & & u & d & & + & & [241] \\
\hline 4 & RPLP2 & $60 S$ acidic ribosomal protein P2 & + & & + & + & + & & $\mathrm{u}$ & $\mathrm{d}$ & & + & + & [241] \\
\hline 2 & RPS15A & 40s ribosomal protein $\mathrm{S} 15 \mathrm{a}$ & & + & + & + & & & $\mathrm{u}$ & & & + & & \\
\hline 3 & RPS18 & 40S ribosomal protein S18 & + & & & + & & & $\mathrm{u}$ & $d$ & $\begin{array}{c}\text { Nsp13 } \\
\text { Orf8 } \\
\text { Orf10 }\end{array}$ & + & & [165] \\
\hline 3 & RPS19 & 40S ribosomal protein S19 & + & & & & & & & $d$ & Orf9c & & + & [238] \\
\hline 3 & RPS2 & $40 S$ ribosomal protein S2 & + & & + & + & & & u & $\mathrm{d}$ & & + & & [39] \\
\hline 2 & RPS25 & $40 S$ ribosomal protein $\$ 25$ & & + & + & & & & u & d & Orf8 & + & + & [93] \\
\hline 3 & RPS27A & $\begin{array}{l}\text { Ubiquitin-40S ribosomal protein S27a, } \\
\text { UBA80, UBCEP1 }\end{array}$ & & + & & + & & & $\mathrm{u}$ & $d$ & $\begin{array}{c}\text { Nsp4 } \\
\mathrm{S}\end{array}$ & + & & [93] \\
\hline 6 & RPS3 & 40 S ribosomal protein S3 & + & + & + & + & & & u & $d$ & Orf8 & + & + & [242] \\
\hline 3 & RPS3A & 40S ribosomal protein S3a & + & + & + & & + & & $\mathrm{u}$ & $d$ & Orf8 & + & + & \\
\hline 3 & RPS4X & 40S ribosomal protein $\mathrm{S} 4, \mathrm{X}$ isoform & + & & & + & & & & $d$ & Orf8 & + & & {$[25]$} \\
\hline 3 & RPS6 & 40 S ribosomal protein S6 & & & + & + & & & $\mathrm{u}$ & $d$ & Nsp6 & + & & [238] \\
\hline 3 & RPS7 & $40 S$ ribosomal protein S7 & & + & + & & + & & $\mathrm{u}$ & $d$ & & & + & [93] \\
\hline 2 & RPS8 & $40 \mathrm{~S}$ ribosomal protein S8 & + & & & + & & & $\mathrm{u}$ & $d$ & & + & & \\
\hline 8 & RPS9 & $40 S$ ribosomal protein S9 & + & & + & + & & + & & $d$ & & + & & [238] \\
\hline 5 & RPSA & 40 S ribosomal protein SA & & + & & + & & & $\mathrm{u}$ & $d$ & & & + & [243] \\
\hline 13 & RRBP1 & Ribosome-binding protein 1 & + & & & + & & & $\mathrm{u}$ & $d$ & Orf8 & + & + & \\
\hline 11 & RRP12 & RRP12-like protein & & & & + & & & $\mathrm{u}$ & & & + & & \\
\hline 4 & RRP9 & $\begin{array}{l}\text { U3 small nucleolar RNA-interacting protein } \\
2\end{array}$ & & & + & + & & & $\mathrm{u}$ & $d$ & $\mathrm{~N}$ & + & & [244] \\
\hline 4 & RRS1 & $\begin{array}{l}\text { Ribosome biogenesis regulatory protein } \\
\text { homolog }\end{array}$ & & & & + & & & u & & & + & & \\
\hline 5 & RSL1D1 & Ribosomal L1 domain-containing protein 1 & & & & + & & & $\mathrm{u}$ & $d$ & & + & & \\
\hline 6 & RUVBL1 & $\begin{array}{l}\text { RuvB-like } 1 \text {, tata box-binding protein- } \\
\text { interacting protein }\end{array}$ & & + & + & + & & & & & $\begin{array}{c}\text { Nsp1 } \\
\text { Nsp7 } \\
\text { Nsp12 } \\
\text { Orf9b }\end{array}$ & + & + & [245] \\
\hline 5 & RUVBL2 & RuvB-like 2 & & + & & & & & & $d$ & $\begin{array}{c}\text { Nsp1 } \\
\text { Nsp7 } \\
\text { Nsp12 } \\
\text { Orf9b }\end{array}$ & & + & [246] \\
\hline 2 & SARS & Serine-tRNA ligase, cytoplasmic, SARS1 & & + & & & & & u & & Nsp15 & & + & \\
\hline
\end{tabular}


bioRxiv preprint doi: https://doi.org/10.1101/2021.07.30.454526; this version posted Auqust 4, 2021. The copyright holder for this preprint (which was not certified by peer review) is the author/funder, who has granted bioRxiv a license to display the preprint in perpetuity. It is made available under aCC-BY 4.0 International license.

\begin{tabular}{|c|c|c|c|c|c|c|c|c|c|c|c|c|c|c|}
\hline 4 & SEPHS1 & Selenide, water dikinase & + & & & & & & & $d$ & & & + & [247] \\
\hline 2 & SEPT11 & Septin-11 & & + & & & & & & $d$ & & & + & [25] \\
\hline 2 & SEPT2 & Septin-2, NEDD5, DIFF6 & + & + & & & & & $\mathrm{u}$ & d & & & + & [248] \\
\hline 3 & SEPT7 & Septin-7 & & + & & & & & & d & & & + & [249] \\
\hline 5 & SERPINB1 & Leukocyte elastase inhibitor & & & & + & & & u & & & & + & \\
\hline 4 & SERPINB6 & Serpin B6, peptidase inhibitor 6 & & & & + & & & & & & & + & \\
\hline 8 & SERPINB9 & Serpin B9 & & + & & & & & u & d & & & + & \\
\hline 2 & SERPINC1 & Antithrombin-III & & + & & + & & & $\mathrm{u}$ & & & & + & \\
\hline 3 & SERPINE1 & Plasminogen activator inhibitor 1 & + & & & & & & $\mathrm{u}$ & $d$ & Orf8 & & + & [250] \\
\hline 4 & SERPINH1 & Serpin H1, HSP47 & + & & & & & & & $d$ & & & + & [251] \\
\hline 6 & SET & $\begin{array}{l}\text { SET nuclear proto-oncogene (Inhibitor of } \\
\text { granzyme A-activated DNase, HLA-DR- } \\
\text { associated protein II) }\end{array}$ & + & + & + & + & + & + & $\mathrm{u}$ & d & & + & + & [252] \\
\hline 2 & SF3A1 & $\begin{array}{l}\text { Splicing factor } 3 \text { subunit } 1 \text { (spliceosome- } \\
\text { associated protein 114) (sap 114) } \\
\text { (sf3a120) }\end{array}$ & & & + & & & & $\mathrm{u}$ & & & & + & \\
\hline 14 & SF3B1 & Splicing factor 3B subunit 1 & + & & & + & & & $\mathrm{u}$ & $d$ & & + & & [253] \\
\hline 13 & SF3B3 & Splicing factor 3B subunit 3, SAP130 & + & + & + & + & + & + & u & & & + & + & [253] \\
\hline 8 & SFN & 14-3-3 protein sigma, Stratifin & & & & + & & + & $\mathrm{u}$ & d & & & + & [254] \\
\hline 3 & SFPQ & Splicing factor, proline- and glutamine-rich & + & + & & & & & $\mathrm{u}$ & d & & & + & [255] \\
\hline 3 & SGTA & $\begin{array}{l}\text { Small glutamine-rich tetratricopeptide } \\
\text { repeat-containing protein alpha }\end{array}$ & & + & & & & & $\mathrm{u}$ & d & M & & + & \\
\hline 3 & SH3BGRL3 & $\begin{array}{l}\text { SH3 domain-binding glutamic acid-rich-like } \\
\text { protein } 3\end{array}$ & + & & & & & & & d & & & + & \\
\hline 2 & SHMT1 & Serine hydroxymethyltransferase, cytosolic & & + & & & & & & d & & & + & \\
\hline 9 & SHMT2 & $\begin{array}{l}\text { Serine hydroxymethyltransferase, } \\
\text { mitochondrial }\end{array}$ & & + & & & & & & d & & & + & \\
\hline 2 & SKP1 & S-phase kinase-associated protein 1 & + & & & & & & $\mathrm{u}$ & d & & & + & \\
\hline 2 & SLC1A5 & $\begin{array}{l}\text { Neutral amino acid transporter B, Simian } \\
\text { type D retrovirus receptor, Baboon M7 } \\
\text { virus receptor }\end{array}$ & & & & + & & & $\mathrm{u}$ & d & $\begin{array}{l}\text { Orf3 } \\
S\end{array}$ & + & & \\
\hline 2 & SLC2A1 & HepG2 glucose transporter, GLUT1 & & & & + & & & & $d$ & Nsp8 & + & & [256] \\
\hline 17 & SLC3A2 & $\begin{array}{l}\text { 4F2 cell-surface antigen heavy chain, } \\
\text { CD98 }\end{array}$ & + & & & + & & & $\mathrm{u}$ & $d$ & $\begin{array}{l}\text { Orf3b } \\
\text { Orf9c }\end{array}$ & + & & \\
\hline 2 & SLIRP & $\begin{array}{l}\text { SRA stem-loop-interacting RNA-binding } \\
\text { protein, mitochondrial }\end{array}$ & & + & & & & & $\mathrm{u}$ & $d$ & & & + & \\
\hline 4 & SMS & Spermine synthase & + & & & & & & $\mathrm{u}$ & $d$ & Orf3 & & + & \\
\hline 9 & SND1 & $\begin{array}{l}\text { Staphylococcal nuclease domain- } \\
\text { containing protein } 1\end{array}$ & + & + & & + & & & $\mathrm{u}$ & d & & & + & \\
\hline 15 & SNRNP200 & $\begin{array}{l}\text { U5 small nuclear ribonucleoprotein } 200 \\
\text { kDa helicase }\end{array}$ & + & & & + & & & & $d$ & & + & & [257] \\
\hline 3 & SNRNP70 & U1 small nuclear ribonucleoprotein 70 kDa & & + & + & & + & & $\mathrm{u}$ & d & & & + & [258] \\
\hline 3 & SNRPA & U1 small nuclear ribonucleoprotein $A$ & + & + & & + & & & $\mathrm{u}$ & & & & + & [259] \\
\hline 8 & SNRPA1 & U2 small nuclear ribonucleoprotein A' & & + & + & & & & & & & + & + & [260] \\
\hline 3 & SNRPB & SnRNP-associated proteins B and B' & + & & + & + & & & $\mathrm{u}$ & d & & + & & [261] \\
\hline 2 & SNRPD1 & Small nuclear ribonucleoprotein Sm D1 & + & + & & + & & & $\mathrm{u}$ & & & + & + & [262] \\
\hline 4 & SNRPD2 & Small nuclear ribonucleoprotein Sm D2 & + & + & + & + & + & & & $d$ & & + & + & [263] \\
\hline 2 & SNRPD3 & Small nuclear ribonucleoprotein Sm D3 & + & + & & & + & & & d & & + & + & [262] \\
\hline 2 & SNRPE & Small nuclear ribonucleoprotein $\mathrm{E}$ & + & + & + & & & & & d & & + & + & [264] \\
\hline 2 & SNRPG & $\begin{array}{l}\text { Small nuclear ribonucleoprotein G, } \\
\text { PBSCG }\end{array}$ & & & & + & & & & & & + & & [264] \\
\hline 2 & SOD1 & Superoxide dismutase [Cu-Zn] & & & & + & & & $\mathrm{u}$ & $d$ & & & + & [265] \\
\hline 46 & SPTAN1 & $\begin{array}{l}\text { Spectrin alpha chain, brain (spectrin, non- } \\
\text { erythroid alpha chain) }\end{array}$ & + & + & + & + & & + & $\mathrm{u}$ & $d$ & & + & + & [266] \\
\hline 29 & SPTBN1 & Spectrin beta chain, brain & + & & + & + & & & u & $d$ & & + & & [267] \\
\hline 3 & SRM & Spermidine synthase & & + & & & & & & $d$ & & & + & \\
\hline 3 & SRP14 & Signal recognition particle $14 \mathrm{kDa}$ protein & & + & & & & & $\mathrm{u}$ & d & $\begin{array}{l}\text { Nsp13 } \\
\text { Orf8 }\end{array}$ & & + & \\
\hline 2 & SRP68 & Signal recognition particle $68 \mathrm{kda}$ protein & & & + & & & & & & $\begin{array}{c}\mathrm{N} \\
\text { Nsp2 }\end{array}$ & & + & \\
\hline 2 & SRP72 & Signal recognition particle $72 \mathrm{kDa}$ protein & & & & + & & & & d & Nsp8 & + & & [268] \\
\hline 2 & SRP9 & Signal recognition particle $9 \mathrm{kda}$ protein & & & + & & & & $\mathrm{u}$ & d & & + & & \\
\hline 2 & SRRT & Arsenite-resistance protein 2 & & & & & + & & & d & & & + & \\
\hline 5 & SRSF1 & Serine/argine-rich splicing factor 1 & & + & + & + & + & & $\mathrm{u}$ & d & & + & + & [269] \\
\hline 2 & SRSF11 & $\begin{array}{l}\text { Arginine/serine-rich splicing factor } 11 \text {, } \\
\text { SRSF11 }\end{array}$ & + & & & & & & $\mathrm{u}$ & $d$ & & & + & \\
\hline
\end{tabular}


bioRxiv preprint doi: https://doi.org/10.1101/2021.07.30.454526; this version posted August 4, 2021. The copyright holder for this preprint (which was not certified by peer review) is the author/funder, who has granted bioRxiv a license to display the preprint in perpetuity. It is made available under aCC-BY 4.0 International license.

\begin{tabular}{|c|c|c|c|c|c|c|c|c|c|c|c|c|c|c|}
\hline 3 & SRSF2 & $\begin{array}{l}\text { Arginine/serine-rich splicing factor } 2 \text {, } \\
\text { SFRS2 }\end{array}$ & + & + & + & & & & $\mathrm{u}$ & $d$ & & & + & [65] \\
\hline 2 & SRSF3 & $\begin{array}{l}\text { Serine/arginine-rich splicing factor } 3 \text {, } \\
\text { SFRS3 }\end{array}$ & & & & & + & & & & & & + & [270] \\
\hline 4 & SRSF4 & $\begin{array}{l}\text { Splicing factor, arginine/serine-rich } 4 \\
\text { (srp75) }\end{array}$ & & & + & & & & & & & & + & \\
\hline 2 & SRSF5 & $\begin{array}{l}\text { Serine/arginine-rich splicing factor } 5 \text {, } \\
\text { SRP40 }\end{array}$ & & & + & & + & & $\mathrm{u}$ & $d$ & & & + & [271] \\
\hline 2 & SRSF6 & Splicing factor, arginine/serine-rich 6 & & & + & & & & $\mathrm{u}$ & $d$ & & & + & \\
\hline 3 & SRSF7 & $\begin{array}{l}\text { Serine /arginine-rich splicing factor } 7 \text {, } \\
\text { SRSF7 }\end{array}$ & + & & + & & + & + & $\mathrm{u}$ & & & + & + & [271] \\
\hline 2 & SRSF8 & Serine/arginine-rich splicing factor 8 & & & + & & + & & & $d$ & & & + & \\
\hline 11 & SSB & $\begin{array}{l}\text { Lupus la protein (sjoegren syndrome type } \\
\text { b antigen) (La/SSB) }\end{array}$ & + & + & + & + & + & + & $\mathrm{u}$ & d & & + & + & [41] \\
\hline 9 & SSBP1 & $\begin{array}{l}\text { Single-stranded DNA-binding protein, } \\
\text { mitochondrial }\end{array}$ & + & + & & + & & & & & $\mathrm{N}$ & + & & \\
\hline 8 & SSRP1 & $\begin{array}{l}\text { Fact complex subunit ssrp1 (facilitates } \\
\text { chromatin transcription complex subunit } \\
\text { ssrp1) (factp80) (chromatin- specific } \\
\text { transcription elongation factor } 80 \mathrm{kda} \text { ) }\end{array}$ & + & & + & + & & & $\mathrm{u}$ & $d$ & & + & & [272] \\
\hline 6 & ST13 & $\begin{array}{l}\text { Hsc70-interacting protein (hip) } \\
\text { (suppression of tumorigenicity protein 13) } \\
\text { (putative tumor suppressor st13) (protein } \\
\text { fam10a1) (progesterone receptor- } \\
\text { associate) }\end{array}$ & + & + & + & + & + & & $\mathrm{u}$ & & $\begin{array}{l}\text { Nsp12 } \\
\text { Orf3b } \\
\text { Orf6 } \\
\text { Orf8 } \\
\text { Orf10 }\end{array}$ & & + & [273] \\
\hline 3 & STIP1 & Stress-induced-phosphoprotein 1 & & + & & & & & $\mathrm{u}$ & $d$ & $\begin{array}{l}\text { E } \\
\text { Nsp12 } \\
\text { Orf3a } \\
\text { Orf8 }\end{array}$ & & + & [14] \\
\hline 2 & STRBP & $\begin{array}{l}\text { Spermatid perinuclear RNA-binding } \\
\text { protein }\end{array}$ & + & & & & & & & & Nsp15 & & + & \\
\hline 4 & SUB1 & $\begin{array}{l}\text { Activated RNA polymerase II } \\
\text { transcriptional coactivator p15 (PC4, } \\
\text { RPO2TC1) }\end{array}$ & + & & + & & & + & $\mathrm{u}$ & $d$ & & + & + & \\
\hline 2 & SUGT1 & $\begin{array}{l}\text { Protein SGT1 homolog (Suppressor of G2 } \\
\text { allele of SKP1 homolog) }\end{array}$ & & + & & & & & $\mathrm{u}$ & & $\begin{array}{l}\text { Nsp12 } \\
\text { Nsp15 }\end{array}$ & & + & \\
\hline 2 & SUMO1 & Small ubiquitin-related modifier & + & & & & & & & $d$ & & & + & [274] \\
\hline 9 & SUPT16H & FACT complex subunit SPT16 & + & & + & + & & & & $d$ & & + & & \\
\hline 2 & SUPT5H & Transcription elongation factor SPT5 & & & & + & & & & & & + & & \\
\hline 2 & SWAP70 & Switch-associated protein 70 & & + & & & & & & d & Nsp2 & & + & \\
\hline 11 & TALDO1 & Transaldolase & & + & & + & & & $\mathrm{u}$ & $d$ & & & + & [275] \\
\hline 3 & TBCA & Tubulin-specific chaperone A & & + & & & & & & & Nsp11 & & + & \\
\hline 3 & TCL1A & T-cell leukemia/lymphoma protein $1 \mathrm{~A}$ & & + & & & & & $\mathrm{u}$ & $d$ & & & + & \\
\hline 7 & TCP1 & $\begin{array}{l}\text { T-complex protein } 1 \text { subunit alpha (tcp-1- } \\
\text { alpha) (cct-alpha) }\end{array}$ & & + & + & & + & & & $d$ & Orf10 & & + & [51] \\
\hline 4 & TEX10 & Testis-expressed protein 10 & & & & + & & & & & & + & & \\
\hline 3 & TFG & TRK-fused gene protein & + & & & + & & & & & & & + & \\
\hline 4 & TGM2 & $\begin{array}{l}\text { Protein-glutamine gamma- } \\
\text { glutamyltransferase } 2\end{array}$ & & & & + & & & $\mathrm{u}$ & $d$ & & & + & [276] \\
\hline 9 & THBS1 & Thrombospondin-1 & + & & & & & & $\mathrm{u}$ & $d$ & & & + & [277] \\
\hline 29 & TLN1 & Talin-1 & + & + & + & + & & & $\mathrm{u}$ & $d$ & & & + & [278] \\
\hline 4 & TLN2 & Talin-2 & + & & & & & & $\mathrm{u}$ & & & & + & \\
\hline 6 & TNC & Tenascin C & + & & & & & & & $d$ & & & + & [279] \\
\hline 5 & TNPO1 & Transportin-1, KPNB2 & & + & & & & & & & & & + & \\
\hline 3 & TOP1 & DNA topoisomerase $1(\mathrm{Scl} 70)$ & & + & + & + & & + & u & & & + & & [280] \\
\hline 5 & TP53I3 & Quinone oxidoreductase & & & & + & & & $\mathrm{u}$ & $d$ & & & + & \\
\hline 3 & TPD52L2 & Tumor protein D54 & + & & & & & & $\mathrm{u}$ & $d$ & $\begin{array}{l}\text { Nsp4 } \\
\text { Orf6 }\end{array}$ & & + & \\
\hline 2 & TPI1 & Triosephosphate isomerase & & & + & & & & & $d$ & Nsp15 & & + & {$[53]$} \\
\hline 16 & TPM1 & Tropomyosin 1 alpha chain & + & + & + & + & + & + & $\mathrm{u}$ & $d$ & Nsp9 & & + & [281] \\
\hline 17 & TPM2 & Tropomyosin beta chain & + & & + & + & & + & $\mathrm{u}$ & $d$ & & & + & [25] \\
\hline 6 & TPM3 & Tropomyosin alpha-3 chain & + & + & + & + & + & + & $\mathrm{u}$ & $d$ & & & + & [282] \\
\hline 20 & TPM4 & Tropomyosin alpha-4 chain & + & + & + & + & + & + & $\mathrm{u}$ & $d$ & & & + & [283] \\
\hline 2 & TPP1 & Tripeptidyl-peptidase 1 & + & & & & & & $\mathrm{u}$ & $d$ & & + & & \\
\hline 4 & TPP2 & Tripeptidyl-peptidase 2 & & + & & & & & & & & & + & \\
\hline 4 & TPR & Nucleoprotein TPR & + & & & & & & $\mathrm{u}$ & $\mathrm{d}$ & & & + & [284] \\
\hline 4 & TPT1 & Tumor protein, translationally-controlled & + & & & & & & $\mathrm{u}$ & d & & & + & \\
\hline
\end{tabular}


bioRxiv preprint doi: https://doi.org/10.1101/2021.07.30.454526; this version posted August 4, 2021. The copyright holder for this preprint (which was not certified by peer review) is the author/funder, who has granted bioRxiv a license to display the preprint in perpetuity. It is made available under aCC-BY 4.0 International license.

\begin{tabular}{|c|c|c|c|c|c|c|c|c|c|c|c|c|c|c|}
\hline 3 & TSN & Translin & & & & + & & & & d & & & + & \\
\hline 3 & TTLL12 & Tubulin-tyrosine ligase-like protein 12 & & + & + & & & & & d & & & + & [285] \\
\hline 2 & TTLL3 & Tubulin monoglycylase TTLL3 & & + & & & & & $\mathrm{u}$ & & & & + & \\
\hline 4 & TUBA1C & Tubulin alpha-1C, tubulin alpha-6 & + & + & + & + & + & + & $\mathrm{u}$ & d & & + & + & [286] \\
\hline 10 & TUBA3C & Tubulin alpha-3C chain, TUBA2 & & & + & & + & & & & & + & + & \\
\hline 12 & TUBA4A & Tubulin alpha-4A chain, TUBA1 & + & + & & + & & & $\mathrm{u}$ & $d$ & & + & + & [287] \\
\hline 7 & TUBB & Tubulin beta chain, TUBB5 & + & + & + & & + & & $\mathrm{u}$ & $d$ & & + & + & [288] \\
\hline 4 & TUBB1 & Tubulin beta- 1 chain & + & + & + & & & & & & & + & + & [289] \\
\hline 2 & TUBB4A & Tubulin beta-4A chain, TUBB4, TUBB5 & & + & & & & & $\mathrm{u}$ & $d$ & & & + & [290] \\
\hline 12 & TUBB4B & Tubulin beta-4B chain, TUBB2C & + & + & & + & & + & u & d & & + & + & [289] \\
\hline 2 & TXN & Thioredoxin & + & & & & & & u & d & & & + & [291] \\
\hline 2 & TXNDC17 & Thioredoxin domain-containing protein 17 & + & + & & & & & u & d & & & + & \\
\hline 4 & TXNDC5 & Thioredoxin domain-containing protein 5 & + & & & + & & & $\mathrm{u}$ & d & & & + & \\
\hline 2 & TXNL1 & $\begin{array}{l}\text { Thioredoxin-like protein } 1 \text { ( } 32 \mathrm{kda} \\
\text { thioredoxin-related protein) }\end{array}$ & & & + & & & & u & & & & + & \\
\hline 15 & TXNRD1 & Thioredoxin reductase 1 , cytoplasmic & + & + & & + & & & $\mathrm{u}$ & $d$ & & & + & [291] \\
\hline 2 & U2AF2 & Splicing factor U2AF 65 kDa subunit & & + & & & & & & d & & & + & $\begin{array}{r}\{\text { Imai, } \\
1993 \\
\# 256\}\end{array}$ \\
\hline 15 & UBA1 & Ubiquitin-like modifier-activating enzyme 1 & + & + & + & + & + & + & $\mathrm{u}$ & $\mathrm{d}$ & & & + & [292] \\
\hline 2 & UBA2 & $\begin{array}{l}\text { Ubiquitin-like } 1 \text {-activating enzyme e1b } \\
\text { (sumo-1-activating enzyme subunit 2) } \\
\text { (anthracycline-associated resistance arx) }\end{array}$ & & & + & & & & & $\mathrm{d}$ & Nsp7 & & + & \\
\hline 2 & UBA6 & Ubiquitin-like modifier-activating enzyme 6 & & + & & & & & & & Nsp7 & & + & \\
\hline 2 & UBE2K & Ubiquitin-conjugating enzyme E2 K & & + & & & & & & & & & + & \\
\hline 2 & UBLE1A & $\begin{array}{l}\text { Ubiquitin-like } 1 \text {-activating enzyme e1a } \\
\text { (SUMO-1-activating enzyme subunit 1), } \\
\text { SAE1 }\end{array}$ & + & & + & & & & $\mathrm{u}$ & $d$ & & & + & [274] \\
\hline 2 & UBTF & $\begin{array}{l}\text { Nucleolar transcription factor } 1 \text {, } \\
\text { autoantigen NOR-90 }\end{array}$ & & & & + & & & & $d$ & & + & & [293] \\
\hline 2 & UCHL1 & $\begin{array}{l}\text { Ubiquitin carboxyl-terminal hydrolase } \\
\text { isozyme L1 }\end{array}$ & + & & & + & & & u & $d$ & $\begin{array}{l}\text { Nsp7 } \\
\text { Orf3 }\end{array}$ & & + & [294] \\
\hline 5 & UGDH & UDP-glucose 6-dehydrogenase & & & & + & & & $\mathrm{u}$ & $d$ & & & + & \\
\hline 6 & UGGT1 & $\begin{array}{l}\text { UDP-glucose:glycoprotein } \\
\text { glucosyltransferase 1, UGCGL1 }\end{array}$ & + & & & & & & & $\mathrm{d}$ & $\begin{array}{l}\text { Orf3a } \\
\text { Orf7a }\end{array}$ & & + & \\
\hline 18 & UPF1 & Regulator of nonsense transcripts 1 & + & & & + & & & & d & $\mathrm{N}$ & + & & \\
\hline 3 & USP5 & $\begin{array}{l}\text { Ubiquitin carboxyl-terminal hydrolase } 5 \\
\text { (ubiquitin thioesterase 5) (ubiquitin- } \\
\text { specific-processing protease 5) } \\
\text { (deubiquitinating enzyme 5) (isopeptidase } \\
\text { T) }\end{array}$ & + & + & + & & & & u & $d$ & & & + & \\
\hline 2 & USP7 & $\begin{array}{l}\text { Ubiquitin carboxyl-terminal hydrolase } \\
\text { (Herpes virus associated) }\end{array}$ & & & & + & & & $\mathrm{u}$ & & $\begin{array}{l}\mathrm{E} \\
\mathrm{M} \\
\text { Nsp4 } \\
\text { Orf7a } \\
\text { Orf7b }\end{array}$ & + & & \\
\hline 2 & USP9X & $\begin{array}{l}\text { Ubiquitin specific protease } 9, \mathrm{X} \\
\text { chromosome }\end{array}$ & + & & & & & & u & $d$ & & + & & \\
\hline 3 & VARS1 & Valine-tRNA ligase & & + & & & & & & & & & + & \\
\hline 4 & VASN & Vasorin & + & & & & & & $\mathrm{u}$ & $d$ & & & + & \\
\hline 4 & VAT1 & $\begin{array}{l}\text { Synaptic vesicle membrane protein VAT-1 } \\
\text { homolog }\end{array}$ & + & & & & & & u & d & $\begin{array}{l}\text { Orf3b } \\
\text { Orf6 }\end{array}$ & & + & \\
\hline 27 & VCL & Vinculin & + & & & + & & & $\mathrm{u}$ & $d$ & Nsp14 & & + & [295] \\
\hline 18 & VCP & $\begin{array}{l}\text { Transitional endoplasmic reticulum } \\
\text { ATPase (Valosin-containing protein) (ER) }\end{array}$ & + & + & + & + & + & + & $\mathrm{u}$ & $d$ & & & + & [296] \\
\hline 17 & VIM & Vimentin & + & + & + & + & + & + & $\mathrm{u}$ & $d$ & & + & + & [297] \\
\hline 2 & VPS35 & Vacuolar protein sorting 35 & & & & & + & & $\mathrm{u}$ & d & & & + & [298] \\
\hline 6 & WARS & $\begin{array}{l}\text { Tryptophanyl-tRNA synthetase, } \\
\text { cytoplasmic }\end{array}$ & + & + & & & & & u & $d$ & & & + & [299] \\
\hline 5 & WDR18 & WD repeat-containing protein 18 & & & & + & & & & d & Nsp15 & + & & \\
\hline 2 & XPNPEP1 & Xaa-Pro aminopeptidase 1 & & + & + & & & & & d & & & + & \\
\hline 4 & XPO1 & Exportin-1 & & + & & & & & & & $\begin{array}{l}\text { Nsp4 } \\
\text { Orf7a }\end{array}$ & & + & \\
\hline 10 & XPO2 & Exportin-2, CAS, CSE1L & & + & & & & & & $d$ & & & + & \\
\hline 5 & XPOT & Exportin-T (trna exportin) (exportin(trna)) & & + & + & & & & $\mathrm{u}$ & & Orf7a & & + & \\
\hline 32 & XRCC5 & $\begin{array}{l}\text { ATP-dependent DNA helicase } 2 \text { subunit } 2 \text {, } \\
\text { Ku80 }\end{array}$ & + & + & + & + & + & + & & $d$ & & + & + & [300] \\
\hline
\end{tabular}


bioRxiv preprint doi: https://doi.org/10.1101/2021.07.30.454526; this version posted August 4, 2021. The copyright holder for this preprint (which was not certified by peer review) is the author/funder, who has granted bioRxiv a license to display the preprint in perpetuity. It is made available under aCC-BY 4.0 International license.

\begin{tabular}{|c|c|c|c|c|c|c|c|c|c|c|c|c|c|}
\hline 30 & XRCC6 & $\begin{array}{l}\text { ATP-dependent DNA helicase } 2 \text { subunit } 1 \text {, } \\
\text { Ku70 }\end{array}$ & + & + & + & + & + & + & $\mathrm{u}$ & d & + & + & [300] \\
\hline 6 & YARS & $\begin{array}{l}\text { Tyrosine-tRNA ligase, cytoplasmic, } \\
\text { YARS1 }\end{array}$ & & + & & & & & $\mathrm{u}$ & $d$ & & + & [301] \\
\hline 3 & YBX1 & Y-box-binding protein 1 & & & + & + & & & u & $d$ & + & & [302] \\
\hline 6 & YBX3 & Y-box-binding protein 3 & + & & + & + & & + & $\mathrm{u}$ & d & + & & [303] \\
\hline 12 & YWHAB & 14-3-3 protein beta/alpha & + & + & + & + & + & + & $\mathrm{u}$ & d & & + & \\
\hline 15 & YWHAE & 14-3-3 protein epsilon & + & + & + & + & + & + & $\mathrm{u}$ & $d$ & & + & [254] \\
\hline 6 & YWHAG & 14-3-3 protein gamma & + & + & + & + & + & + & u & $d$ & & + & [254] \\
\hline 5 & YWHAH & 14-3-3 protein eta & + & + & + & + & + & & & $d$ & & + & [304] \\
\hline 7 & YWHAQ & $14-3-3$ protein theta & + & + & + & + & + & + & $\mathrm{u}$ & $d$ & & + & [243] \\
\hline 7 & YWHAZ & 14-3-3 protein zeta/delta & + & + & + & + & + & + & $\mathrm{u}$ & $d$ & & + & [305] \\
\hline 2 & ZPR1 & Zinc finger protein ZPR1 & & + & & & & & $\mathrm{u}$ & d & & + & [306] \\
\hline
\end{tabular}

Columns from left to right: $\mathrm{P}$ (the largest number of peptides identified for a protein by mass spectrometry for all cell lines), gene symbol, protein name, cell lines (HFL1 fetal lung fibroblast, HS-Sultan B lymphoblast, Wil2-NS B-lymphoblast, A549 lung epithelial cell, Jurkat T-lymphoblast, HEp-2 fibroblast), SARS-Cov-2 infection (up-regulated, down-regulated, interactome of specific viral protein), dermatan sulfate (DS) affinity (high affinity: eluted from DS-affinity resins with $1.0 \mathrm{M} \mathrm{NaCl}$; low affinity: eluted with 0.4-0.6 M NaCl), Ref. (representive paper reporting autoantibodies specific for the autoAg protein). Highlighted in red: common (shared) autoAgs found in all 6 cell lines.

\section{References}

[1] R. D. Saunders, S. T. Nakajima, S. N. Rai, J. Pan, C. Gercel-Taylor, D. D. Taylor. Alterations in antibody subclass immune reactivity to trophoblast-derived fetal fibronectin and a2-macroglobulin in women with recurrent pregnancy loss. Am J Reprod Immunol, 2012;68:43849.

[2] C. C. Bunn, R. M. Bernstein, M. B. Mathews. Autoantibodies against alanyl-tRNA synthetase and tRNAAla coexist and are associated with myositis. The Journal of experimental medicine, 1986;163:1281-91.

[3] A. Petrohai, G. Nagy, S. Bosze, F. Hudecz, E. Zsiros, G. Paragh et al. Detection of citrate synthase-reacting autoantibodies after heart transplantation: an epitope mapping study. Transplant international : official journal of the European Society for Organ Transplantation, 2005;17:834-40.

[4] M. Capello, J. V. Vykoukal, H. Katayama, L. E. Bantis, H. Wang, D. L. Kundnani et al. Exosomes harbor B cell targets in pancreatic adenocarcinoma and exert decoy function against complement-mediated cytotoxicity. Nature communications, 2019;10:254.

[5] P. V. Mande, F. R. Parikh, I. Hinduja, K. Zaveri, R. Vaidya, R. Gajbhiye et al. Identification and validation of candidate biomarkers involved in human ovarian autoimmunity. Reprod Biomed Online, 2011;23:471-83.

[6] J. J. van Beers, C. M. Schwarte, J. Stammen-Vogelzangs, E. Oosterink, B. Bozic, G. J. Pruijn. The rheumatoid arthritis synovial fluid citrullinome reveals novel citrullinated epitopes in apolipoprotein $\mathrm{E}$, myeloid nuclear differentiation antigen, and beta-actin. Arthritis and rheumatism, 2013;65:69-80.

[7] D. Chatterjee, M. Pieroni, M. Fatah, F. Charpentier, K. S. Cunningham, D. A. Spears et al. An autoantibody profile detects Brugada syndrome and identifies abnormally expressed myocardial proteins. European heart journal, 2020;41:2878-90.

[8] E. Vainio, G. M. Lenoir, R. M. Franklin. Autoantibodies in three populations of Burkitt's lymphoma patients. Clinical and experimental immunology, 1983;54:387-96.

[9] C. Hanrotel-Saliou, I. Segalen, Y. Le Meur, P. Youinou, Y. Renaudineau. Glomerular antibodies in lupus nephritis. Clin Rev Allergy Immunol, 2011;40:151-8.

[10] C. P. Moritz, Y. Tholance, O. Stoevesandt, K. Ferraud, J. P. Camdessanché, J. C. Antoine. CIDP Antibodies Target Junction Proteins and Identify Patient Subgroups: An Autoantigenomic Approach. Neurology(R) neuroimmunology \& neuroinflammation, $2021 ; 8$.

[11] R. Rucksaken, O. Haonon, P. Pinlaor, C. Pairojkul, S. Roytrakul, P. Yongvanit et al. Plasma IgG autoantibody against actin-related protein 3 in liver fluke Opisthorchis viverrini infection. Parasite immunology, 2015;37:340-8.

[12] R. Bei, G. J. Mizejewski. Alpha-fetoprotein is an autoantigen in hepatocellular carcinoma and juvenile Batten disease. Frontiers in bioscience (Landmark edition), 2020;25:912-29.

[13] M. H. Rivner, B. M. Quarles, J. X. Pan, Z. Yu, J. F. Howard, Jr., A. Corse et al. Clinical features of LRP4/agrin-antibody-positive myasthenia gravis: A multicenter study. Muscle \& nerve, 2020;62:333-43.

[14] H. Maejima, R. Nagashio, K. Yanagita, Y. Hamada, Y. Amoh, Y. Sato et al. Moesin and stress-induced phosphoprotein-1 are possible serodiagnostic markers of psoriasis. PloS one, 2014;9:e101773.

[15] F. Sköldberg, L. Rönnblom, M. Thornemo, A. Lindahl, P. I. Bird, F. Rorsman et al. Identification of AHNAK as a novel autoantigen in systemic lupus erythematosus. Biochemical and biophysical research communications, 2002;291:951-8.

[16] S. Biswas, S. Sharma, A. Saroha, D. S. Bhakuni, R. Malhotra, M. Zahur et al. Identification of novel autoantigen in the synovial fluid of rheumatoid arthritis patients using an immunoproteomics approach. PloS one, 2013;8:e56246.

[17] M. Prunotto, M. L. Carnevali, G. Candiano, C. Murtas, M. Bruschi, E. Corradini et al. Autoimmunity in membranous nephropathy targets aldose reductase and SOD2. Journal of the American Society of Nephrology : JASN, 2010;21:507-19.

[18] J. Nehring, L. A. Schirmbeck, J. Friebus-Kardash, D. Dubler, U. Huynh-Do, C. Chizzolini et al. Autoantibodies Against Albumin in Patients With Systemic Lupus Erythematosus. Frontiers in immunology, 2018;9:2090.

[19] S. L. Edassery, S. V. Shatavi, J. P. Kunkel, C. Hauer, C. Brucker, K. Penumatsa et al. Autoantigens in ovarian autoimmunity associated with unexplained infertility and premature ovarian failure. Fertility and sterility, 2010;94:2636-41.

[20] K. C. Cheng, Y. J. Wu, K. H. Cheng, K. Y. Cheng, K. J. Chen, W. C. Wu et al. Autoantibody against aldehyde dehydrogenase 2 could be a biomarker to monitor progression of Graves' orbitopathy. Graefe's archive for clinical and experimental ophthalmology = Albrecht von Graefes Archiv fur klinische und experimentelle Ophthalmologie, 2018;256:1195-201. 
bioRxiv preprint doi: https://doi.org/10.1101/2021.07.30.454526; this version posted August 4, 2021. The copyright holder for this preprint (which was not certified by peer review) is the author/funder, who has granted bioRxiv a license to display the preprint in perpetuity. It is made available under aCC-BY 4.0 International license.

[21] D. Privitera, V. Corti, M. Alessio, M. A. Volontè, V. Lampasona, G. Comi et al. Proteomic identification of aldolase A as an autoantibody target in patients with atypical movement disorders. Neurological sciences : official journal of the Italian Neurological Society and of the Italian Society of Clinical Neurophysiology, 2013;34:313-20.

[22] K. Morohoshi, M. Ohbayashi, N. Patel, V. Chong, A. C. Bird, S. J. Ono. Identification of anti-retinal antibodies in patients with age-related macular degeneration. Experimental and molecular pathology, 2012;93:193-9.

[23] D. Lu, E. Kuhn, R. E. Bristow, R. L. Giuntoli, 2nd, S. K. Kjær, M. Shih le et al. Comparison of candidate serologic markers for type I and type II ovarian cancer. Gynecol Oncol, 2011;122:560-6.

[24] M. C. Pott, N. Frede, J. Wanders, L. Hammarström, E. O. Glocker, C. Glocker et al. Autoantibodies against BAFF, APRIL or IL21 - an alternative pathogenesis for antibody-deficiencies? BMC immunology, 2017;18:34.

[25] Q. Yang, M. H. Roehrl, J. Y. Wang. Proteomic profiling of antibody-inducing immunogens in tumor tissue identifies PSMA1, LAP3, ANXA3, and maspin as colon cancer markers. Oncotarget, 2018;9:3996-4019.

[26] D. J. Caster, E. A. Korte, M. L. Merchant, J. B. Klein, D. W. Wilkey, B. H. Rovin et al. Autoantibodies targeting glomerular annexin A2 identify patients with proliferative lupus nephritis. Proteomics Clin Appl, 2015;9:1012-20.

[27] A. Satoh, K. Suzuki, E. Takayama, K. Kojima, T. Hidaka, M. Kawakami et al. Detection of anti-annexin IV and V antibodies in patients with antiphospholipid syndrome and systemic lupus erythematosus. The Journal of rheumatology, 1999;26:1715-20.

[28] A. Hrycek, P. Cieślik. Annexin A5 and anti-annexin antibodies in patients with systemic lupus erythematosus. Rheumatol Int, 2012;32:133542.

[29] Y. Seko, A. Matsumoto, T. Fukuda, Y. Imai, T. Fujimura, H. Taka et al. A case of neonatal lupus erythematosus presenting delayed dilated cardiomyopathy with circulating autoantibody to annexin A6. Int Heart J, 2007;48:407-15.

[30] S. Jarius, B. Wildemann. 'Medusa head ataxia': the expanding spectrum of Purkinje cell antibodies in autoimmune cerebellar ataxia. Part 3: Anti-Yo/CDR2, anti-Nb/AP3B2, PCA-2, anti-Tr/DNER, other antibodies, diagnostic pitfalls, summary and outlook. Journal of neuroinflammation, 2015;12:168.

[31] Y. Katsumata, Y. Kawaguchi, S. Baba, S. Hattori, K. Tahara, K. Ito et al. Identification of three new autoantibodies associated with systemic lupus erythematosus using two proteomic approaches. Molecular \& cellular proteomics : MCP, 2011;10:M110.005330.

[32] N. Vuilleumier, F. Montecucco, O. Hartley. Autoantibodies to apolipoprotein A-1 as a biomarker of cardiovascular autoimmunity. World J Cardiol, 2014;6:314-26.

[33] E. G. Kamburova, M. L. Gruijters, T. Kardol-Hoefnagel, B. W. Wisse, I. Joosten, W. A. Allebes et al. Antibodies against ARHGDIB are associated with long-term kidney graft loss. American journal of transplantation : official journal of the American Society of Transplantation and the American Society of Transplant Surgeons, 2019;19:3335-44.

[34] C. K. Heo, H. M. Hwang, W. H. Lim, H. J. Lee, J. S. Yoo, K. J. Lim et al. Cyclic Peptide Mimotopes for the Detection of Serum Anti-ATIC Autoantibody Biomarker in Hepato-Cellular Carcinoma. International journal of molecular sciences, 2020;21.

[35] B. Krishnan, C. Massilamany, R. H. Basavalingappa, A. Gangaplara, R. A. Rajasekaran, M. Z. Afzal et al. Epitope Mapping of SERCA2a Identifies an Antigenic Determinant That Induces Mainly Atrial Myocarditis in A/J Mice. Journal of immunology (Baltimore, Md : 1950), 2018;200:523-37.

[36] J. Creaney, I. M. Dick, D. Yeoman, S. Wong, B. W. Robinson. Auto-antibodies to $\beta$-F1-ATPase and vimentin in malignant mesothelioma. PloS one, 2011;6:e26515.

[37] A. Polgár, A. Falus, E. Koó, I. Ujfalussy, M. Seszták, I. Szuts et al. Elevated levels of synovial fluid antibodies reactive with the small proteoglycans biglycan and decorin in patients with rheumatoid arthritis or other joint diseases. Rheumatology (Oxford, England), 2003;42:522-7.

[38] N. M. Bhat, C. M. Adams, Y. Chen, M. M. Bieber, N. N. Teng. Identification of Cell Surface Straight Chain Poly-N-Acetyl-Lactosamine Bearing Protein Ligands for VH4-34-Encoded Natural IgM Antibodies. Journal of immunology (Baltimore, Md : 1950), 2015;195:5178-88.

[39] V. M. Beutgen, C. Schmelter, N. Pfeiffer, F. H. Grus. Autoantigens in the trabecular meshwork and glaucoma-specific alterations in the natural autoantibody repertoire. Clinical \& translational immunology, 2020;9:e01101.

[40] L. Xu, J. R. Lee, S. Hao, X. B. Ling, J. D. Brooks, S. X. Wang et al. Improved detection of prostate cancer using a magneto-nanosensor assay for serum circulating autoantibodies. PloS one, 2019;14:e0221051.

[41] C. N. Gruber, R. S. Patel, R. Trachtman, L. Lepow, F. Amanat, F. Krammer et al. Mapping Systemic Inflammation and Antibody Responses in Multisystem Inflammatory Syndrome in Children (MIS-C). Cell, 2020;183:982-95.e14.

[42] Y. Ikeda, G. Toda, N. Hashimoto, T. Maruyama, H. Oka. Antibody that recognizes conformations of calmodulin in the serum from patient with chronic active hepatitis. Biochemical and biophysical research communications, 1987;144:191-7.

[43] P. Eggleton, F. J. Ward, S. Johnson, M. A. Khamashta, G. R. Hughes, V. A. Hajela et al. Fine specificity of autoantibodies to calreticulin: epitope mapping and characterization. Clinical and experimental immunology, 2000;120:384-91.

[44] B. Terrier, M. C. Tamby, L. Camoin, P. Guilpain, C. Broussard, G. Bussone et al. Identification of target antigens of antifibroblast antibodies in pulmonary arterial hypertension. American journal of respiratory and critical care medicine, 2008;177:1128-34.

[45] C. K. Weber, M. Haslbeck, M. Englbrecht, B. Sehnert, D. Mielenz, D. Graef et al. Antibodies to the endoplasmic reticulum-resident chaperones calnexin, BiP and Grp94 in patients with rheumatoid arthritis and systemic lupus erythematosus. Rheumatology (Oxford, England), 2010;49:2255-63.

[46] K. Matsuo, Y. Xiang, H. Nakamura, K. Masuko, K. Yudoh, K. Noyori et al. Identification of novel citrullinated autoantigens of synovium in rheumatoid arthritis using a proteomic approach. Arthritis research \& therapy, 2006;8:R175.

[47] W. H. Li, J. Zhao, H. Y. Li, H. Liu, A. L. Li, H. X. Wang et al. Proteomics-based identification of autoantibodies in the sera of healthy Chinese individuals from Beijing. Proteomics, 2006;6:4781-9.

[48] X. L. Wang, T. Y. Ling, M. C. Charlesworth, J. J. Figueroa, P. Low, W. K. Shen et al. Autoimmunoreactive IgGs against cardiac lipid raftassociated proteins in patients with postural orthostatic tachycardia syndrome. Translational research : the journal of laboratory and clinical medicine, 2013;162:34-44.

[49] K. Furuta, B. Hildebrandt, S. Matsuoka, K. Kiyosawa, G. Reimer, C. Luderschmidt et al. Immunological characterization of heterochromatin protein p25beta autoantibodies and relationship with centromere autoantibodies and pulmonary fibrosis in systemic scleroderma. $\mathrm{J}$ Mol Med (Berl), 1998;76:54-60.

[50] K. Ohyama, M. Baba, M. Tamai, N. Aibara, K. Ichinose, N. Kishikawa et al. Proteomic profiling of antigens in circulating immune complexes associated with each of seven autoimmune diseases. Clin Biochem, 2015;48:181-5.

[51] S. I. Yokota, D. Hirata, S. Minota, T. Higashiyama, M. Kurimoto, H. Yanagi et al. Autoantibodies against chaperonin CCT in human sera with rheumatic autoimmune diseases: comparison with antibodies against other Hsp60 family proteins. Cell Stress Chaperones, 2000;5:337-46. 
bioRxiv preprint doi: https://doi.org/10.1101/2021.07.30.454526; this version posted August 4, 2021. The copyright holder for this preprint (which was not certified by peer review) is the author/funder, who has granted bioRxiv a license to display the preprint in perpetuity. It is made available under aCC-BY 4.0 International license.

[52] K. Hirai, H. Maeda, K. Omori, T. Yamamoto, S. Kokeguchi, S. Takashiba. Serum antibody response to group II chaperonin from Methanobrevibacter oralis and human chaperonin CCT. Pathog Dis, 2013;68:12-9.

[53] G. Bussone, H. Dib, M. C. Tamby, C. Broussard, C. Federici, G. Woimant et al. Identification of new autoantibody specificities directed at proteins involved in the transforming growth factor beta pathway in patients with systemic sclerosis. Arthritis research \& therapy, 2011;13:R74.

[54] M. Ebrahimi, E. Nylander, B. Bäcklund, Y. B. Wahlin, P. J. Coates, K. Nylander. The use of a novel ELISA method for detection of antibodies against p63 in sera from patients diagnosed with oral and/or genital and skin lichen planus. Journal of oral pathology \& medicine : official publication of the International Association of Oral Pathologists and the American Academy of Oral Pathology, 2010;39:486-90.

[55] L. Zhu, W. Shen, M. Zhu, N. J. Coorey, A. P. Nguyen, D. Barthelmes et al. Anti-retinal antibodies in patients with macular telangiectasia type 2. Investigative ophthalmology \& visual science, 2013;54:5675-83.

[56] M. Goto, K. Kuribayashi, Y. Takahashi, T. Kondoh, M. Tanaka, D. Kobayashi et al. Identification of autoantibodies expressed in acquired aplastic anaemia. British journal of haematology, 2013;160:359-62.

[57] J. H. Rho, W. Zhang, M. Murali, M. H. Roehrl, J. Y. Wang. Human proteins with affinity for dermatan sulfate have the propensity to become autoantigens. Am J Pathol, 2011;178:2177-90.

[58] M. K. Koivula, S. Aman, A. Karjalainen, M. Hakala, J. Risteli. Are there autoantibodies reacting against citrullinated peptides derived from type I and type II collagens in patients with rheumatoid arthritis? Annals of the rheumatic diseases, 2005;64:1443-50.

[59] J. Pardos-Gea, J. Cortés-Hernández, J. Castro-Marrero, E. Balada, J. Ordi-Ros. Autoantibodies to types I and IV collagen and heart valve disease in systemic lupus erythematosus/antiphospholipid syndrome. Clinical rheumatology, 2017;36:1401-6.

[60] G. R. Araujo, J. E. Fonseca, P. T. Fujimura, J. P. Cunha-Junior, C. H. Silva, A. F. Mourão et al. Anti-type II collagen antibodies detection and avidity in patients with oligoarticular and polyarticular forms of juvenile idiopathic arthritis. Immunology letters, 2015;165:20-5.

[61] G. Nakos, A. Adams, N. Andriopoulos. Antibodies to collagen in patients with idiopathic pulmonary fibrosis. Chest, 1993;103:1051-8.

[62] R. R. Hachem, V. Tiriveedhi, G. A. Patterson, A. Aloush, E. P. Trulock, T. Mohanakumar. Antibodies to K-a 1 tubulin and collagen V are associated with chronic rejection after lung transplantation. American journal of transplantation : official journal of the American Society of Transplantation and the American Society of Transplant Surgeons, 2012;12:2164-71.

[63] D. S. Nath, H. I. Basha, T. Mohanakumar. Antihuman leukocyte antigen antibody-induced autoimmunity: role in chronic rejection. Current opinion in organ transplantation, 2010;15:16-20.

[64] T. J. Vece, L. B. Watkin, S. Nicholas, D. Canter, M. C. Braun, R. P. Guillerman et al. Copa Syndrome: a Novel Autosomal Dominant Immune Dysregulatory Disease. J Clin Immunol, 2016;36:377-87.

[65] Q. Yang, J. Qin, G. Sun, C. Qiu, D. Jiang, H. Ye et al. Discovery and Validation of Serum Autoantibodies Against Tumor-Associated Antigens as Biomarkers in Gastric Adenocarcinoma Based on the Focused Protein Arrays. Clin Transl Gastroenterol, 2020;12:e00284.

[66] H. S. Hong, W. H. Chung, S. I. Hung, M. J. Chen, S. H. Lee, L. C. Yang. Clinical association of anti-golgi autoantibodies and their autoantigens. Scand J Immunol, 2004;59:79-87.

[67] M. Rouwette, J. P. Noben, J. Van Horssen, B. Van Wijmeersch, R. Hupperts, P. J. Jongen et al. Identification of coronin-1a as a novel antibody target for clinically isolated syndrome and multiple sclerosis. Journal of neurochemistry, 2013;126:483-92.

[68] C. Bénistant, J. F. Bourgaux, H. Chapuis, N. Mottet, S. Roche, J. P. Bali. The COOH-terminal Src kinase Csk is a tumor antigen in human carcinoma. Cancer research, 2001;61:1415-20.

[69] R. Dummer, A. Mittelman, F. P. Fanizzi, G. Lucchese, J. Willers, D. Kanduc. Non-self-discrimination as a driving concept in the identification of an immunodominant HMW-MAA epitopic peptide sequence by autoantibodies from melanoma cancer patients. Int $\mathrm{J}$ Cancer, 2004;111:720-6.

[70] V. Vetvicka, M. Fusek. Cathepsin D: Autoantibody profiling as a diagnostic marker for cancers. World J Clin Oncol, 2013;4:1-3.

[71] F. Schneider, R. Aggarwal, D. Bi, K. Gibson, C. Oddis, S. A. Yousem. The pulmonary histopathology of anti-KS transfer RNA synthetase syndrome. Arch Pathol Lab Med, 2015;139:122-5.

[72] J. Pitsch, D. Kamalizade, A. Braun, J. C. Kuehn, P. E. Gulakova, T. Rüber et al. Drebrin Autoantibodies in Patients with Seizures and Suspected Encephalitis. Ann Neurol, 2020;87:869-84.

[73] C. A. Brandsma, H. A. Kerstjens, W. H. van Geffen, M. Geerlings, D. S. Postma, M. N. Hylkema et al. Differential switching to IgG and IgA in active smoking COPD patients and healthy controls. Eur Respir J, 2012;40:313-21.

[74] M. J. Fritzler, J. C. Hamel, R. L. Ochs, E. K. Chan. Molecular characterization of two human autoantigens: unique cDNAs encoding 95- and 160-kD proteins of a putative family in the Golgi complex. The Journal of experimental medicine, 1993;178:49-62.

[75] M. C. Garcia, J. Zhou, D. Henning, F. C. Arnett, B. C. Valdez. Unique epitopes in RNA helicase II/Gu protein recognized by serum from a watermelon stomach patient. Molecular immunology, 2000;37:351-9.

[76] H. J. Netter, H. Will, C. Szostecki, H. H. Guldner. Repetitive P68-autoantigen specific epitopes recognized by human anti-(U1) small nuclear ribonucleoprotein autoantibodies. Journal of autoimmunity, 1991;4:651-63.

[77] R. H. Scofield. Do we need new autoantibodies in lupus? Arthritis research \& therapy, 2010;12:120.

[78] M. Vazquez-Del Mercado, C. A. Palafox-Sanchez, J. F. Munoz-Valle, G. Orozco-Barocio, E. Oregon-Romero, R. E. Navarro-Hernandez et al. High prevalence of autoantibodies to RNA helicase A in Mexican patients with systemic lupus erythematosus. Arthritis research \& therapy, 2010;12:R6.

[79] K. Yoneyama, R. Shibata, A. Igarashi, S. Kojima, Y. Kodani, K. Nagata et al. Proteomic identification of dihydrolipoamide dehydrogenase as a target of autoantibodies in patients with endometrial cancer. Anticancer research, 2014;34:5021-7.

[80] D. R. Fregeau, T. Prindiville, R. L. Coppel, M. Kaplan, E. R. Dickson, M. E. Gershwin. Inhibition of alpha-ketoglutarate dehydrogenase activity by a distinct population of autoantibodies recognizing dihydrolipoamide succinyltransferase in primary biliary cirrhosis. Hepatology, 1990;11:975-81.

[81] M. Oka, S. Sato, H. Soda, M. Fukuda, S. Kawabata, K. Nakatomi et al. Autoantibody to heat shock protein Hsp40 in sera of lung cancer patients. Japanese journal of cancer research : Gann, 2001;92:316-20.

[82] M. M. Harper, D. Rudd, K. J. Meyer, A. G. Kanthasamy, V. Anantharam, A. A. Pieper et al. Identification of chronic brain protein changes and protein targets of serum auto-antibodies after blast-mediated traumatic brain injury. Heliyon, 2020;6:e03374.

[83] L. Dai, J. Li, J. J. Tsay, T. A. Yie, J. S. Munger, H. Pass et al. Identification of autoantibodies to ECH1 and HNRNPA2B1 as potential biomarkers in the early detection of lung cancer. Oncoimmunology, 2017;6:e1310359.

[84] E. G. Kim, S. H. Kwak, D. Hwang, E. C. Yi, K. S. Park, B. K. Koo et al. The Level of Autoantibodies Targeting Eukaryote Translation Elongation Factor $1 \alpha 1$ and Ubiquitin-Conjugating Enzyme 2L3 in Nondiabetic Young Adults. Diabetes Metab J, 2016;40:154-60. 
bioRxiv preprint doi: https://doi.org/10.1101/2021.07.30.454526; this version posted August 4, 2021. The copyright holder for this preprint (which was not certified by peer review) is the author/funder, who has granted bioRxiv a license to display the preprint in perpetuity. It is made available under aCC-BY 4.0 International license.

[85] C. J. Mooney, E. J. Dunphy, B. Stone, D. G. McNeel. Identification of autoantibodies elicited in a patient with prostate cancer presenting as dermatomyositis. Int J Urol, 2006;13:211-7.

[86] K. Ohkouchi, H. Mizutani, M. Tanaka, M. Takahashi, K. Nakashima, M. Shimizu. Anti-elongation factor-1alpha autoantibody in adult atopic dermatitis patients. Int Immunol, 1999;11:1635-40.

[87] F. Fernández-Madrid, N. Tang, H. Alansari, J. L. Granda, L. Tait, K. C. Amirikia et al. Autoantibodies to Annexin XI-A and Other Autoantigens in the Diagnosis of Breast Cancer. Cancer research, 2004;64:5089-96.

[88] M. Bach, G. Winkelmann, R. Luhrmann. 20S small nuclear ribonucleoprotein U5 shows a surprisingly complex protein composition. Proceedings of the National Academy of Sciences of the United States of America, 1989;86:6038-42.

[89] E. A. Waterman, D. J. Gawkrodger, P. F. Watson, A. P. Weetman, E. H. Kemp. Autoantigens in vitiligo identified by the serological selection of a phage-displayed melanocyte cDNA expression library. The Journal of investigative dermatology, 2010;130:230-40.

[90] C. K. Heo, H. M. Hwang, H. J. Lee, S. S. Kwak, J. S. Yoo, D. Y. Yu et al. Serum anti-EIF3A autoantibody as a potential diagnostic marker for hepatocellular carcinoma. Sci Rep, 2019;9:11059.

[91] X. Chen, S. Fu, F. Chen, H. Chen, Z. Chen. Identification of tumor-associated antigens in human hepatocellular carcinoma by autoantibodies. Oncol Rep, 2008;20:979-85.

[92] Z. Betteridge, H. Chinoy, J. Vencovsky, J. Winer, K. Putchakayala, P. Ho et al. Identification of a novel autoantigen eukaryotic initiation factor 3 associated with polymyositis. Rheumatology (Oxford, England), 2020;59:1026-30.

[93] J. A. Luna Coronell, K. Sergelen, P. Hofer, I. Gyurján, S. Brezina, P. Hettegger et al. The Immunome of Colon Cancer: Functional In Silico Analysis of Antigenic Proteins Deduced from IgG Microarray Profiling. Genomics, proteomics \& bioinformatics, 2018;16:73-84.

[94] G. Suwarnalata, A. H. Tan, H. Isa, R. Gudimella, A. Anwar, M. F. Loke et al. Augmentation of Autoantibodies by Helicobacter pylori in Parkinson's Disease Patients May Be Linked to Greater Severity. PloS one, 2016;11:e0153725.

[95] C. Pagaza-Straffon, L. A. Marchat, L. Herrera, J. Díaz-Chávez, M. G. Avante, Y. P. Rodríguez et al. Evaluation of a panel of tumor-associated antigens in breast cancer. Cancer biomarkers : section A of Disease markers, 2020;27:207-11.

[96] L. B. Nabors, H. M. Furneaux, P. H. King. HuR, a novel target of anti-Hu antibodies, is expressed in non-neural tissues. Journal of neuroimmunology, 1998;92:152-9.

[97] S. Moscato, F. Pratesi, A. Sabbatini, D. Chimenti, M. Scavuzzo, R. Passatino et al. Surface expression of a glycolytic enzyme, alpha-enolase, recognized by autoantibodies in connective tissue disorders. Eur J Immunol, 2000;30:3575-84.

[98] D. T. O'Dwyer, V. Clifton, A. Hall, R. Smith, P. J. Robinson, P. A. Crock. Pituitary autoantibodies in lymphocytic hypophysitis target both gamma- and alpha-Enolase - a link with pregnancy? Archives of physiology and biochemistry, 2002;110:94-8.

[99] T. Akatsuka, N. Kobayashi, T. Ishikawa, T. Saito, M. Shindo, M. Yamauchi et al. Autoantibody response to microsomal epoxide hydrolase in hepatitis C and A. Journal of autoimmunity, 2007;28:7-18.

[100] I. N. Targoff, E. P. Trieu, F. W. Miller. Reaction of anti-OJ autoantibodies with components of the multi-enzyme complex of aminoacyl-tRNA synthetases in addition to isoleucyl-tRNA synthetase. The Journal of clinical investigation, 1993;91:2556-64.

[101] M. Garranzo-Asensio, P. San Segundo-Acosta, C. Povés, M. J. Fernández-Aceñero, J. Martínez-Useros, A. Montero-Calle et al. Identification of tumor-associated antigens with diagnostic ability of colorectal cancer by in-depth immunomic and seroproteomic analysis. Journal of proteomics, 2020;214:103635.

[102] C. Leveque, T. Hoshino, P. David, Y. Shoji-Kasai, K. Leys, A. Omori et al. The synaptic vesicle protein synaptotagmin associates with calcium channels and is a putative Lambert-Eaton myasthenic syndrome antigen. Proceedings of the National Academy of Sciences of the United States of America, 1992;89:3625-9.

[103] M. Capello, P. Cappello, F. C. Linty, R. Chiarle, I. Sperduti, A. Novarino et al. Autoantibodies to Ezrin are an early sign of pancreatic cancer in humans and in genetically engineered mouse models. Journal of hematology \& oncology, 2013;6:67.

[104] Z. Betteridge, H. Gunawardena, J. North, J. Slinn, N. McHugh. Anti-synthetase syndrome: a new autoantibody to phenylalanyl transfer RNA synthetase (anti-Zo) associated with polymyositis and interstitial pneumonia. Rheumatology (Oxford, England), 2007;46:1005-8.

[105] C. K. Heo, M. K. Woo, D. Y. Yu, J. Y. Lee, J. S. Yoo, H. S. Yoo et al. Identification of autoantibody against fatty acid synthase in hepatocellular carcinoma mouse model and its application to diagnosis of HCC. Int J Oncol, 2010;36:1453-9.

[106] S. Forti, M. J. Scanlan, A. Invernizzi, F. Castiglioni, S. Pupa, R. Agresti et al. Identification of breast cancer-restricted antigens by antibody screening of SKBR3 cDNA library using a preselected patient's serum. Breast cancer research and treatment, 2002;73:245-56.

[107] C. Desmetz, C. Bascoul-Mollevi, P. Rochaix, P. J. Lamy, A. Kramar, P. Rouanet et al. Identification of a new panel of serum autoantibodies associated with the presence of in situ carcinoma of the breast in younger women. Clinical cancer research : an official journal of the American Association for Cancer Research, 2009;15:4733-41.

[108] J. Kamhieh-Milz, V. Sterzer, H. Celik, O. Khorramshahi, R. Fadl Hassan Moftah, A. Salama. Identification of novel autoantigens via mass spectroscopy-based antibody-mediated identification of autoantigens (MS-AMIDA) using immune thrombocytopenic purpura (ITP) as a model disease. Journal of proteomics, 2017;157:59-70.

[109] M. Adachi-Hayama, A. Adachi, N. Shinozaki, T. Matsutani, T. Hiwasa, M. Takiguchi et al. Circulating anti-filamin C autoantibody as a potential serum biomarker for low-grade gliomas. BMC Cancer, 2014;14:452.

[110] W. Y. Wang, C. W. Twu, Y. C. Liu, H. H. Lin, C. J. Chen, J. C. Lin. Fibronectin promotes nasopharyngeal cancer cell motility and proliferation. Biomed Pharmacother, 2019;109:1772-84.

[111] X. Dong, M. Yang, H. Sun, J. Lü, Z. Zheng, Z. Li et al. Combined measurement of CA 15-3 with novel autoantibodies improves diagnostic accuracy for breast cancer. Onco Targets Ther, 2013;6:273-9.

[112] A. Regent, H. Dib, K. H. Ly, C. Agard, M. C. Tamby, N. Tamas et al. Identification of target antigens of anti-endothelial cell and anti-vascular smooth muscle cell antibodies in patients with giant cell arteritis: a proteomic approach. Arthritis research \& therapy, 2011;13:R107.

[113] Y. Kit, M. Starykovych, M. Vajrychova, J. Lenco, D. Zastavna, R. Stoika. Detection of novel auto-antigens in patients with recurrent miscarriage: description of an approach and preliminary findings. Croat Med J, 2014;55:259-64.

[114] F. Delunardo, D. Soldati, V. Bellisario, A. Berry, S. Camerini, M. Crescenzi et al. Anti-GAPDH Autoantibodies as a Pathogenic Determinant and Potential Biomarker of Neuropsychiatric Diseases. Arthritis \& rheumatology (Hoboken, NJ), 2016;68:2708-16.

[115] F. Schneider, S. A. Yousem, D. Bi, K. F. Gibson, C. V. Oddis, R. Aggarwal. Pulmonary pathologic manifestations of anti-glycyl-tRNA synthetase (anti-EJ)-related inflammatory myopathy. Journal of clinical pathology, 2014;67:678-83.

[116] A. Kiyota, S. Iwama, Y. Sugimura, S. Takeuchi, H. Takagi, N. Iwata et al. Identification of the novel autoantigen candidate Rab GDP dissociation inhibitor alpha in isolated adrenocorticotropin deficiency. Endocrine journal, 2015;62:153-60.

[117] O. Massa, M. Alessio, L. Russo, G. Nardo, V. Bonetto, F. Bertuzzi et al. Serological Proteome Analysis (SERPA) as a tool for the identification of new candidate autoantigens in type 1 diabetes. Journal of proteomics, 2013;82:263-73. 
bioRxiv preprint doi: https://doi.org/10.1101/2021.07.30.454526; this version posted August 4, 2021. The copyright holder for this preprint (which was not certified by peer review) is the author/funder, who has granted bioRxiv a license to display the preprint in perpetuity. It is made available under aCC-BY 4.0 International license.

[118] L. Carlsson, G. Ronquist, B. O. Nilsson, A. Larsson. Dominant prostasome immunogens for sperm-agglutinating autoantibodies of infertile men. Journal of andrology, 2004;25:699-705.

[119] J. M. Chung, Y. Jung, Y. P. Kim, J. Song, S. Kim, J. Y. Kim et al. Identification of the Thioredoxin-Like 2 Autoantibody as a Specific Biomarker for Triple-Negative Breast Cancer. Journal of breast cancer, 2018;21:87-90.

[120] P. V. Belousov, M. A. Afanasyeva, E. O. Gubernatorova, A. V. Bogolyubova, A. N. Uvarova, L. V. Putlyaeva et al. Multi-dimensional immunoproteomics coupled with in vitro recapitulation of oncogenic NRAS(Q61R) identifies diagnostically relevant autoantibody biomarkers in thyroid neoplasia. Cancer letters, 2019;467:96-106.

[121] Y. Muraki, I. Matsumoto, Y. Chino, T. Hayashi, E. Suzuki, D. Goto et al. Glucose-6-phosphate isomerase variants play a key role in the generation of anti-GPI antibodies: possible mechanism of autoantibody production. Biochemical and biophysical research communications, 2004;323:518-22.

[122] J. Yang, G. Tezel, R. V. Patil, C. Romano, M. B. Wax. Serum autoantibody against glutathione S-transferase in patients with glaucoma. Investigative ophthalmology \& visual science, 2001;42:1273-6.

[123] C. Stemmer, N. Tuaillon, A. M. Prieur, S. Muller. Mapping of B-cell epitopes recognized by antibodies to histones in subsets of juvenile chronic arthritis. Clinical immunology and immunopathology, 1995;76:82-9.

[124] J. Wesierska-Gadek, E. Penner, H. Lindner, E. Hitchman, G. Sauermann. Autoantibodies against different histone H1 subtypes in systemic lupus erythematosus sera. Arthritis and rheumatism, 1990;33:1273-8.

[125] Y. S. Kwon, J. Chung, G. T. Shin, S. Y. Lee, Y. J. Jang. Variable region genes of human monoclonal autoantibodies to histones H2A and H2B from a systemic lupus erythematosus patient. Molecular immunology, 2005;42:311-7.

[126] S. Vordenbaumen, P. Bohmer, R. Brinks, R. Fischer-Betz, J. Richter, E. Bleck et al. High diagnostic accuracy of histone H4-IgG autoantibodies in systemic lupus erythematosus. Rheumatology (Oxford, England), 2018;57:533-7.

[127] R. L. Rubin, S. A. Bell, R. W. Burlingame. Autoantibodies associated with lupus induced by diverse drugs target a similar epitope in the (H2A-H2B)-DNA complex. The Journal of clinical investigation, 1992;90:165-73.

[128] R. W. Burlingame, M. L. Boey, G. Starkebaum, R. L. Rubin. The central role of chromatin in autoimmune responses to histones and DNA in systemic lupus erythematosus. The Journal of clinical investigation, 1994;94:184-92.

[129] S. V. Baranova, P. S. Dmitrienok, N. V. Ivanisenko, V. N. Buneva, G. A. Nevinsky. Antibodies to H2a and H2b histones from the sera of HIVinfected patients catalyze site-specific degradation of these histones. Molecular bioSystems, 2017;13:1090-101.

[130] C. C. van Bavel, J. Dieker, S. Muller, J. P. Briand, M. Monestier, J. H. Berden et al. Apoptosis-associated acetylation on histone H2B is an epitope for lupus autoantibodies. Molecular immunology, 2009;47:511-6.

[131] J. Dieker, J. H. Berden, M. Bakker, J. P. Briand, S. Muller, R. Voll et al. Autoantibodies against Modified Histone Peptides in SLE Patients Are Associated with Disease Activity and Lupus Nephritis. PloS one, 2016;11:e0165373.

[132] S. V. Baranova, P. S. Dmitrenok, A. D. Zubkova, N. V. Ivanisenko, E. S. Odintsova, V. N. Buneva et al. Antibodies against H3 and H4 histones from the sera of HIV-infected patients catalyze site-specific degradation of these histones. Journal of molecular recognition : JMR, 2018;31:e2703.

[133] M. Bruschi, M. Galetti, R. A. Sinico, G. Moroni, A. Bonanni, A. Radice et al. Glomerular Autoimmune Multicomponents of Human Lupus Nephritis In Vivo (2): Planted Antigens. Journal of the American Society of Nephrology : JASN, 2015;26:1905-24.

[134] H. Nahamura, K. Yoshida, Y. Kishima, H. Enomoto, H. Uyama, T. Kuroda et al. Circulating auto-antibody against hepatoma-derived growth factor (HDGF) in patients with ulcerative colitis. Hepatogastroenterology, 2004;51:470-5.

[135] A. M. Rosenberg, D. M. Cordeiro. Relationship between sex and antibodies to high mobility group proteins 1 and 2 in juvenile idiopathic arthritis. J Rheumatol, 2000;27:2489-93.

[136] S. Barnay-Verdier, L. Fattoum, C. Borde, S. Kaveri, S. Gibot, V. Maréchal. Emergence of autoantibodies to HMGB1 is associated with survival in patients with septic shock. Intensive care medicine, 2011;37:957-62.

[137] J. N. Douglas, L. A. Gardner, H. E. Salapa, M. C. Levin. Antibodies to the RNA Binding Protein Heterogeneous Nuclear Ribonucleoprotein A1 Colocalize to Stress Granules Resulting in Altered RNA and Protein Levels in a Model of Neurodegeneration in Multiple Sclerosis. J Clin Cell Immunol, 2016;7:402.

[138] M. F. Konig, J. T. Giles, P. A. Nigrovic, F. Andrade. Antibodies to native and citrullinated RA33 (hnRNP A2/B1) challenge citrullination as the inciting principle underlying loss of tolerance in rheumatoid arthritis. Annals of the rheumatic diseases, 2016;75:2022-8.

[139] S. Siapka, M. Patrinou-Georgoula, P. G. Vlachoyiannopoulos, A. Guialis. Multiple specificities of autoantibodies against hnRNP A/B proteins in systemic rheumatic diseases and hnRNP $L$ as an associated novel autoantigen. Autoimmunity, 2007;40:223-33.

[140] N. H. Heegaard, M. R. Larsen, T. Muncrief, A. Wiik, P. Roepstorff. Heterogeneous nuclear ribonucleoproteins C1/C2 identified as autoantigens by biochemical and mass spectrometric methods. Arthritis research, 2000;2:407-14.

[141] D. Stanek, J. Vencovsky, J. Kafkova, I. Raska. Heterogenous nuclear RNP C1 and C2 core proteins are targets for an autoantibody found in the serum of a patient with systemic sclerosis and psoriatic arthritis. Arthritis and rheumatism, 1997;40:2172-7.

[142] K. Skriner, W. Hueber, E. Süleymanoglu, E. Höfler, V. Krenn, J. Smolen et al. AUF1, the regulator of tumor necrosis factor alpha messenger RNA decay, is targeted by autoantibodies of patients with systemic rheumatic diseases. Arthritis and rheumatism, 2008;58:511-20.

[143] Y. Zhang, H. Zhao, B. Liu, L. Li, L. Zhang, M. Bao et al. Low Level Antibodies Against Alpha-Tropomyosin Are Associated With Increased Risk of Coronary Heart Disease. Frontiers in pharmacology, 2020;11:195.

[144] K. Op De Beéck, L. Maes, K. Van den Bergh, R. Derua, E. Waelkens, K. Van Steen et al. Heterogeneous nuclear RNPs as targets of autoantibodies in systemic rheumatic diseases. Arthritis and rheumatism, 2012;64:213-21.

[145] Y. Kim, N. B. Caberoy, G. Alvarado, J. L. Davis, W. J. Feuer, W. Li. Identification of Hnrph3 as an autoantigen for acute anterior uveitis. Clin Immunol, 2011;138:60-6.

[146] L. Yang, M. Fujimoto, H. Murota, S. Serada, M. Fujimoto, H. Honda et al. Proteomic identification of heterogeneous nuclear ribonucleoprotein $\mathrm{K}$ as a novel cold-associated autoantigen in patients with secondary Raynaud's phenomenon. Rheumatology (Oxford, England), 2015;54:349-58.

[147] W. Hassfeld, E. K. Chan, D. A. Mathison, D. Portman, G. Dreyfuss, G. Steiner et al. Molecular definition of heterogeneous nuclear ribonucleoprotein R (hnRNP R) using autoimmune antibody: immunological relationship with hnRNP P. Nucleic acids research, 1998;26:43945.

[148] S. Britton, C. Froment, P. Frit, B. Monsarrat, B. Salles, P. Calsou. Cell nonhomologous end joining capacity controls SAF-A phosphorylation by DNA-PK in response to DNA double-strand breaks inducers. Cell Cycle, 2009;8:3717-22.

[149] L. Harlow, I. O. Rosas, B. R. Gochuico, T. R. Mikuls, P. F. Dellaripa, C. V. Oddis et al. Identification of citrullinated hsp90 isoforms as novel autoantigens in rheumatoid arthritis-associated interstitial lung disease. Arthritis and rheumatism, 2013;65:869-79. 
bioRxiv preprint doi: https://doi.org/10.1101/2021.07.30.454526; this version posted August 4, 2021. The copyright holder for this preprint (which was not certified by peer review) is the author/funder, who has granted bioRxiv a license to display the preprint in perpetuity. It is made available under aCC-BY 4.0 International license.

[150] H. Y. Qin, J. L. Mahon, M. A. Atkinson, P. Chaturvedi, E. Lee-Chan, B. Singh. Type 1 diabetes alters anti-hsp90 autoantibody isotype. Journal of autoimmunity, 2003;20:237-45.

[151] E. S. Pires, A. K. Choudhury, S. Idicula-Thomas, V. V. Khole. Anti-HSP90 autoantibodies in sera of infertile women identify a dominant, conserved epitope EP6 (380-389) of HSP90 beta protein. Reprod Biol Endocrinol, 2011;9:16.

[152] S. Suzuki, K. Utsugisawa, K. Iwasa, T. Satoh, Y. Nagane, H. Yoshikawa et al. Autoimmunity to endoplasmic reticulum chaperone GRP94 in myasthenia gravis. Journal of neuroimmunology, 2011;237:87-92.

[153] M. Chen, F. Aosai, H. S. Mun, K. Norose, H. Hata, A. Yano. Anti-HSP70 autoantibody formation by B-1 cells in Toxoplasma gondii-infected mice. Infect Immun, 2000;68:4893-9.

[154] M. Tishler, Y. Shoenfeld. Anti-heat-shock protein antibodies in rheumatic and autoimmune diseases. Semin Arthritis Rheum, 1996;26:55863.

[155] Y. Matsueda, Y. Arinuma, T. Nagai, S. Hirohata. Elevation of serum anti-glucose-regulated protein 78 antibodies in neuropsychiatric systemic lupus erythematosus. Lupus Sci Med, 2018;5:e000281.

[156] A. lannaccone, F. Giorgianni, D. D. New, T. J. Hollingsworth, A. Umfress, A. H. Alhatem et al. Circulating Autoantibodies in Age-Related Macular Degeneration Recognize Human Macular Tissue Antigens Implicated in Autophagy, Immunomodulation, and Protection from Oxidative Stress and Apoptosis. PloS one, 2015;10:e0145323.

[157] D. Papp, Z. Prohászka, J. Kocsis, G. Füst, D. Bánhegyi, D. A. Raynes et al. Development of a sensitive assay for the measurement of antibodies against heat shock protein binding protein 1 (HspBP1): increased levels of anti-HspBP1 lgG are prevalent in HIV infected subjects. Journal of medical virology, 2005;76:464-9.

[158] L. Horvath, L. Cervenak, M. Oroszlan, Z. Prohaszka, K. Uray, F. Hudecz et al. Antibodies against different epitopes of heat-shock protein 60 in children with type 1 diabetes mellitus. Immunol Lett, 2002;80:155-62.

[159] H. Fillit, S. Shibata, T. Sasaki, H. Spiera, L. D. Kerr, M. Blake. Autoantibodies to the protein core of vascular basement membrane heparan sulfate proteoglycan in systemic lupus erythematosus. Autoimmunity, 1993;14:243-9.

[160] M. Minohara. [Heat shock protein 105 in multiple sclerosis]. Nippon Rinsho, 2003;61:1317-22.

[161] T. Kobayashi, T. Yura, H. Yanagi. The increment of anti-ORP150 autoantibody in initial stages of atheroma in high-fat diet fed mice. The Journal of veterinary medical science, 2002;64:177-80.

[162] M. Tiumentseva, V. Morozova, A. Zakabunin, D. Korobko, N. Malkova, M. Filipenko et al. Use of the VH6-1 gene segment to code for antiinterleukin-18 autoantibodies in multiple sclerosis. Immunogenetics, 2016;68:237-46.

[163] H. D. Bremer, N. Landegren, R. Sjoberg, A. Hallgren, S. Renneker, E. Lattwein et al. ILF2 and ILF3 are autoantigens in canine systemic autoimmune disease. Sci Rep, 2018;8:4852.

[164] T. O. Ola, P. A. Biro, M. I. Hawa, J. Ludvigsson, M. Locatelli, M. A. Puglisi et al. Importin beta: a novel autoantigen in human autoimmunity identified by screening random peptide libraries on phage. Journal of autoimmunity, 2006;26:197-207.

[165] L. Y. Luo, I. Herrera, A. Soosaipillai, E. P. Diamandis. Identification of heat shock protein 90 and other proteins as tumour antigens by serological screening of an ovarian carcinoma expression library. British journal of cancer, 2002;87:339-43

[166] S. Presslauer, G. Hinterhuber, K. Cauza, R. Horvat, K. Rappersberger, K. Wolff et al. RasGAP-like protein IQGAP1 is expressed by human keratinocytes and recognized by autoantibodies in association with bullous skin disease. The Journal of investigative dermatology, 2003;120:365-71.

[167] H. Idborg, A. Zandian, A. S. Sandberg, B. Nilsson, K. Elvin, L. Truedsson et al. Two subgroups in systemic lupus erythematosus with features of antiphospholipid or Sjögren's syndrome differ in molecular signatures and treatment perspectives. Arthritis research \& therapy, 2019;21:62.

[168] L. Dyachenko, K. Havrysh, A. Lytovchenko, I. Dosenko, S. Antoniuk, V. Filonenko et al. Autoantibody Response to ZRF1 and KRR1 SEREX Antigens in Patients with Breast Tumors of Different Histological Types and Grades. Disease markers, 2016;2016:5128720.

[169] Y. Lu, P. Ye, S. L. Chen, E. M. Tan, E. K. Chan. Identification of kinectin as a novel Behçet's disease autoantigen. Arthritis research \& therapy, 2005;7:R1133-9.

[170] J. Inagaki, A. Kondo, L. R. Lopez, Y. Shoenfeld, E. Matsuura. Pregnancy loss and endometriosis: pathogenic role of anti-laminin-1 autoantibodies. Annals of the New York Academy of Sciences, 2005;1051:174-84.

[171] C. J. Peutz-Kootstra, K. Hansen, E. De Heer, C. K. Abrass, J. A. Bruijn. Differential expression of laminin chains and anti-laminin autoantibodies in experimental lupus nephritis. The Journal of pathology, 2000;192:404-12.

[172] R. Kain, H. Tadema, E. F. McKinney, A. Benharkou, R. Brandes, A. Peschel et al. High prevalence of autoantibodies to hLAMP-2 in antineutrophil cytoplasmic antibody-associated vasculitis. Journal of the American Society of Nephrology : JASN, 2012;23:556-66.

[173] D. Braunschweig, P. Krakowiak, P. Duncanson, R. Boyce, R. L. Hansen, P. Ashwood et al. Autism-specific maternal autoantibodies recognize critical proteins in developing brain. Translational psychiatry, 2013;3:e277.

[174] Y. Liu, J. He, X. Xie, G. Su, S. Teitz-Tennenbaum, M. S. Sabel et al. Serum autoantibody profiling using a natural glycoprotein microarray for the prognosis of early melanoma. J Proteome Res, 2010;9:6044-51.

[175] D. Lutomski, R. Joubert-Caron, C. Lefebure, J. Salama, C. Belin, D. Bladier et al. Anti-galectin-1 autoantibodies in serum of patients with neurological diseases. Clin Chim Acta, 1997;262:131-8.

[176] K. N. Konstantinov, Z. Galcheva-Gargova, M. Hoier-Madsen, A. Wiik, S. Ullman, P. Halberg et al. Autoantibodies to lamins A and C in sera of patients showing peripheral fluorescent antinuclear antibody pattern on HEP-2 cells. The Journal of investigative dermatology, 1990;95:304-8.

[177] A. von Mikecz, K. Konstantinov, D. S. Buchwald, L. Gerace, E. M. Tan. High frequency of autoantibodies to insoluble cellular antigens in patients with chronic fatigue syndrome. Arthritis and rheumatism, 1997;40:295-305.

[178] J. Brito, G. Biamonti, R. Caporali, C. Montecucco. Autoantibodies to human nuclear lamin B2 protein. Epitope specificity in different autoimmune diseases. Journal of immunology (Baltimore, Md : 1950), 1994;153:2268-77.

[179] M. Tanaka, M. Kishimura, S. Ozaki, F. Osakada, H. Hashimoto, M. Okubo et al. Cloning of novel soluble gp130 and detection of its neutralizing autoantibodies in rheumatoid arthritis. The Journal of clinical investigation, 2000;106:137-44.

[180] A. Gadoth, T. J. Kryzer, J. Fryer, A. McKeon, V. A. Lennon, S. J. Pittock. Microtubule-associated protein 1B: Novel paraneoplastic biomarker. Ann Neurol, 2017;81:266-77.

[181] G. Frampton, S. Moriya, J. D. Pearson, D. A. Isenberg, F. J. Ward, T. A. Smith et al. Identification of candidate endothelial cell autoantigens in systemic lupus erythematosus using a molecular cloning strategy: a role for ribosomal P protein P0 as an endothelial cell autoantigen. Rheumatology (Oxford, England), 2000;39:1114-20. 
bioRxiv preprint doi: https://doi.org/10.1101/2021.07.30.454526; this version posted August 4, 2021. The copyright holder for this preprint (which was not certified by peer review) is the author/funder, who has granted bioRxiv a license to display the preprint in perpetuity. It is made available under aCC-BY 4.0 International license.

[182] K. Suzuki, T. Nagao, M. Itabashi, Y. Hamano, R. Sugamata, Y. Yamazaki et al. A novel autoantibody against moesin in the serum of patients with MPO-ANCA-associated vasculitis. Nephrol Dial Transplant, 2014;29:1168-77.

[183] D. Marinou, G. Katsifis, G. Barouta, C. Liaskos, L. I. Sakkas, A. Tsakris et al. Major vault protein/lung resistance related protein: a novel biomarker for rheumatoid arthritis. Clinical and experimental rheumatology, 2020.

[184] C. A. von Muhlen, E. K. Chan, C. L. Peebles, H. Imai, K. Kiyosawa, E. M. Tan. Non-muscle myosin as target antigen for human autoantibodies in patients with hepatitis C virus-associated chronic liver diseases. Clinical and experimental immunology, 1995;100:67-74.

[185] D. A. Bledzhyants, R. M. Muratov, R. R. Movsesyan, Z. A. Podlubnaya. Autoantibodies to myosin light chains in the blood as early marker of myocardial injury after aortocoronary bypass surgery. Bull Exp Biol Med, 2007;144:241-5.

[186] B. A. Zasońska, H. Hlídková, E. Petrovský, S. Myronovskij, T. Nehrych, N. Negrych et al. Monodisperse magnetic poly(glycidyl methacrylate) microspheres for isolation of autoantibodies with affinity for the $46 \mathrm{kDa}$ form of unconventional Myo1C present in autoimmune patients. Mikrochimica acta, 2018;185:262.

[187] R. Mossabeb, S. Seiberler, I. Mittermann, R. Reininger, S. Spitzauer, S. Natter et al. Characterization of a novel isoform of alpha-nascent polypeptide-associated complex as IgE-defined autoantigen. The Journal of investigative dermatology, 2002;119:820-9.

[188] M. Beaulande, M. Kron, M. Hirakata, M. Härtlein. Human anti-asparaginyl-tRNA synthetase autoantibodies (anti-KS) increase the affinity of the enzyme for its tRNA substrate. FEBS letters, 2001;494:170-4.

[189] I. N. Batova, R. T. Richardson, E. E. Widgren, M. G. O'Rand. Analysis of the autoimmune epitopes on human testicular NASP using recombinant and synthetic peptides. Clinical and experimental immunology, 2000;121:201-9.

[190] Z. Qin, B. Lavingia, Y. Zou, P. Stastny. Antibodies against nucleolin in recipients of organ transplants. Transplantation, 2011;92:829-35.

[191] K. Cortés-Sarabia, C. Rodríguez-Nava, Y. Medina-Flores, O. Mata-Ruíz, J. E. López-Meza, M. D. Gómez-Cervantes et al. Production and characterization of a monoclonal antibody against the sialidase of Gardnerella vaginalis using a synthetic peptide in a MAP8 format. Appl Microbiol Biotechnol, 2020;104:6173-83.

[192] F. Le Naour, F. Brichory, D. E. Misek, C. Brechot, S. M. Hanash, L. Beretta. A distinct repertoire of autoantibodies in hepatocellular carcinoma identified by proteomic analysis. Molecular \& cellular proteomics : MCP, 2002;1:197-203.

[193] J. Liu, X. Xing, H. Huang, Y. Jiang, H. He, X. Xu et al. Identification of antigenic proteins associated with trichloroethylene-induced autoimmune disease by serological proteome analysis. Toxicology and applied pharmacology, 2009;240:393-400.

[194] J. R. Underwood, X. F. Csar, B. A. Veitch, M. T. Hearn. Characterization of the specificity of a naturally-occurring monoclonal anti-thymocyte autoantibody derived from an unimmunized, neonatal Balb/c mouse. Thymus, 1993;21:199-219.

[195] D. B. Ulanet, M. Torbenson, C. V. Dang, L. Casciola-Rosen, A. Rosen. Unique conformation of cancer autoantigen B23 in hepatoma: a mechanism for specificity in the autoimmune response. Proceedings of the National Academy of Sciences of the United States of America, 2003;100:12361-6.

[196] J. J. Devaux, M. Odaka, N. Yuki. Nodal proteins are target antigens in Guillain-Barré syndrome. Journal of the peripheral nervous system : JPNS, 2012;17:62-71.

[197] L. E. Andrade, E. K. Chan, C. L. Peebles, E. M. Tan. Two major autoantigen-antibody systems of the mitotic spindle apparatus. Arthritis and rheumatism, 1996;39:1643-53.

[198] R. L. Ochs, T. W. Stein, Jr., E. K. Chan, M. Ruutu, E. M. Tan. cDNA cloning and characterization of a novel nucleolar protein. Molecular biology of the cell, 1996;7:1015-24.

[199] S. Nagayama, T. Yokoi, H. Tanaka, Y. Kawaguchi, T. Shirasaka, T. Kamataki. Occurrence of autoantibody to protein disulfide isomerase in patients with hepatic disorder. J Toxicol Sci, 1994;19:163-9.

[200] A. Becker, N. Ludwig, A. Keller, B. Tackenberg, C. Eienbroker, W. H. Oertel et al. Myasthenia gravis: analysis of serum autoantibody reactivities to 1827 potential human autoantigens by protein macroarrays. PloS one, 2013;8:e58095.

[201] A. K. Houng, L. Maggini, C. Y. Clement, G. L. Reed. Identification and structure of activated-platelet protein-1, a protein with RNA-binding domain motifs that is expressed by activated platelets. European journal of biochemistry, 1997;243:209-18.

[202] T. C. Hsu, G. J. Tsay, T. Y. Chen, Y. C. Liu, B. S. Tzang. Anti-PCNA autoantibodies preferentially recognize C-terminal of PCNA in patients with chronic hepatitis B virus infection. Clinical and experimental immunology, 2006;144:110-6.

[203] C. Caorsi, E. Niccolai, M. Capello, R. Vallone, M. S. Chattaragada, B. Alushi et al. Protein disulfide isomerase A3-specific Th1 effector cells infiltrate colon cancer tissue of patients with circulating anti-protein disulfide isomerase A3 autoantibodies. Translational research : the journal of laboratory and clinical medicine, 2016;171:17-28.e1-2.

[204] J. Gut, U. Christen, N. Frey, V. Koch, D. Stoffler. Molecular mimicry in halothane hepatitis: biochemical and structural characterization of lipoylated autoantigens. Toxicology, 1995;97:199-224.

[205] S. E. Stanton, E. Gad, E. Ramos, L. Corulli, J. Annis, J. Childs et al. Tumor-associated autoantibodies from mouse breast cancer models are found in serum of breast cancer patients. NPJ Breast Cancer, 2021;7:50.

[206] D. C. Chang, P. Piaggi, R. L. Hanson, W. C. Knowler, C. Bogardus, J. Krakoff. Autoantibodies against PFDN2 are associated with an increased risk of type 2 diabetes: A case-control study. Diabetes Metab Res Rev, 2017;33.

[207] C. Chen, D. Liao, J. Wang, Z. Liang, Q. Yao. Anti-human protein S antibody induces tissue factor expression through a direct interaction with platelet phosphofructokinase. Thrombosis research, 2014;133:222-8.

[208] P. J. Orchard, D. R. Nascene, A. Gupta, M. E. Taisto, L. Higgins, T. W. Markowski et al. Cerebral adrenoleukodystrophy is associated with loss of tolerance to profilin. Eur J Immunol, 2019;49:947-53.

[209] A. Kimura, T. Sakurai, A. Koumura, M. Yamada, Y. Hayashi, Y. Tanaka et al. High prevalence of autoantibodies against phosphoglycerate mutase 1 in patients with autoimmune central nervous system diseases. Journal of neuroimmunology, 2010;219:105-8.

[210] D. Xiang, Q. Xia, D. Chen, X. Feng, Y. Zhao, Y. Liu et al. Detection of D-3-phosphoglycerate dehydrogenase autoantibodies in patients with autoimmune hepatitis: Clinical significance evaluation. Hepatology research : the official journal of the Japan Society of Hepatology, 2011;41:867-76.

[211] M. Wieczorek, A. Czernik. Paraneoplastic pemphigus: a short review. Clin Cosmet Investig Dermatol, 2016;9:291-5.

[212] K. Ueda, T. Nakanishi, A. Shimizu, T. Takubo, N. Matsuura. Identification of L-plastin autoantibody in plasma of patients with non-Hodgkin's lymphoma using a proteomics-based analysis. Ann Clin Biochem, 2008;45:65-9.

[213] C. A. Mecoli, B. L. Adler, Q. Yang, L. K. Hummers, A. Rosen, L. Casciola-Rosen et al. Cancer in Systemic Sclerosis: Analysis of Antibodies Against Components of the Th/To Complex. Arthritis \& rheumatology (Hoboken, NJ), 2020.

[214] A. Loshaj-Shala, M. Colzani, K. Brezovska, A. Poceva Panovska, L. Suturkova, G. Beretta. Immunoproteomic identification of antigenic candidate Campylobacter jejuni and human peripheral nerve proteins involved in Guillain-Barré syndrome. Journal of neuroimmunology, 2018;317:77-83. 
bioRxiv preprint doi: https://doi.org/10.1101/2021.07.30.454526; this version posted August 4, 2021. The copyright holder for this preprint (which was not certified by peer review) is the author/funder, who has granted bioRxiv a license to display the preprint in perpetuity. It is made available under aCC-BY 4.0 International license.

[215] A. Kratz, M. W. Harding, J. Craft, C. G. Mackworth-Young, R. E. Handschumacher. Autoantibodies against cyclophilin in systemic lupus erythematosus and Lyme disease. Clinical and experimental immunology, 1992;90:422-7.

[216] R. Karasawa, S. Ozaki, K. Nishioka, T. Kato. Autoantibodies to peroxiredoxin I and IV in patients with systemic autoimmune diseases. Microbiology and immunology, 2005;49:57-65.

[217] L. H. Lin, Y. W. Xu, L. S. Huang, C. Q. Hong, T. T. Zhai, L. D. Liao et al. Serum proteomic-based analysis identifying autoantibodies against PRDX2 and PRDX3 as potential diagnostic biomarkers in nasopharyngeal carcinoma. Clinical proteomics, 2017;14:6.

[218] S. Kobayashi, T. Hiwasa, T. Arasawa, A. Kagaya, S. Ishii, H. Shimada et al. Identification of specific and common diagnostic antibody markers for gastrointestinal cancers by SEREX screening using testis cDNA phage library. Oncotarget, 2018;9:18559-69.

[219] C. Schild-Poulter, A. Su, A. Shih, O. P. Kelly, M. J. Fritzler, R. Goldstein et al. Association of autoantibodies with Ku and DNA repair proteins in connective tissue diseases. Rheumatology (Oxford, England), 2008;47:165-71.

[220] K. H. Sun, S. J. Tang, Y. S. Wang, W. J. Lin, R. I. You. Autoantibodies to dsDNA cross-react with the arginine-glycine-rich domain of heterogeneous nuclear ribonucleoprotein A2 (hnRNP A2) and promote methylation of hnRNP A2. Rheumatology (Oxford), 2003;42:154-61.

[221] E. Feist, U. Kuckelkorn, T. Dörner, H. Dönitz, S. Scheffler, F. Hiepe et al. Autoantibodies in primary Sjögren's syndrome are directed against proteasomal subunits of the alpha and beta type. Arthritis and rheumatism, 1999;42:697-702.

[222] C. Bohring, W. Krause. Characterization of spermatozoa surface antigens by antisperm antibodies and its influence on acrosomal exocytosis. Am J Reprod Immunol, 2003;50:411-9.

[223] K. Sugimoto, T. Hiwasa, K. Shibuya, S. Hirano, M. Beppu, S. Isose et al. Novel autoantibodies against the proteasome subunit PSMA7 in amyotrophic lateral sclerosis. Journal of neuroimmunology, 2018;325:54-60.

[224] S. Scheffler, U. Kuckelkorn, K. Egerer, T. Dörner, K. Reiter, A. Soza et al. Autoimmune reactivity against the 20S-proteasome includes immunosubunits LMP2 (beta1i), MECL1 (beta2i) and LMP7 (beta5i). Rheumatology (Oxford, England), 2008;47:622-6.

[225] Z. Mojtahedi, A. Safaei, Z. Yousefi, A. Ghaderi. Immunoproteomics of HER2-positive and HER2-negative breast cancer patients with positive lymph nodes. Omics : a journal of integrative biology, 2011;15:409-18.

[226] M. Roessler, W. Rollinger, L. Mantovani-Endl, M. L. Hagmann, S. Palme, P. Berndt et al. Identification of PSME3 as a novel serum tumor marker for colorectal cancer by combining two-dimensional polyacrylamide gel electrophoresis with a strictly mass spectrometry-based approach for data analysis. Mol Cell Proteomics, 2006;5:2092-101.

[227] C. Montecucco, R. Caporali, F. Cobianchi, G. Biamonti. Identification of autoantibodies to the I protein of the heterogeneous nuclear ribonucleoprotein complex in patients with systemic sclerosis. Arthritis and rheumatism, 1996;39:1669-76.

[228] P. G. Vlachoyiannopoulos, S. Frillingos, A. G. Tzioufas, K. Seferiadis, H. M. Moutsopoulos, O. Tsolas. Circulating antibodies to prothymosin alpha in systemic lupus erythematosus. Clinical immunology and immunopathology, 1989;53:151-60.

[229] D. F. Fiorentino, M. Presby, A. N. Baer, M. Petri, K. E. Rieger, M. Soloski et al. PUF60: a prominent new target of the autoimmune response in dermatomyositis and Sjögren's syndrome. Annals of the rheumatic diseases, 2016;75:1145-51.

[230] I. Schepens, F. Jaunin, N. Begre, U. Läderach, K. Marcus, T. Hashimoto et al. The protease inhibitor alpha-2-macroglobulin-like-1 is the p170 antigen recognized by paraneoplastic pemphigus autoantibodies in human. PloS one, 2010;5:e12250.

[231] S. Thébault, D. Gilbert, M. Hubert, L. Drouot, N. Machour, C. Lange et al. Orderly pattern of development of the autoantibody response in (New Zealand White $x$ BXSB)F1 lupus mice: characterization of target antigens and antigen spreading by two-dimensional gel electrophoresis and mass spectrometry. Journal of immunology (Baltimore, Md : 1950), 2002;169:4046-53.

[232] J. H. Vaughan, J. R. Valbracht, M. D. Nguyen, H. H. Handley, R. S. Smith, K. Patrick et al. Epstein-Barr virus-induced autoimmune responses. I. Immunoglobulin $\mathrm{M}$ autoantibodies to proteins mimicking and not mimicking Epstein-Barr virus nuclear antigen-1. The Journal of clinical investigation, 1995;95:1306-15.

[233] K. Doe, K. Nozawa, K. Hiruma, Y. Yamada, Y. Matsuki, S. Nakano et al. Antibody against chromatin assembly factor-1 is a novel autoantibody specifically recognized in systemic lupus erythematosus. Lupus, 2014;23:1031-41.

[234] M. Wagatsuma, M. Kimura, R. Suzuki, F. Takeuchi, K. Matsuta, H. Watanabe. Ezrin, radixin and moesin are possible auto-immune antigens in rheumatoid arthritis. Molecular immunology, 1996;33:1171-6.

[235] R. H. Scofield, J. B. Harley. Autoantigenicity of Ro/SSA antigen is related to a nucleocapsid protein of vesicular stomatitis virus. Proceedings of the National Academy of Sciences of the United States of America, 1991;88:3343-7.

[236] Y. Yamasaki, S. Narain, L. Hernandez, T. Barker, K. Ikeda, M. S. Segal et al. Autoantibodies against the replication protein A complex in systemic lupus erythematosus and other autoimmune diseases. Arthritis research \& therapy, 2006;8:R111.

[237] T. Sato, T. Uchiumi, R. Kominami, M. Arakawa. Autoantibodies specific for the 20-KDal ribosomal large subunit protein L12. Biochemical and biophysical research communications, 1990;172:496-502.

[238] M. Absi, J. P. La Vergne, A. Marzouki, F. Giraud, D. Rigal, A. M. Reboud et al. Heterogeneity of ribosomal autoantibodies from human, murine and canine connective tissue diseases. Immunology letters, 1989;23:35-41.

[239] A. Guialis, M. Patrinou-Georgoula, N. Tsifetaki, V. Aidinis, C. E. Sekeris, H. M. Moutsopoulos. Anti-5S RNA/protein (RNP) antibody levels correlate with disease activity in a patient with systemic lupus erythematosus (SLE) nephritis. Clinical and experimental immunology, 1994;95:385-9.

[240] K. Elkon, E. Bonfa, R. Llovet, W. Danho, H. Weissbach, N. Brot. Properties of the ribosomal P2 protein autoantigen are similar to those of foreign protein antigens. Proceedings of the National Academy of Sciences of the United States of America, 1988;85:5186-9.

[241] K. Elkon, H. Weissbach, N. Brot. Central nervous system function in systemic lupus erythematosus. Neurochemical research, 1990;15:4016.

[242] K. T. Tycowski, M. D. Shu, J. A. Steitz. A small nucleolar RNA is processed from an intron of the human gene encoding ribosomal protein S3. Genes \& development, 1993;7:1176-90.

[243] J. Qiu, G. Choi, L. Li, H. Wang, S. J. Pitteri, S. R. Pereira-Faca et al. Occurrence of autoantibodies to annexin I, 14-3-3 theta and LAMR1 in prediagnostic lung cancer sera. Journal of clinical oncology : official journal of the American Society of Clinical Oncology, 2008;26:5060-6.

[244] J. M. Yang, B. Hildebrandt, C. Luderschmidt, K. M. Pollard. Human scleroderma sera contain autoantibodies to protein components specific to the U3 small nucleolar RNP complex. Arthritis and rheumatism, 2003;48:210-7.

[245] K. Kaji, N. Fertig, T. A. Medsger, Jr., T. Satoh, K. Hoshino, Y. Hamaguchi et al. Autoantibodies to RuvBL1 and RuvBL2: a novel systemic sclerosis-related antibody associated with diffuse cutaneous and skeletal muscle involvement. Arthritis care \& research, 2014;66:575-84.

[246] J. D. Pauling, G. Salazar, H. Lu, Z. E. Betteridge, S. Assassi, M. D. Mayes et al. Presence of anti-eukaryotic initiation factor-2B, antiRuvBL1/2 and anti-synthetase antibodies in patients with anti-nuclear antibody negative systemic sclerosis. Rheumatology (Oxford, England), 2018;57:712-7. 
bioRxiv preprint doi: https://doi.org/10.1101/2021.07.30.454526; this version posted August 4, 2021. The copyright holder for this preprint (which was not certified by peer review) is the author/funder, who has granted bioRxiv a license to display the preprint in perpetuity. It is made available under aCC-BY 4.0 International license.

[247] A. M. Abreu-Velez, M. S. Howard, K. Hashimoto, T. Hashimoto. Autoantibodies to sweat glands detected by different methods in serum and in tissue from patients affected by a new variant of endemic pemphigus foliaceus. Archives of dermatological research, 2009;301:711-8.

[248] P. Margutti, M. Sorice, F. Conti, F. Delunardo, M. Racaniello, C. Alessandri et al. Screening of an endothelial cDNA library identifies the Cterminal region of Nedd5 as a novel autoantigen in systemic lupus erythematosus with psychiatric manifestations. Arthritis research \& therapy, 2005;7:R896-903.

[249] D. Lefranc, D. Launay, S. Dubucquoi, J. de Seze, P. Dussart, M. Vermersch et al. Characterization of discriminant human brain antigenic targets in neuropsychiatric systemic lupus erythematosus using an immunoproteomic approach. Arthritis and rheumatism, 2007;56:342032.

[250] R. L. Bates, S. J. Payne, S. L. Drury, P. N. Nelson, D. A. Isenberg, J. J. Murphy et al. The prevalence and clinical significance of autoantibodies to plasminogen activator inhibitor 1 in systemic lupus erythematosus. Lupus, 2003;12:617-22.

[251] S. Yokota, H. Kubota, Y. Matsuoka, M. Naitoh, D. Hirata, S. Minota et al. Prevalence of HSP47 antigen and autoantibodies to HSP47 in the sera of patients with mixed connective tissue disease. Biochemical and biophysical research communications, 2003;303:413-8.

[252] Z. Chai, B. Sarcevic, A. Mawson, B. H. Toh. SET-related cell division autoantigen-1 (CDA1) arrests cell growth. J Biol Chem, 2001;276:33665-74.

[253] H. M. Hwang, C. K. Heo, H. J. Lee, S. S. Kwak, W. H. Lim, J. S. Yoo et al. Identification of anti-SF3B1 autoantibody as a diagnostic marker in patients with hepatocellular carcinoma. Journal of translational medicine, 2018;16:177.

[254] A. Kistner, M. B. Bigler, K. Glatz, S. B. Egli, F. S. Baldin, F. A. Marquardsen et al. Characteristics of autoantibodies targeting 14-3-3 proteins and their association with clinical features in newly diagnosed giant cell arteritis. Rheumatology (Oxford, England), 2017;56:829-34.

[255] Y. Hosono, R. Nakashima, S. Serada, K. Murakami, Y. Imura, H. Yoshifuji et al. Splicing factor proline/glutamine-rich is a novel autoantigen of dermatomyositis and associated with anti-melanoma differentiation-associated gene 5 antibody. Journal of autoimmunity, 2017;77:11622.

[256] B. F. Chumpitazi, L. Bouillet, M. T. Drouet, L. Kuhn, J. Garin, J. P. Zarski et al. Biological autoimmunity screening in hepatitis C patients by anti-HepG2 lysate and anti-heat shock protein 70.1 autoantibodies. European journal of clinical microbiology \& infectious diseases : official publication of the European Society of Clinical Microbiology, 2009;28:137-46.

[257] M. Kubo, H. Ihn, M. Kuwana, Y. Asano, T. Tamaki, K. Yamane et al. Anti-U5 snRNP antibody as a possible serological marker for scleroderma-polymyositis overlap. Rheumatology (Oxford, England), 2002;41:531-4.

[258] D. Hof, K. Cheung, D. J. de Rooij, F. H. van den Hoogen, G. J. Pruijn, W. J. van Venrooij et al. Autoantibodies specific for apoptotic U1-70K are superior serological markers for mixed connective tissue disease. Arthritis Res Ther, 2005;7:R302-9.

[259] A. M. Yamamoto, Z. Amoura, C. Johannet, A. L. Jeronimo, H. Campos, S. Koutouzov et al. Quantitative radioligand assays using de novosynthesized recombinant autoantigens in connective tissue diseases: new tools to approach the pathogenic significance of anti-RNP antibodies in rheumatic diseases. Arthritis and rheumatism, 2000;43:689-98.

[260] T. Mimori, M. Hinterberger, I. Pettersson, J. A. Steitz. Autoantibodies to the U2 small nuclear ribonucleoprotein in a patient with sclerodermapolymyositis overlap syndrome. The Journal of biological chemistry, 1984;259:560-5.

[261] J. D. Huntriss, D. S. Latchman, D. G. Williams. Lupus autoantibodies discriminate between the highly homologous Sm polypeptides B/B and $\mathrm{SmN}$ by binding an epitope restricted to B/B'. Clinical and experimental immunology, 1993;92:263-7.

[262] H. Brahms, J. Raymackers, A. Union, F. de Keyser, L. Meheus, R. Luhrmann. The C-terminal RG dipeptide repeats of the spliceosomal Sm proteins D1 and D3 contain symmetrical dimethylarginines, which form a major B-cell epitope for anti-Sm autoantibodies. The Journal of biological chemistry, 2000;275:17122-9.

[263] M. T. McClain, P. A. Ramsland, K. M. Kaufman, J. A. James. Anti-sm autoantibodies in systemic lupus target highly basic surface structures of complexed spliceosomal autoantigens. Journal of immunology (Baltimore, Md : 1950), 2002;168:2054-62.

[264] M. Satoh, J. Y. Chan, S. J. Ross, A. Ceribelli, I. Cavazzana, F. Franceschini et al. Autoantibodies to survival of motor neuron complex in patients with polymyositis: immunoprecipitation of D, E, F, and G proteins without other components of small nuclear ribonucleoproteins. Arthritis and rheumatism, 2011;63:1972-8.

[265] M. van Blitterswijk, S. Gulati, E. Smoot, M. Jaffa, N. Maher, B. T. Hyman et al. Anti-superoxide dismutase antibodies are associated with survival in patients with sporadic amyotrophic lateral sclerosis. Amyotrophic lateral sclerosis : official publication of the World Federation of Neurology Research Group on Motor Neuron Diseases, 2011;12:430-8.

[266] M. Garbarz, D. Dhermy, O. Bournier, A. Bezeaud, P. Boivin. Anti-spectrin in sera containing smooth muscle autoantibodies from patients with chronic active hepatitis. Clinical and experimental immunology, 1981;43:87-93.

[267] A. Zaninoni, C. Vercellati, F. G. Imperiali, A. P. Marcello, B. Fattizzo, E. Fermo et al. Detection of red blood cell antibodies in mitogenstimulated cultures from patients with hereditary spherocytosis. Transfusion, 2015;55:2930-8.

[268] P. J. Utz, M. Hottelet, T. M. Le, S. J. Kim, M. E. Geiger, W. J. van Venrooij et al. The 72-kDa component of signal recognition particle is cleaved during apoptosis. The Journal of biological chemistry, 1998;273:35362-70.

[269] H. Imai, E. K. Chan, K. Kiyosawa, X. D. Fu, E. M. Tan. Novel nuclear autoantigen with splicing factor motifs identified with antibody from hepatocellular carcinoma. The Journal of clinical investigation, 1993;92:2419-26.

[270] N. lizuka, K. Okamoto, R. Matsushita, M. Kimura, K. Nagai, M. Arito et al. Identification of autoantigens specific for systemic lupus erythematosus with central nervous system involvement. Lupus, 2010;19:717-26.

[271] K. Overzet, T. J. Gensler, S. J. Kim, M. E. Geiger, W. J. van Venrooij, K. M. Pollard et al. Small nucleolar RNP scleroderma autoantigens associate with phosphorylated serine/arginine splicing factors during apoptosis. Arthritis and rheumatism, 2000;43:1327-36.

[272] P. Santoro, M. De Andrea, G. Migliaretti, C. Trapani, S. Landolfo, M. Gariglio. High prevalence of autoantibodies against the nuclear high mobility group (HMG) protein SSRP1 in sera from patients with systemic lupus erythematosus, but not other rheumatic diseases. The Journal of rheumatology, 2002;29:90-3.

[273] A. Cortini, S. Bembich, L. Marson, E. Cocco, P. Edomi. Identification of novel non-myelin biomarkers in multiple sclerosis using an improved phage-display approach. PloS one, 2019;14:e0226162.

[274] Z. Betteridge, H. Gunawardena, J. North, J. Slinn, N. McHugh. Identification of a novel autoantibody directed against small ubiquitin-like modifier activating enzyme in dermatomyositis. Arthritis and rheumatism, 2007;56:3132-7.

[275] B. Niland, G. Miklossy, K. Banki, W. E. Biddison, L. Casciola-Rosen, A. Rosen et al. Cleavage of transaldolase by granzyme B causes the loss of enzymatic activity with retention of antigenicity for multiple sclerosis patients. Journal of immunology (Baltimore, Md : 1950), 2010;184:4025-32. 
bioRxiv preprint doi: https://doi.org/10.1101/2021.07.30.454526; this version posted August 4, 2021. The copyright holder for this preprint (which was not certified by peer review) is the author/funder, who has granted bioRxiv a license to display the preprint in perpetuity. It is made available under aCC-BY 4.0 International license.

[276] Y. J. Lee, W. H. Ting, Y. W. Yang, C. J. Lin, Y. T. Hsieh, C. Y. Huang et al. HLA-DQ genotype and biochemical characterization of antitransglutaminase 2 antibodies in patients with type 1 diabetes mellitus in Taiwan. FASEB journal : official publication of the Federation of American Societies for Experimental Biology, 2020;34:8459-74.

[277] Y. Meng, M. Zhang, X. Zhao, Y. Cheng, R. Jia, Y. Wang et al. Decreased serum thrombospondin-1 and elevation of its autoantibody are associated with multiple exacerbated clinical manifestations in systemic lupus erythematosus. Clinical rheumatology, 2018;37:2707-14.

[278] M. Muto, M. Mori, T. Hiwasa, M. Takiguchi, Y. Iwadate, A. Uzawa et al. Novel serum autoantibodies against talin1 in multiple sclerosis: Possible pathogenetic roles of the antibodies. Journal of neuroimmunology, 2015;284:30-6.

[279] A. Schwenzer, X. Jiang, T. R. Mikuls, J. B. Payne, H. R. Sayles, A. M. Quirke et al. Identification of an immunodominant peptide from citrullinated tenascin-C as a major target for autoantibodies in rheumatoid arthritis. Annals of the rheumatic diseases, 2016;75:1876-83.

[280] K. Mariampillai, B. Granger, D. Amelin, M. Guiguet, E. Hachulla, F. Maurier et al. Development of a New Classification System for Idiopathic Inflammatory Myopathies Based on Clinical Manifestations and Myositis-Specific Autoantibodies. JAMA neurology, 2018;75:1528-37.

[281] X. Geng, L. Biancone, H. H. Dai, J. J. Lin, N. Yoshizaki, A. Dasgupta et al. Tropomyosin isoforms in intestinal mucosa: production of autoantibodies to tropomyosin isoforms in ulcerative colitis. Gastroenterology, 1998;114:912-22.

[282] R. Gajbhiye, A. Sonawani, S. Khan, A. Suryawanshi, S. Kadam, N. Warty et al. Identification and validation of novel serum markers for early diagnosis of endometriosis. Hum Reprod, 2012;27:408-17.

[283] A. Kimura, T. Sakurai, M. Yamada, A. Koumura, Y. Hayashi, Y. Tanaka et al. Anti-endothelial cell antibodies in patients with cerebral small vessel disease. Curr Neurovasc Res, 2012;9:296-301.

[284] P. Enarson, J. B. Rattner, Y. Ou, K. Miyachi, T. Horigome, M. J. Fritzler. Autoantigens of the nuclear pore complex. J Mol Med (Berl), 2004;82:423-33.

[285] K. Lorenz, S. Beck, M. M. Keilani, J. Wasielica-Poslednik, N. Pfeiffer, F. H. Grus. Course of serum autoantibodies in patients after acute angle-closure glaucoma attack. Clinical \& experimental ophthalmology, 2017;45:280-7.

[286] X. Zhao, Y. Cheng, Y. Gan, R. Jia, L. Zhu, X. Sun. Anti-tubulin-alpha-1C autoantibody in systemic lupus erythematosus: a novel indicator of disease activity and vasculitis manifestations. Clinical rheumatology, 2018;37:1229-37.

[287] T. Matthes, A. Wolff, P. Soubiran, F. Gros, G. Dighiero. Antitubulin antibodies. II. Natural autoantibodies and induced antibodies recognize different epitopes on the tubulin molecule. Journal of immunology (Baltimore, Md : 1950), 1988;141:3135-41.

[288] A. Kimura, N. Yoshikura, A. Koumura, Y. Hayashi, Y. Kobayashi, I. Kobayashi et al. Identification of target antigens of naturally occurring autoantibodies in cerebrospinal fluid. Journal of proteomics, 2015;128:450-7.

[289] L. Prasannan, D. E. Misek, R. Hinderer, J. Michon, J. D. Geiger, S. M. Hanash. Identification of beta-tubulin isoforms as tumor antigens in neuroblastoma. Clinical cancer research : an official journal of the American Association for Cancer Research, 2000;6:3949-56.

[290] E. B. Stubbs, Jr., M. A. Fisher, G. J. Siegel. Anti-tubulin antibodies in a sensorimotor neuropathy patient alter tubulin polymerization. Acta neuropathologica, 1998;95:302-5.

[291] Y. Muro, Y. Ogawa, Y. Kato, M. Hagiwara. Autoantibody to thioredoxin reductase in an ovarian cancer patient. Biochemical and biophysical research communications, 1998;242:267-71.

[292] Z. E. Betteridge, H. Gunawardena, H. Chinoy, J. North, W. E. Ollier, R. G. Cooper et al. Clinical and human leucocyte antigen class II haplotype associations of autoantibodies to small ubiquitin-like modifier enzyme, a dermatomyositis-specific autoantigen target, in UK Caucasian adult-onset myositis. Annals of the rheumatic diseases, 2009;68:1621-5.

[293] A. F. Pluta, W. C. Earnshaw. Specific interaction between human kinetochore protein CENP-C and a nucleolar transcriptional regulator. The Journal of biological chemistry, 1996;271:18767-74.

[294] X. Li, J. Sun, R. Mu, Y. Gan, G. Wang, J. He et al. The clinical significance of ubiquitin carboxyl hydrolase L1 and its autoantibody in neuropsychiatric systemic lupus erythematosus. Clinical and experimental rheumatology, 2019;37:474-80.

[295] Y. Zhou, J. Cui, H. Du. Autoantibody-targeted TAAs in pancreatic cancer: A comprehensive analysis. Pancreatology, 2019;19:760-8.

[296] K. Miyachi, H. Hosaka, N. Nakamura, H. Miyakawa, T. Mimori, M. Shibata et al. Anti-p97/VCP antibodies: an autoantibody marker for a subset of primary biliary cirrhosis patients with milder disease? Scand J Immunol, 2006;63:376-82.

[297] F. J. Li, R. Surolia, H. Li, Z. Wang, T. Kulkarni, G. Liu et al. Autoimmunity to Vimentin Is Associated with Outcomes of Patients with Idiopathic Pulmonary Fibrosis. Journal of immunology (Baltimore, Md : 1950), 2017;199:1596-605.

[298] J. Mao, J. Ladd, E. Gad, L. Rastetter, M. M. Johnson, E. Marzbani et al. Mining the pre-diagnostic antibody repertoire of TgMMTV-neu mice to identify autoantibodies useful for the early detection of human breast cancer. Journal of translational medicine, 2014;12:121.

[299] E. L. Paley, N. Alexandrova, L. Smelansky. Tryptophanyl-tRNA synthetase as a human autoantigen. Immunology letters, 1995;48:201-7.

[300] S. Hoa, M. Hudson, Y. Troyanov, S. Proudman, J. Walker, W. Stevens et al. Single-specificity anti-Ku antibodies in an international cohort of 2140 systemic sclerosis subjects: clinical associations. Medicine (Baltimore), 2016;95:e4713.

[301] M. Mahler, F. W. Miller, M. J. Fritzler. Idiopathic inflammatory myopathies and the anti-synthetase syndrome: a comprehensive review. Autoimmunity reviews, 2014;13:367-71.

[302] R. Morgenroth, C. Reichardt, J. Steffen, S. Busse, R. Frank, H. Heidecke et al. Autoantibody Formation and Mapping of Immunogenic Epitopes against Cold-Shock-Protein YB-1 in Cancer Patients and Healthy Controls. Cancers, 2020;12.

[303] D. Braunschweig, P. Krakowiak, P. Duncanson, R. Boyce, R. L. Hansen, P. Ashwood et al. Autism-specific maternal autoantibodies recognize critical proteins in developing brain. Translational psychiatry, 2013;3:e277.

[304] M. H. van Beers-Tas, A. Marotta, M. Boers, W. P. Maksymowych, D. van Schaardenburg. A prospective cohort study of 14-3-3eta in ACPA and/or RF-positive patients with arthralgia. Arthritis research \& therapy, 2016;18:76.

[305] R. Chakravarti, K. Gupta, M. Swain, B. Willard, J. Scholtz, L. G. Svensson et al. 14-3-3 in Thoracic Aortic Aneurysms: Identification of a Novel Autoantigen in Large Vessel Vasculitis. Arthritis \& rheumatology (Hoboken, NJ), 2015;67:1913-21.

[306] X. M. Teitsma, J. Devenport, J. W. G. Jacobs, A. Petho-Schramm, M. E. A. Borm, P. Budde et al. Comprehensive exploratory autoantibody profiling in patients with early rheumatoid arthritis treated with methotrexate or tocilizumab. PloS one, 2020;15:e0241189. 

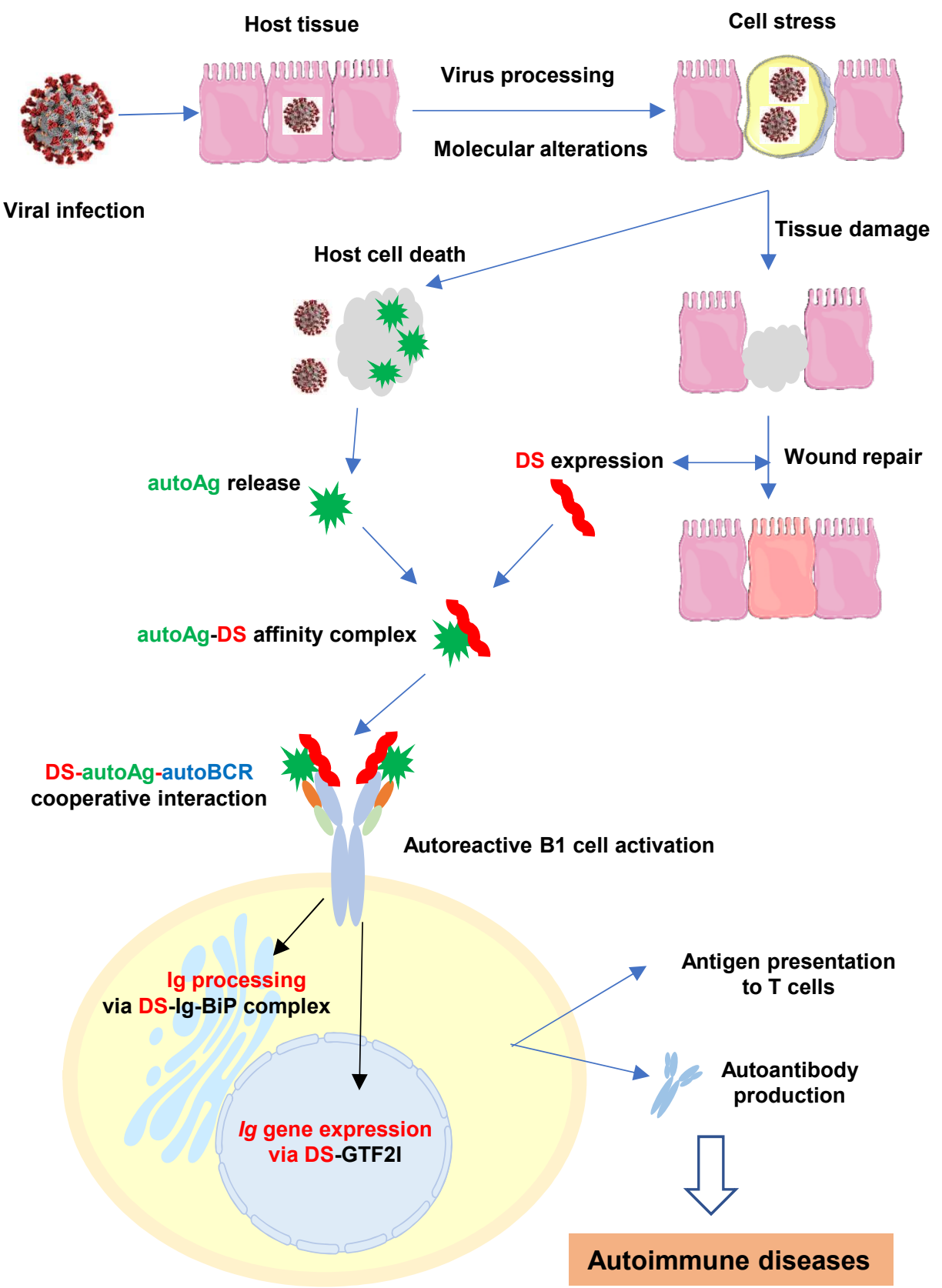

Fig. 1. A model on how viral infections lead to autoimmune diseases. Viral infections induce extensive host molecular changes, cell death, and tissue damage. AutoAgs shed from apototic cells form affinity complexes with DS that is overexpressed in the wound area. Cooperative binding of DS-autoAg complexes to autoBCRs activate autoreactive B1 cells. Once internalized via autoBCR, DS engages Ig-processing complexes in the ER and GTF2I in the nucleus to facilitate Ig production. Activated B1 cells secrete autoantibodies and may also present autoAgs to autoreactive T cells, which then leads to autoimmune diseases. 
bioRxiv preprint doi: https://doi.org/10 1101/2021.07 30.454526; this version posted August 4, 2021. The copyright holder for this preprint (which was not certified by peer review) is the author/funder, who has granted bioRxiv a license to display the preprint in perpetuity. It is made available under aCC-BY 4.0 International license.

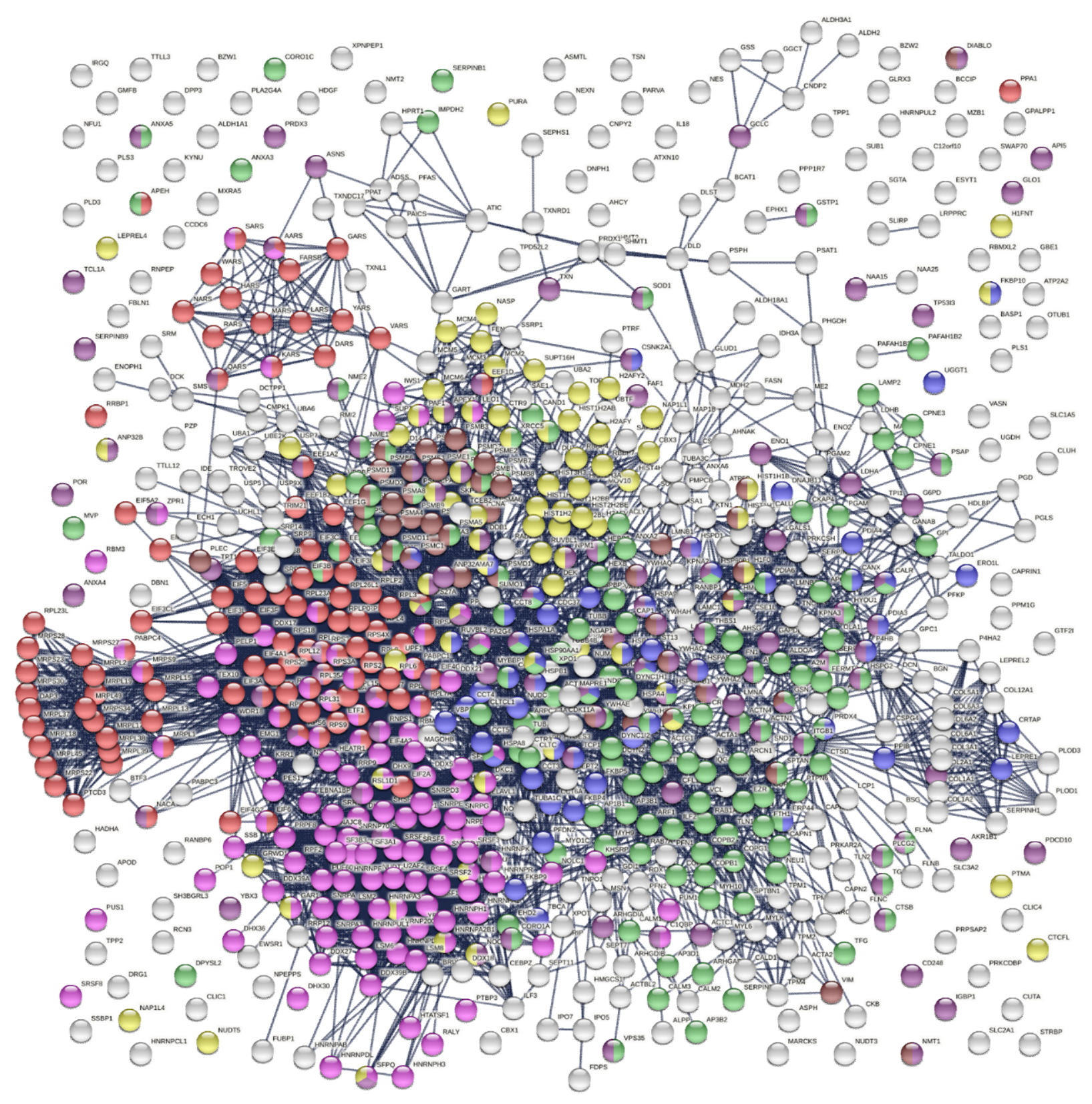

Fig. 2. The master autoAg-ome of 751 DS-affinity proteins identified from 6 cell types forms a highly interacting connected network. Lines represent protein-protein interactions with the highest confidence cutoff. Colored proteins are associated with translation (104 proteins, red), RNA processing (120 proteins, pink), protein folding (53 proteins, blue), vesicle-mediated transport (141 proteins, green), chromosome organization (76 proteins, yellow), regulation of cell death (110 proteins, dark purple), and apoptosis (46 proteins, brown). 
bioRxiv preprint doi: https://doi.org/10.1101/2021.07.30.454526; this version posted August 4, 2021. The copyright holder for this preprint (which was not certified by peer review) is the author/funder, who has granted bioRxiv a license to display the preprint in perpetuity. It is made available under aCC-BY 4.0 International license.

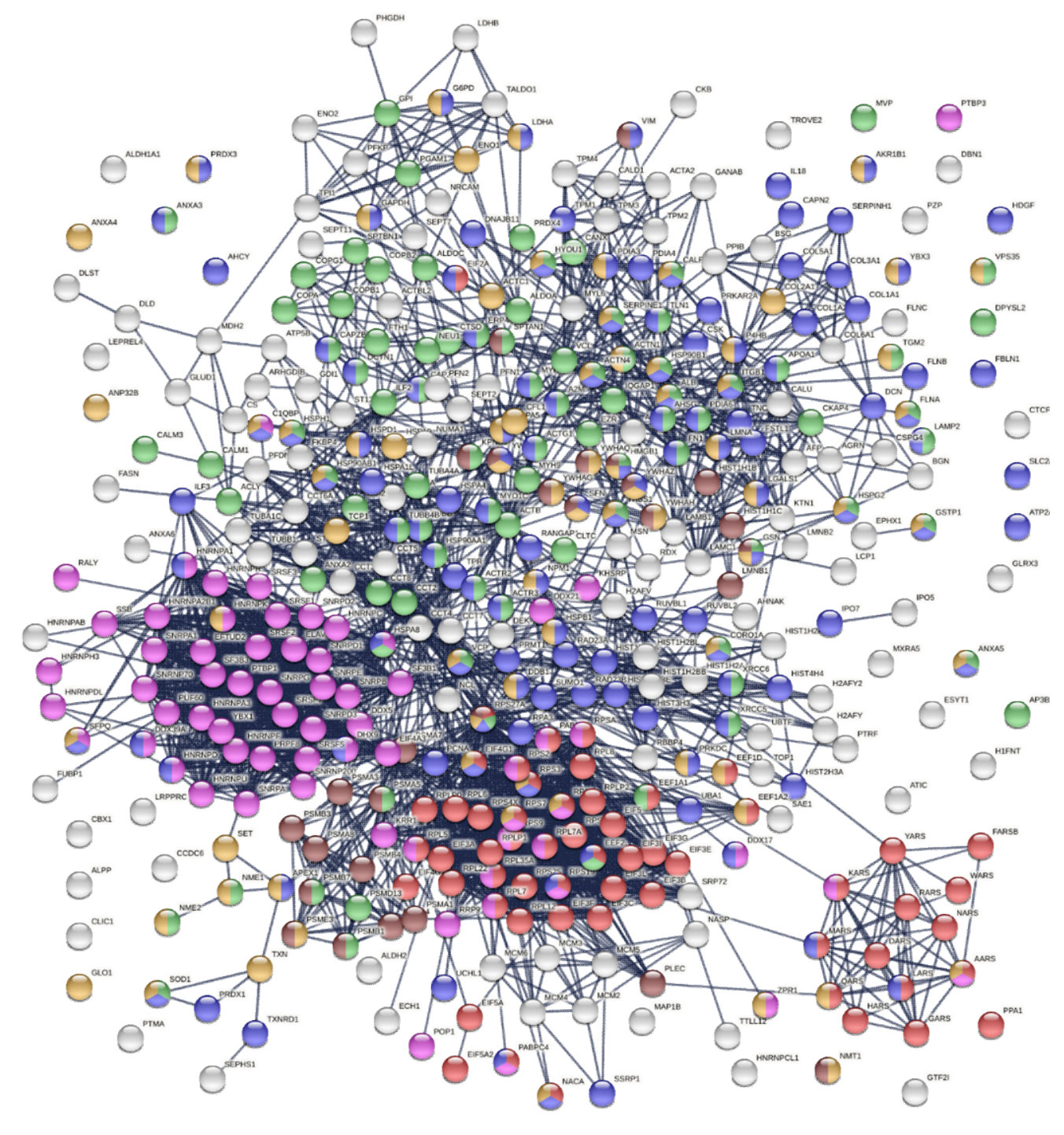

Fig. 3. Protein interaction network of the 400 confirmed autoAgs. Lines represent protein-protein interactions with highest confidence. Colored proteins are associated with translation (57 proteins, red), RNA processing (65 proteins, pink), vesicle-mediated transport (89 proteins, green), response to stress (125 proteins, blue), regulation of cell death (74 proteins, amber), and apoptosis (28 proteins, brown). 
bioRxiv preprint doi: https://doi.org/10.1101/2021.07.30.454526; this version posted August 4, 2021. The copyright holder for this preprint (which was not certified by peer review) is the author/funder, who has granted bioRxiv a license to display the preprint in perpetuity. It is made available under aCC-BY 4.0 International license.

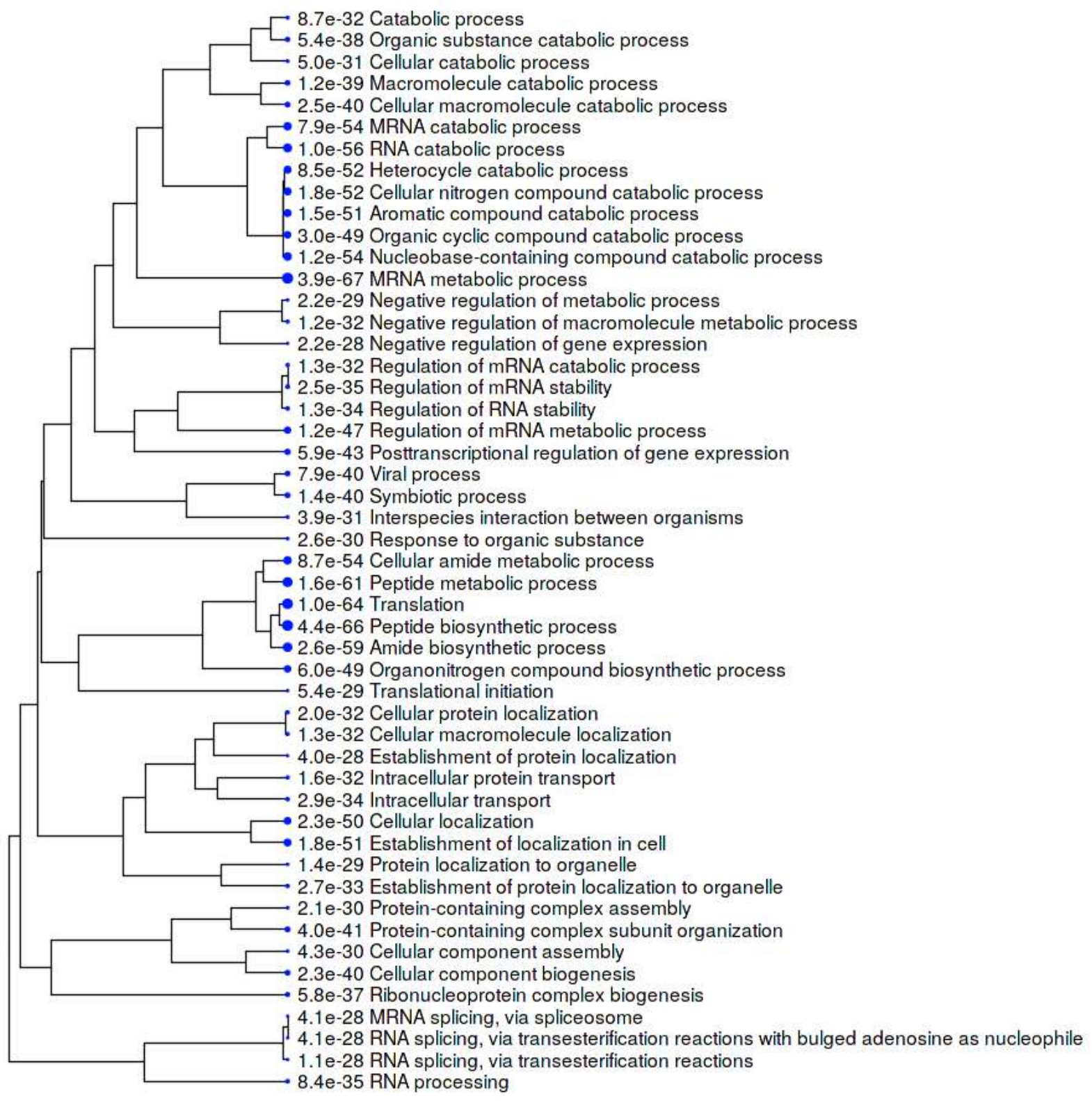

Fig. 4. Hierarchical clustering of the top $50 \mathrm{GO}$ Biological Processes associated with the master autoantigen-ome of 751 DS-affinity autoAgs. Bigger blue dots indicate more significant $p$ values. 
bioRxiv preprint doi: https://doi.org/10.1101/2021.07.30.454526; this version posted August 4, 2021. The copyright holder for this preprint (which was not certified by peer review) is the author/funder, who has granted bioRxiv a license to display the preprint in perpetuity. It is made available under aCC-BY 4.0 International license.

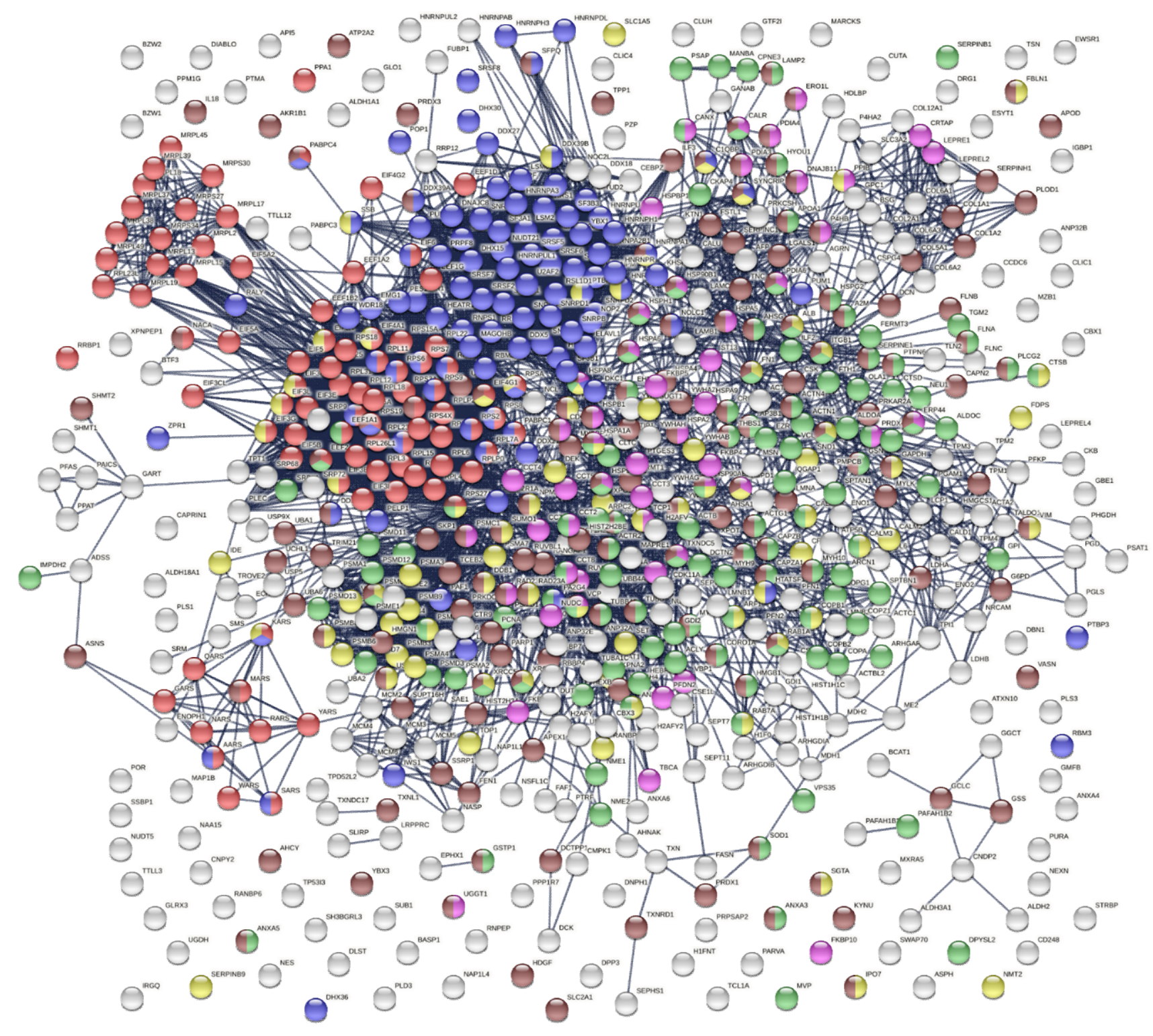

Fig. 5. The COVID autoantigen-ome of 657 autoAg candidates. Lines represent protein-protein interactions with highest confident level. Colored proteins are associated with translation (87 proteins, red), RNA processing (103 proteins, blue), protein folding (51 proteins, pink), symbiont process (78 proteins, yellow), vesicle-mediated transport (125 proteins, green), and response to stress (161 proteins, brown). 
bioRxiv preprint doi: https://doi org/10.1101/2021.07.30.454526; this version posted August 4, 2021. The copyright holder for this preprint (which was not certified by peer review) is the author/funder, who has granted bioRxiv a license to display the preprint in perpetuity. It is made available under aCC-BY 4.0 International license.
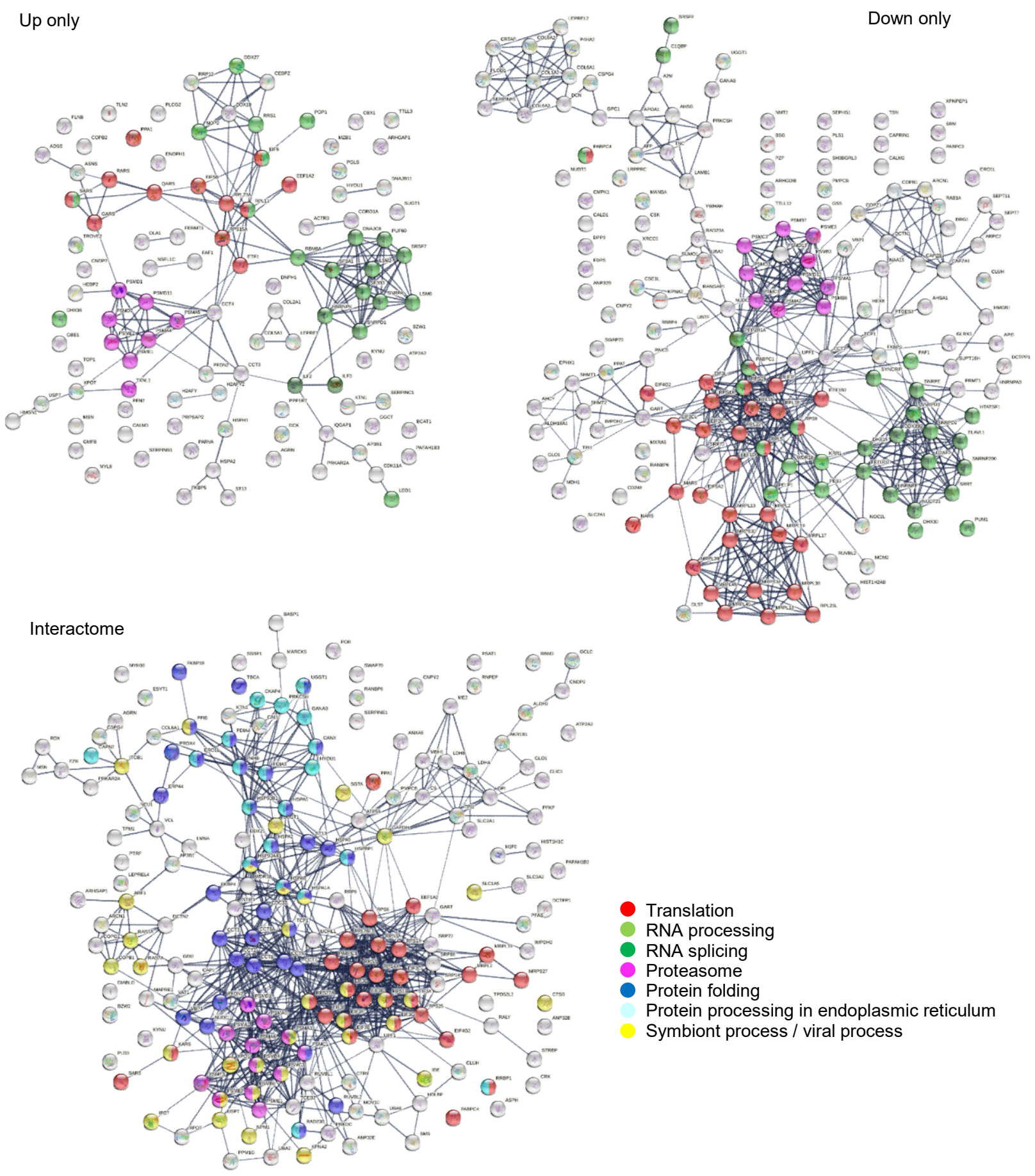

Translation

RNA processing

RNA splicing

Proteasome

Protein folding

Protein processing in endoplasmic reticulum

Symbiont process / viral process

Fig. 6. COVID-affected autoAgs that are found up-regulated only, down-regulated only, or interacting with SARS-Cov-2 proteins. Note the significant enrichment of proteins associated with translation, RNA processing and splicing, and other processes. 

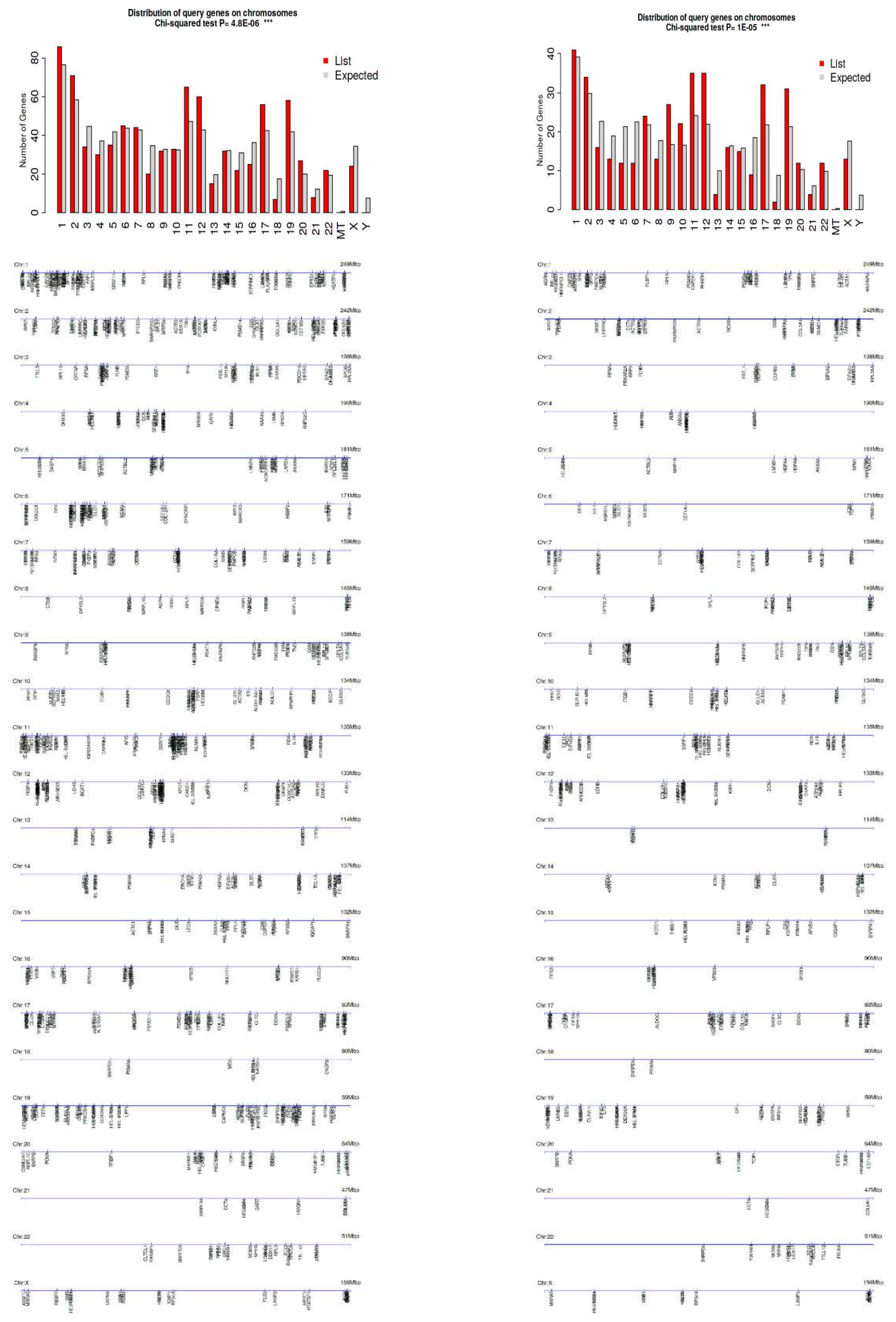

Fig. 7. Distribution of autoAg coding genes by chromosomes. Left: 751 confirmed and putative autoAgs. Right: 400 confirmed autoAgs only. 
A
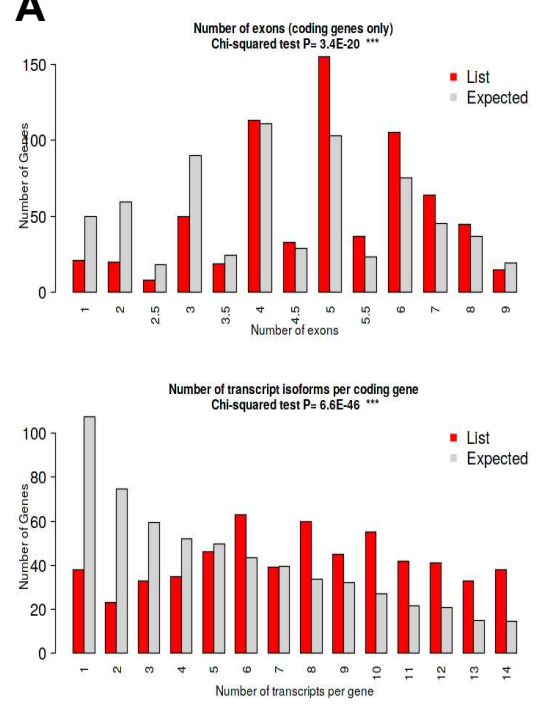

B
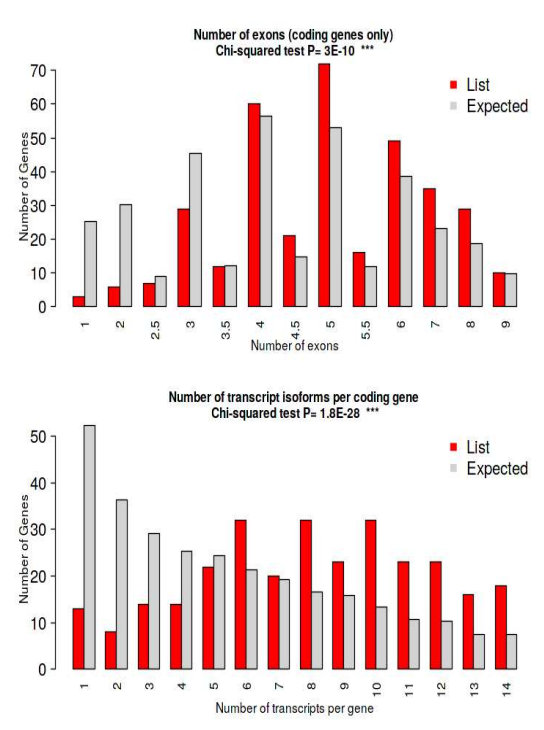
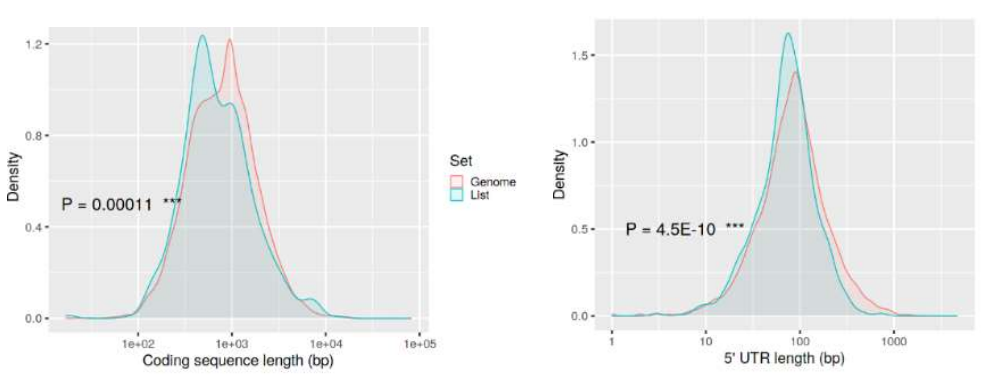

Set
$\begin{aligned} & \text { Genorre } \\ & \text { List }\end{aligned}$
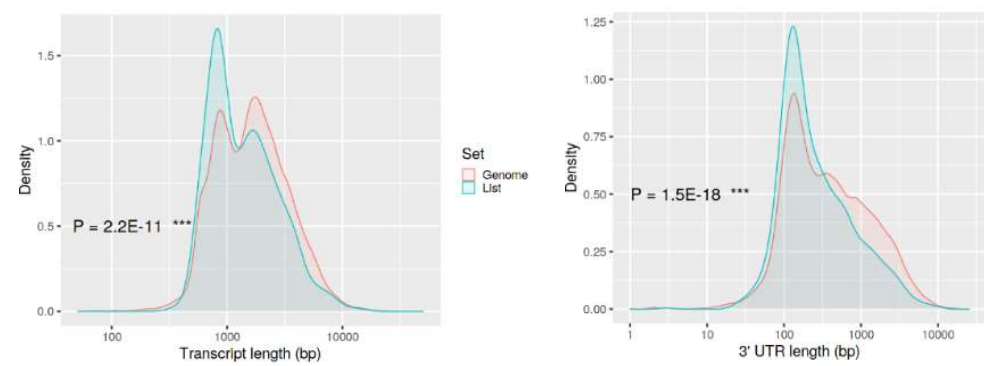

Set
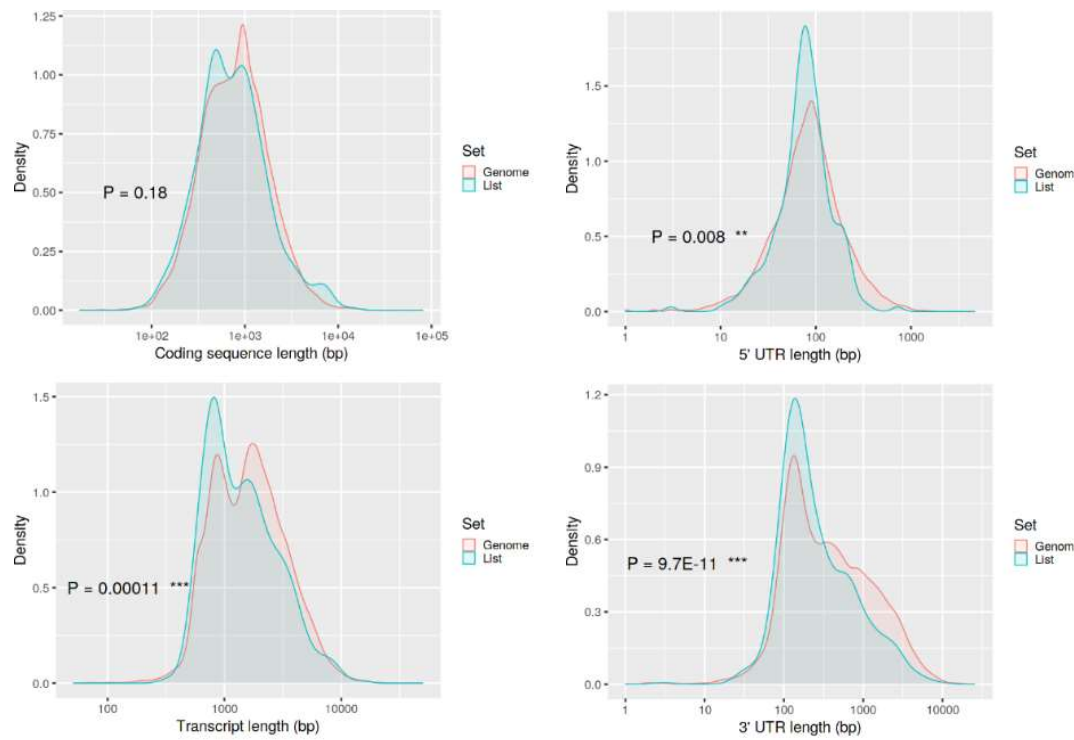

Fig. 8. Characteristics of the autoAg coding genes compared with the rest in the genome. Differences are evaluated with Chi-squred and Student's t-tests. (A) 751 confirmed and putative autoAgs. (B) 400 confirmed autoAgs. 


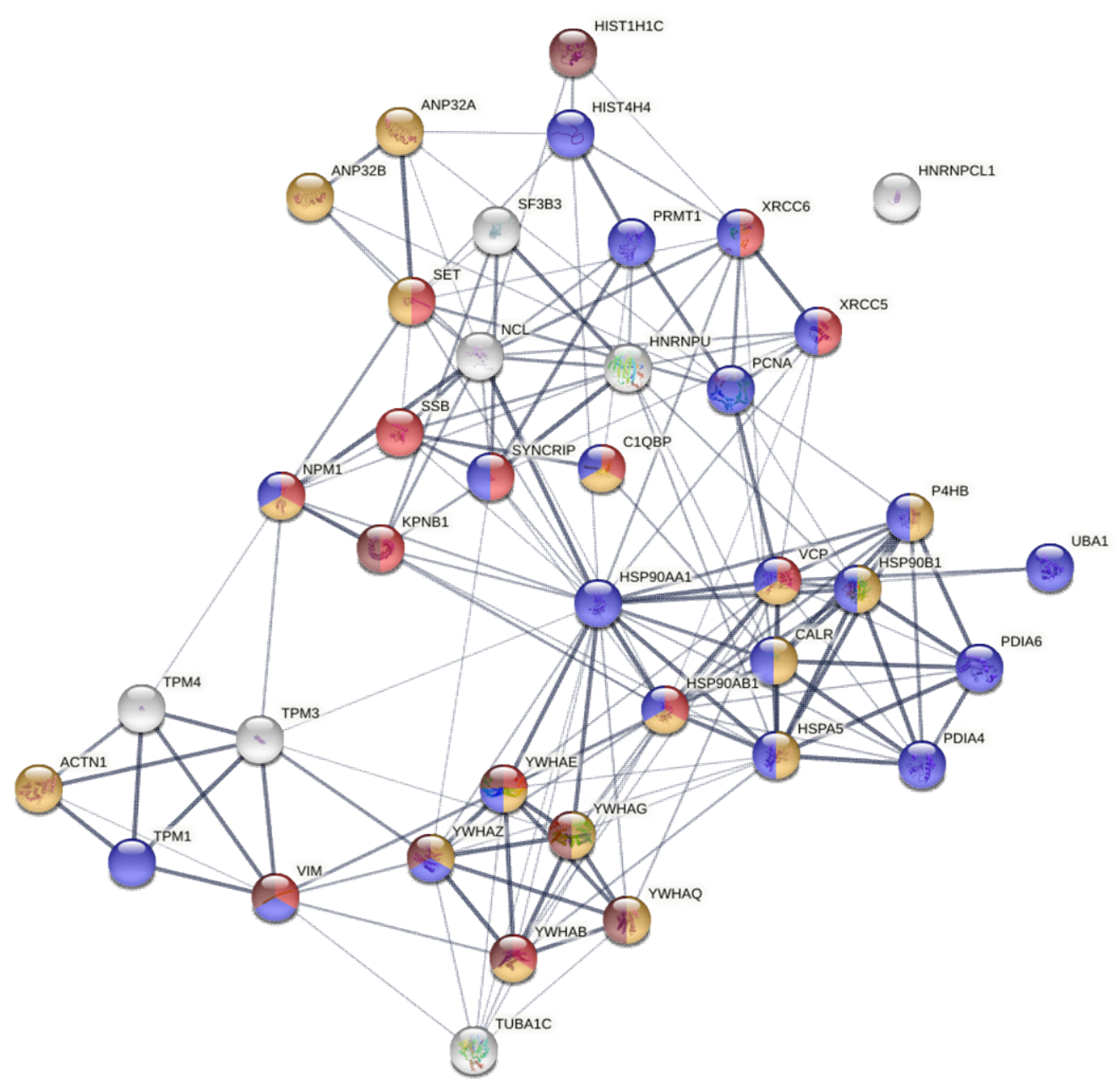

Fig. 9. Common autoAgs identified from all six cell types examined in this study. Colored are proteins associated with viral infection (13 proteins, red), regulation of apoptotic process (17 proteins, amber), response to stress (22 proteins, blue), and apoptosis ( 8 proteins, brown). 
bioRxiv preprint doi: https://doi.org/10.1101/2021.07.30.454526; this version posted August 4, 2021. The copyright holder for this preprint (which was not certified by peer review) is the author/funder, who has granted bioRxiv a license to display the preprint in perpetuity. It is made available under aCC-BY 4.0 International license.
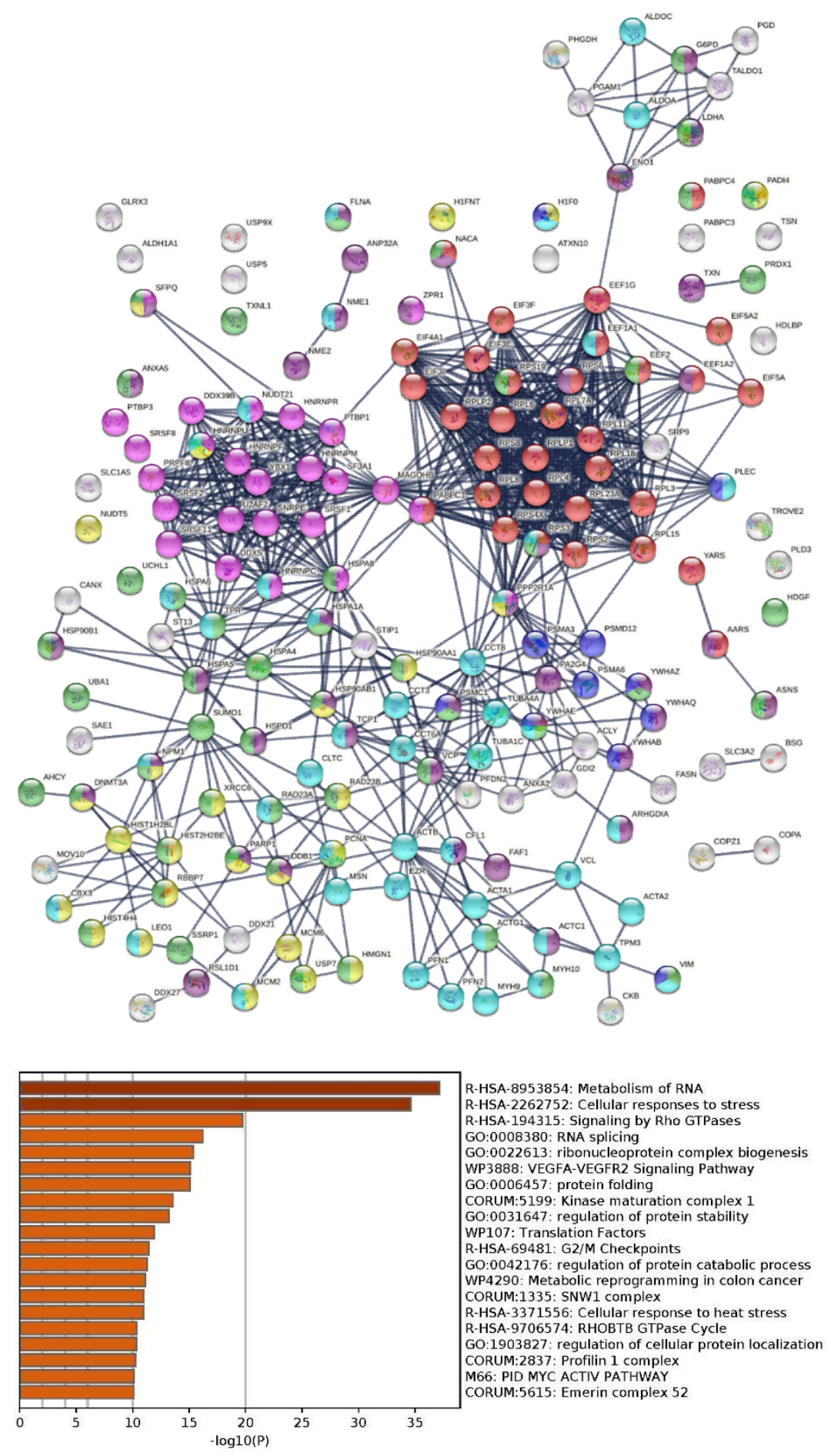

Fig. 10. Top: Potential autoAgs affected by ubiquitination in SARS-Cov-2 infection (lines represent protein-protein interactions with the highest confidence). Colored are proteins associated with translation (32 proteins, red), RNA splicing (25 proteins, pink), regulation of cell death (40 proteins, dark purple), chromosome organization (26 proteins, yellow), response to stress (50 proteins, green), cytoskeleton (45 proteins, aqua), and apoptosis (11 proteins, blue). Bottom: Top 20 enriched processes and pathways associated with the ubiquitinated autoAgs . 